\title{
Formation of the regular satellites of giant planets in an extended gaseous nebula I: subnebula model and accretion of satellites
}

\author{
Ignacio Mosqueira $^{\mathrm{a}, *}$ and Paul R. Estrada ${ }^{\mathrm{b}}$ \\ ${ }^{\text {a }}$ NASA Ames/SETI Institute, Mail Stop 245-3, Moffett Field, CA 94305, USA \\ ${ }^{\mathrm{b}}$ Astronomy Department, 406 Space Sciences Building, Cornell University, Ithaca, NY 14850, USA
}

Received 14 November 2001; revised 27 January 2003

\begin{abstract}
We model the subnebulae of Jupiter and Saturn wherein satellite accretion took place. We expect each giant planet subnebula to be composed of an optically thick (given gaseous opacity) inner region inside of the planet's centrifugal radius (where the specific angular momentum of the collapsing giant planet gaseous envelope achieves centrifugal balance, located at $r_{C}^{J} \sim 15 R_{\mathrm{J}}$ for Jupiter and $r_{C}^{S} \sim 22 R_{\mathrm{S}}$ for Saturn) and an optically thin, extended outer disk out to a fraction of the planet's Roche-lobe $\left(R_{H}\right)$, which we choose to be $\sim R_{H} / 5$ (located at $\sim 150 R_{\mathrm{J}}$ near the inner irregular satellites for Jupiter, and $\sim 200 R_{\mathrm{S}}$ near Phoebe for Saturn). This places Titan and Ganymede in the inner disk, Callisto and Iapetus in the outer disk, and Hyperion in the transition region. The inner disk is the leftover of the gas accreted by the protoplanet. The outer disk may result from the nebula gas flowing into the protoplanet during the time of giant planet gap-opening (or cessation of gas accretion). For the sake of specificity, we use a solar composition "minimum mass" model to constrain the gas densities of the inner and outer disks of Jupiter and Saturn (and also Uranus). Our model has Ganymede at a subnebula temperature of $\sim 250 \mathrm{~K}$ and Titan at $\sim 100 \mathrm{~K}$. The outer disks of Jupiter and Saturn have constant temperatures of 130 and $90 \mathrm{~K}$, respectively.

Our model has Callisto forming in a time scale $\sim 10^{6}$ years, Iapetus in $10^{6}-10^{7}$ years, Ganymede in $10^{3}-10^{4}$ years, and Titan in $10^{4}-10^{5}$ years. Callisto takes much longer to form than Ganymede because it draws materials from the extended, low density portion of the disk; its accretion time scale is set by the inward drift times of satellitesimals with sizes $300-500 \mathrm{~km}$ from distances $\sim 100 R_{\mathrm{J}}$. This accretion history may be consistent with a partially differentiated Callisto with a $\sim 300-\mathrm{km}$ clean ice outer shell overlying a mixed ice and rock-metal interior as suggested by Anderson et al. (2001), which may explain the Ganymede-Callisto dichotomy without resorting to fine-tuning poorly known model parameters. It is also possible that particulate matter coupled to the high specific angular momentum gas flowing through the gap after giant planet gap-opening, capture of heliocentric planetesimals by the extended gas disk, or ablation of planetesimals passing through the disk contributes to the solid content of the disk and lengthens the time scale for Callisto's formation. Furthermore, this model has Hyperion forming just outside Saturn's centrifugal radius, captured into resonance by proto-Titan in the presence of a strong gas density gradient as proposed by Lee and Peale (2000). While Titan may have taken significantly longer to form than Ganymede, it still formed fast enough that we would expect it to be fully differentiated. In this sense, it is more like Ganymede than like Callisto (Saturn's analog of Callisto, we expect, is Iapetus). An alternative starved disk model whose satellite accretion time scale for all the regular satellites is set by the feeding of planetesimals or gas from the planet's Roche-lobe after gap-opening is likely to imply a long accretion time scale for Titan with small quantities of $\mathrm{NH}_{3}$ present, leading to a partially differentiated (Callisto-like) Titan. The Cassini mission may resolve this issue conclusively. We briefly discuss the retention of elements more volatile than $\mathrm{H}_{2} \mathrm{O}$ as well as other issues that may help to test our model.
\end{abstract}

(C) 2003 Elsevier Science (USA). All rights reserved.

Keywords: Galilean satellites; Gas dynamics; Formation of satellites; Protoplanetary disks

\section{Introduction}

The regular satellites of Jupiter and Saturn generally have low inclinations and eccentricities. Perhaps most strik-

\footnotetext{
* Corresponding author. Fax: +1-650-604-6779.

E-mail address: mosqueir@cosmic.arc.nasa.gov (I. Mosqueira).
}

ing is the progression of satellite density in the Galilean system. Also, the ratios between the satellite systems and the parent bodies of mass and angular momentum are quite similar (Pollack et al., 1991), which suggests a common origin in an accretion disk present about the protoplanets at a late stage of their formation. These properties, taken together with the tantalizing ratio of the largest satellite of 
each system to its primary $M_{s} / M_{P} \sim 10^{-4}$ (not too dissimilar from the ratio of giant planet to Sun), lead one to think of the Galilean satellite system as a kind of scaled-down solar system.

Given their similarities in distances, masses, and densities, an issue we wish to focus on is how to view Titan in light of the Galilean satellite system, especially Ganymede and Callisto. Yet, the differences between these three satellites are as intriguing as their similarities. The Galileo mission moment of inertia data are consistent with a fully differentiated Ganymede, but only a partially differentiated Callisto (Anderson et al., 1998). Moreover, Callisto shows no evidence of tectonic activity. Also, the association of craters with the presence of $\mathrm{CO}_{2}$ in Callisto but not Ganymede (Hibbitts et al., 2000) as well as the degradation of craters presumably due to the sublimation of $\mathrm{CO}_{2}$ in Callisto but not Ganymede (Moore et al., 1999), which is consistent with the presence of a $\mathrm{CO}_{2}$ atmosphere in Callisto (Carlson, 1999), seems to require that Callisto be assembled with and retain oxidized ices more volatile than $\mathrm{H}_{2} \mathrm{O}$. In the case of Titan, it is probably the presence of methane in the atmosphere that has received the most attention (Lunine et al., 1989; Prinn and Fegley, 1989).

Recently, Anderson et al. (2001) have investigated two and three layer models for Callisto's internal structure assuming hydrostatic equilibrium. For the two layer models these authors find two limiting cases: a relatively pure ice shell about $\sim 300 \mathrm{~km}$ overlying a mixed ice and rock-metal interior, and a thick $\gtrsim 1000-\mathrm{km}$ ice and rock-metal outer shell overlying a rock-metal core. Since it is difficult to reconcile a metallic core with a partially differentiated state the former solution appears more likely. Given that accreting bodies allocate a fraction of their energy as surface heat (Schubert et al., 1981; Coradini et al., 1982), fast satellite accretion would melt the water ice and lead to rock separation and runaway differentiation (Friedson and Stevenson, 1983). Previous attempts to explain an undifferentiated Callisto have relied on fine-tuning parameters (Schubert et al., 1981; Coradini et al., 1982; Lunine and Stevenson, 1982). Although it is possible that nonhydrostatic effects in Callisto's core could be large enough to allow for complete differentiation of this satellite and still be sufficiently small in Ganymede's core to have avoided detection, we regard this possibility as unlikely. Instead, we favor a model that makes Callisto slowly.

Other issues also seem difficult to explain. For instance, one might expect the outermost Galilean satellite to have significantly less angular momentum than the preceding satellite. It would seem unlikely that the satellite disk would have enough surface density to make a satellite the size of Callisto at $26 R_{\mathrm{J}}$, but form no smaller objects outside its orbit. Furthermore, the separation between Ganymede and Callisto $\left(\sim 10 R_{\mathrm{J}}\right)$ is so large that one is led to wonder why there are no satellites in between at $\sim 20 R_{\mathrm{J}}$ (see Mosqueira and Estrada (2003), hereafter Paper II, for a brief discussion of orbital stability). One can always argue serendipity, but the Galilean satellite system is sufficiently regular that we reserve this explanation as a last resort.

A related point can be made concerning Titan and Iapetus. If we form the satellites out of a continuous, smoothly varying accretion disk, it would seem difficult to explain why there are no large satellites between Titan at $\sim 20 R_{\mathrm{S}}$ and Iapetus at $\sim 60 R_{\mathrm{S}}$ (Hyperion does not have enough mass to affect this argument).

Also, one must account for the differences between the satellite systems of Jupiter and Saturn. In the case of Saturn's satellite system, the concentration of mass in Titan needs to be addressed. But perhaps the most perverse difference between the two satellite systems is the fact that whereas the Galilean satellites get rockier closer to the planet, the inner satellites of Saturn appear to be made mostly of ice! Even so, we attempt a combined model for both Jupiter and Saturn (as well as Uranus).

If we take the satellite systems of Jupiter and Saturn and add the amount of gas necessary to create a solar composition mixture the resulting disks have a total angular momentum comparable to the spin angular momentum of the parent planet (Stevenson et al., 1986). The issue arises whether or not one would expect the circumplanetary disk to exhibit a solar mixture of elemental abundances of water and ice bearing materials. One can think of several processes that modified the abundances of rock and ice from their solar abundances. Yet, the fact that the similarly sized Ganymede, Callisto, and Titan all deviate from solar mixture by the same proportion $(\sim 60 \%$ rock, $\sim 40 \%$ ice by mass) seems to indicate that one should be guided by solar mixtures and investigate mechanisms for deviation from them, such as size-dependent water vaporization on one end and water enrichment by composition selective mechanisms on the other. If so, one might calculate models with "minimum mass" by augmenting the mass of the satellites by some factor (typically $\sim 100$; Pollack et al., 1994), corresponding to the mass ratio of gas to rock-metal/ice in the solar nebula. This factor might be decreased somewhat in view of the heavy-element enrichment of the giant planets or increased in view of the possible loss of some of the accreting materials as a result of the specifics of the process used to make the planet and satellites.

In order to arrive at a specific model for the formation of regular satellites in a gaseous medium we need to characterize the subnebular viscosity. It has been suggested that because of the stabilizing influence of a positive radial gradient in specific angular momentum, turbulence in a Keplerian disk is not self-sustaining unless a source of "stirring" is found (Ryu and Goodman, 1992; Balbus, Hawley, and Stone, 1996). As a result, one needs to identify a specific mechanism that can maintain turbulence in the dense, high orbital frequency subnebula. One such suggestion is that convection drives turbulence (Cameron, 1978a; Lin and Papaloizou, 1980; Ruden and Lin, 1986); however, eventually particle growth may stop convection by diminishing the Rosseland mean opacity and weakening its tem- 
perature dependence (Weidenschilling and Cuzzi 1993). Given the fast dynamical time scale and the high particle density of the subnebula disk, coagulation and settling for sticky particles may take place on a time scale faster than disk evolution. Furthermore, if convection drives turbulence then angular momentum transport may be weak $\left(\alpha \sim 10^{-5}\right.$; Stone and Balbus, 1996) and directed inwards (Ryu and Goodman, 1992; Kley et al., 1993; Stone and Balbus, 1996; Cabot, 1996), which would essentially terminate gas accretion onto the primary. Another possibility is that turbulence is driven by a magnetohydrodynamic (MHD) instability (Balbus and Hawley, 1991). But this is also unlikely to apply (Gammie, 1996) in the dense and relatively cool subnebula disk. Alternatively, there are a variety of ways that accretion itself, or the gravitational energy released by it, can provide the source of free energy that can drive turbulence. It has been pointed out (but not quantitatively explored) that a turbulent shear layer, where the angular momentum of the infalling gas is adjusted to the angular momentum of the Keplerian disk flow, exists below an accretion shock and may provide a localized viscosity (Cassen and Moosman, 1981; Cassen and Summers, 1983). More recently it has been shown that a bump in the temperature profile of the disk, as may result from accretion, that leads to a strong radial entropy gradient generates Rossby waves and localized turbulence (Lovelace et al., 1999; Li et al., 2000). Similarly, but more generally, Klahr and Bodenheimer (2001) study a global baroclinic instability as a source of turbulence and outward angular momentum transport in Keplerian accretion disks characterized by a negative radial entropy gradient.

To create a coherent scenario of satellite formation, the source of the solids that go into the satellite systems must be considered. The concentration of rock/ice to gas in the subnebula may depend on the ability of the protoplanet to disturb the orbits of planetesimals situated within a few AU of its orbit into ones that crossed its orbit. One would expect that in a time scale much shorter than the lifetime of the solar system virtually all the planetesimals located in the outer solar system would have their orbits perturbed into giant planet crossing orbits (Gladman and Duncan, 1990). What happens to such a planetesimal depends on the size of the planet at the time of crossing. If the giant planet's envelope filled a fair fraction of its Hill radius, as it probably did during most or all of its gas accretion phase (unless significant amounts of gas accreted through the gap after gap-opening), then the distended atmosphere would have greatly increased the planet's cross-section (Bodenheimer and Pollack, 1986; Pollack et al., 1996). Early arriving (before the completion of planetary accretion) icy planetesimals of size $<10 \mathrm{~km}$ (Zahnle, private communication) may break up in the contracting envelope of the giant planet, and their condensable content may then be entrapped in the gas and left behind in the form of a circumplanetary disk. On the other hand, most late arriving planetesimals may have been scattered to further regions of the solar system with some sent to the Oort cloud and some lost altogether. Thus, our model relies on early arriving planetesimals that break up or dissolve in the extended giant-planet envelopes to provide the bulk of the material that will eventually make the satellite systems, delivered to the satellite disk in a time scale given by the envelope collapse time.

This formation model is consistent both with a model that captures irregular satellites at a time when the protoplanetary envelope was collapsing rapidly and extended several hundred planetary radii (Pollack et al., 1979) and with a model that captures irregular satellites using a longlived circumplanetary gas disk (Cuk and Burns, 2002). Here late arriving interplanetary debris plays a role in that it can threaten the survival of regular satellites close to their primary. Hence, the large disparity in masses between Titan and all other moons of Saturn may in part be the result of the break-up of satellites by high-velocity impacts (e.g., Lissauer, 1995; but note that gas would still be needed to clear up the collisional debris and prevent re-accretion). In contrast, a starved disk model (Stevenson, 2001) relies on the late arriving planetesimals or flow through the gap to form a disk around the planet out of which all the regular satellites will eventually accrete. One should keep in mind, however, that most planetesimals were probably scattered or the giant planets would have ended up with too much high-Z mass (Podolak et al., 1993) and that most of the mass in the nebula disk at late times is in the form of planetesimals (Mizuno et al., 1978; Weidenschilling, 1997). Furthermore, the high specific angular momentum of gas arriving at late times may place it in orbit well outside the region where most of the satellite mass is found.

In Section 2 we organize the satellite systems of the giant planets according to the Hill radius of the primary. In Section 3 we characterize the subnebulae of giant planets, especially that of Jupiter. In Section 4 we discuss the accretion of the Galilean satellites, reserving discussion of Callisto for Section 5. In Section 6 we turn to Saturn's satellite system. In Section 7 we discuss the satellite system of Uranus. In Section 8 we make some comments on an alternative satellite accretion model that leads to a long accretion time scale for every satellite. In Section 9 we present our conclusions and discussion. In Paper II we turn to the migration and survival of full-sized satellites.

\section{Regular satellites of giant planets}

We begin with a brief comparative discussion of the satellite systems of the giant planets. We compare satellite positions mainly in terms of the Hill radius $R_{\mathrm{H}}=$ $a\left(M_{P} / 3 M_{\odot}\right)^{1 / 3}$ of the planet (and the concomitant centrifugal radius $r_{\mathrm{c}} \approx R_{\mathrm{H}} / 48$; see Sect. 3 ). In Fig. 1, we plot the locations of the regular satellites (solid circles) and the innermost irregular satellites (open circles) in units of the Hill radius of the giant planet. The bold dashed line describes the position of the centrifugal radius. From this 

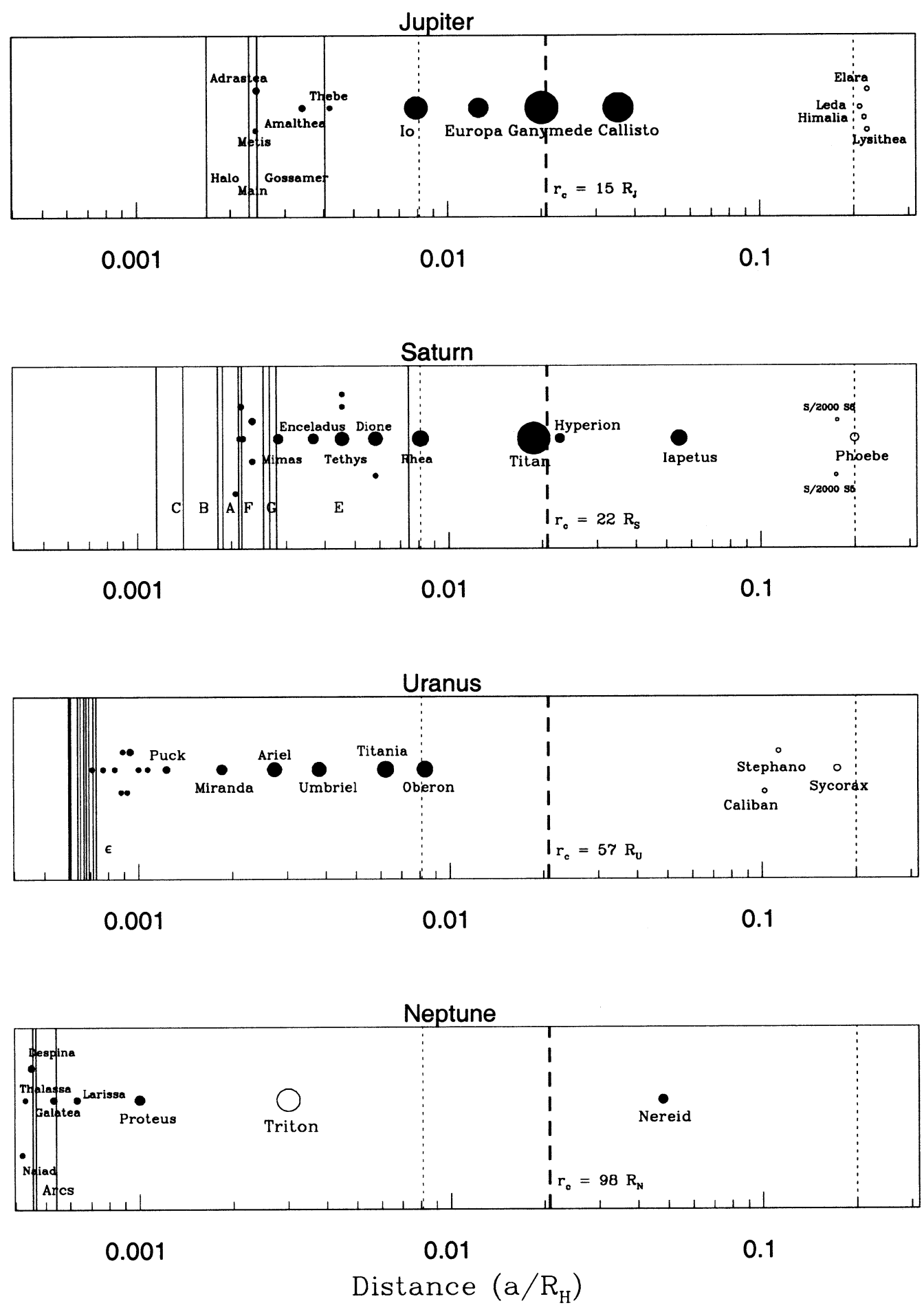

117

Fig. 1. Comparison of the jovian, saturnian, and uranian satellite systems with the distance scale in terms of the respective planet's Hill radius $\left(R_{\mathrm{H}}^{\mathrm{J}} \sim 750 R_{\mathrm{J}}\right.$, $R_{\mathrm{H}}^{\mathrm{S}} \sim 1100 R_{\mathrm{S}}, R_{\mathrm{H}}^{\mathrm{U}} \sim 2740 R_{\mathrm{U}}$ ). Planetary rings are denoted by solid lines and are labeled when possible. The centrifugal radius is denoted by a bold dashed line. Dotted lines correspond to positions of interest. The first, located at the distance of Rhea for Saturn, corresponds to the inner portion of the disk. The second corresponds to the outer edge of the disk $\left(\sim R_{\mathrm{H}} / 5\right)$ in our models.

plot it is immediately apparent that the irregulars (presumed to be captured objects) of the three inner giant planets are far from the location of the centrifugal radius. We expect that this observation means that the gas disk which gave rise to the regular satellite systems extended well outside this radius (out to $\sim R_{\mathrm{H}} / 5$ for Jupiter and Saturn). Jupiter and Saturn have regular satellites which are far outside the centrifugal radius, with Saturn's Iape- 
Table 1

Satellite data ${ }^{\mathrm{a}}$

\begin{tabular}{|c|c|c|c|c|c|}
\hline & Distance $\left(R_{P}\right)$ & Radius (km) & Density $\left(\mathrm{g} \mathrm{cm}^{-3}\right)$ & Mass $\left(10^{26} \mathrm{~g}\right)$ & $\mu^{\mathrm{d}}\left(10^{-4}\right)$ \\
\hline Jupiter & & 71492 & 1.326 & 18980 & 9.5 \\
\hline Io & 5.905 & 1,821 & 3.53 & 0.894 & 0.47 \\
\hline Europa & 9.937 & 1,565 & 2.97 & 0.480 & 0.25 \\
\hline Ganymede & 14.99 & 2,634 & 1.94 & 1.4823 & 0.78 \\
\hline Callisto & 26.37 & 2,403 & 1.85 & 1.0776 & 0.57 \\
\hline Leda $^{c}$ & 155.2 & 5 & $?$ & $?$ & $?$ \\
\hline Himalia $^{\mathrm{c}}$ & 160.6 & 85 & $?$ & $?$ & $?$ \\
\hline Lysithea $^{\mathrm{c}}$ & 163.9 & 12 & $?$ & $?$ & $?$ \\
\hline Elara $^{\mathrm{c}}$ & 164.2 & 40 & $?$ & $?$ & $?$ \\
\hline Saturn & & 60330 & 0.687 & 5684.6 & 2.9 \\
\hline Mimas & 3.075 & 199 & 1.12 & $3.7 \times 10^{-4}$ & $6.5 \times 10^{-4}$ \\
\hline Enceladus & 3.945 & 249 & 1.00 & $6.5 \times 10^{-4}$ & $1.1 \times 10^{-3}$ \\
\hline Tethys & 4.884 & 529 & 0.98 & $6.1 \times 10^{-3}$ & 0.010 \\
\hline Dione & 6.256 & 560 & 1.49 & 0.011 & 0.019 \\
\hline Rhea & 8.736 & 764 & 1.24 & 0.023 & 0.040 \\
\hline Titan & 20.25 & 2,575 & 1.88 & 1.3457 & 2.4 \\
\hline Hyperion & 24.55 & $185 \times 113$ & $?$ & $?$ & $?$ \\
\hline Iapetus & 59.03 & 720 & 1.0 & 0.016 & 0.028 \\
\hline $\mathrm{S} / 2000 \mathrm{~S} 5^{\mathrm{b}, \mathrm{c}}$ & 187.3 & 10 & $?$ & $?$ & $?$ \\
\hline $\mathrm{S} / 2000 \mathrm{~S} 6^{\mathrm{b}, \mathrm{c}}$ & 189 & 16 & $?$ & $?$ & $?$ \\
\hline Phoebe $^{c}$ & 214.5 & $115 \times 105$ & $?$ & $?$ & $?$ \\
\hline Uranus & & 25559 & 1.318 & 868.32 & 0.44 \\
\hline Puck & 3.36 & 77 & $?$ & $?$ & $?$ \\
\hline Miranda & 5.08 & $240 \times 233$ & 1.20 & $6.59 \times 10^{-4}$ & $7.6 \times 10^{-3}$ \\
\hline Ariel & 7.48 & $581 \times 578$ & 1.67 & 0.0135 & 0.16 \\
\hline Umbriel & 10.4 & 585 & 1.4 & 0.0117 & 0.13 \\
\hline Titania & 17.05 & 790 & 1.71 & 0.0353 & 0.41 \\
\hline Oberon & 22.8 & 760 & 1.63 & 0.0301 & 0.35 \\
\hline Caliban $^{\mathrm{c}}$ & 280.5 & 30 & $?$ & $?$ & $?$ \\
\hline Stephano ${ }^{\text {b,c }}$ & 309 & 10 & $?$ & $?$ & $?$ \\
\hline Sycorax $^{c}$ & 477.9 & 60 & $?$ & $?$ & $?$ \\
\hline Neptune & & 24766 & 1.638 & 1024.3 & 0.51 \\
\hline Proteus & 4.75 & $218 \times 201$ & $?$ & $?$ & $?$ \\
\hline Triton $^{\mathrm{c}}$ & 14.32 & 1,353 & 2.05 & 0.215 & 2.1 \\
\hline Nereid & 222.6 & 170 & $?$ & $?$ & $?$ \\
\hline
\end{tabular}

${ }^{a}$ From Beatty (1999).

${ }^{\mathrm{b}} \mathrm{P}$. Nicholson, private communication.

${ }^{\mathrm{c}}$ Irregular satellite.

${ }^{\mathrm{d}}$ Mass of secondary over mass of primary.

tus located much farther out than Jupiter's Callisto. These two planets also have satellites just inside (Saturn also just outside) the centrifugal radius. In contrast, one has to go to $\left(\sim R_{\mathrm{H}} / 100\right)$ to find any regular uranian satellites.

We add the satellite system of Neptune for the sake of completeness; however, Goldreich et al. (1989) (see also McKinnon, 1984) showed that retrograde Triton is likely to be a captured object. Since these authors estimate a collision probability near one between $\sim 5 R_{\mathrm{N}}$ and the centrifugal radius $\sim 100 R_{\mathrm{N}}$, Triton's capture would have broken up or scattered any pre-existing satellites in this region. Nereid's high eccentricity and inclination, and large semi-major axis are best understood in terms of this process. Hence, we will not discuss this satellite system here, though we expect that prior to Triton's capture it may have been analogous to that of Uranus.

In Table I we provide data on both regular and irregular satellites for all four giant planets. Because small satellites close to the planet are likely to have undergone significant collisional evolution since the time of their formation, we exclude them. The formation history of coorbitals remains to be investigated, and we shall not do so here; hence, coorbitals have also been excluded from the table.

\section{The giant planet subnebula}

The "minimum" mass subnebula we use here is one of solar nebula composition that provides just enough mass to form the observed satellite systems with the observed rock/ ice mass ratio. Given Jupiter's relative enrichment in heavy elements with respect to the solar nebula, the minimum mass subnebula is not a firm lower bound. On the other hand, inefficiencies in the satellite formation process and depletion of solids due to planetesimal formation mean that it is not a firm upper bound either. Still, it remains a useful 
reference and we have chosen it for the sake of specificity. As we will see, it is possible that a minimum mass subnebula does in fact apply. If anything, we expect that the concentration of solids will turn out to be larger than solar proportion. Here and in Paper II, we will be guided by solar proportion, but we will also consider solid enhancement factors of 3-4 in rough agreement with giant planet high-Z enhancement.

Gas flowing into the Hill sphere will form a high optical depth hydrostatic gas disk around the protoplanet. We can obtain an estimate of the size of this disk by assuming that infalling gas elements conserve specific angular momentum. We reproduce here an estimate of the specific angular momentum flowing into the Hill sphere of the planet for a case such that the planet travels on a circular trajectory and the gas moves on Keplerian orbits (gas pressure support tends to decrease the specific angular momentum flowing into the planet, but this is probably a small correction). Assuming that the giant planet atmosphere fills its lobe, the specific angular momentum received by the envelope is approximately given by (Lissauer, 1995)

$$
\ell \approx-\Omega \frac{\int_{0}^{R_{\mathrm{H}}} \frac{3}{2} x^{3} d x}{\int_{0}^{R_{\mathrm{H}}} x d x}+\Omega R_{\mathrm{H}}^{2} \approx \frac{1}{4} \Omega R_{\mathrm{H}}^{2},
$$

where $\Omega=\left(G M_{\odot} / a^{3}\right)^{1 / 2}, a$ is the planet's semimajor axis, and $R_{\mathrm{H}}$ is the planet's Hill radius. The specific angular momentum given above has two contributions. The first is due to the angular momentum flux flowing into the planet's Hill radius (over those portions where the mass flux is inward) as a result of the Keplerian shear divided by the mass flux in the rotating frame. The second contribution is a correction to translate back to an inertial frame (see Lissauer, 1995, and references therein). This expression neglects the gravitational effect of the planet and assumes that the angular momentum of the infalling gas is absorbed by the giant planet's extended envelope, which then sets the angular momentum for the resulting accretion disk. We note that the giant planet accretes gas from locations in the disk well outside $R_{\mathrm{H}}$. This calculation assumes that prior to gap-opening the angular momentum flux is dominated by gas elements with semi-major axes originating from $\sim R_{\mathrm{H}}$ of the proto-planet. Using the above estimate and equating the centrifugal to gravitational forces $\ell^{2} / r_{c}^{3} \approx G M_{P} / r_{c}^{2}$, where $M_{P}$ is the planetary mass, one obtains the centrifugal radius $r_{c} \approx R_{\mathrm{H}} / 48$ (Cassen and Pettibone, 1976; Stevenson et al., 1986). For Jupiter and Saturn these radii are located at $r_{c}^{J} \approx$ $15 R_{\mathrm{J}}$ and $r_{c}^{S} \approx 22 R_{\mathrm{S}}$, close to the positions of Ganymede and Titan, respectively.

It is tempting to conclude that this is roughly the size of the gas disk that led to the formation of the satellites. One must keep in mind, however, that Callisto is at $26 R_{\mathrm{J}}$ nearly twice the size of Jupiter's centrifugal radius, and Iapetus is at $59 R_{\mathrm{S}}$ nearly three times the size of Saturn's centrifugal radius. Our model accounts for these facts by using a two component subnebula. The extent of the outer disk is difficult to pin down. One may try to estimate the specific angular momentum of the gas flowing through the gap after gap-opening, as seen in some simulations (Artymowicz and Lubow, 1996) where accretion continues through the planetary Lagrange points. In this case we cannot ignore the gravitational effect of the planet, since doing so would result in no accretion at all, i.e., after gap-opening gas elements would not cross the sphere of influence of the planet. Instead, we try to estimate the specific angular momentum of the gas as it passes through the Lagrange points. Assuming that the inflow takes place at a low velocity in the rotating frame and it is directed nearly toward the planet, we can obtain an estimate to the specific angular momentum of the gas flowing through the gap by keeping only the change of frame contribution of Eq. (1) and get $\ell \sim \Omega R_{\mathrm{H}}^{2}$. If we now assume conservation of angular momentum, the radius at which this component would achieve centrifugal balance is given by $\sim R_{\mathrm{H}} / 3$, as suggested by Quillen and Trilling (1998). However, how much gas flows through the gap depends on the assumed turbulence of the nebula.

For now we assume that Callisto derived its condensables from the materials present in the outer disk, and we justify this assumption later in this paper (where we show that inwardly migrating satellitesimals are effectively captured by satellite embryos with sizes $\sim 10^{3} \mathrm{~km}$ ) and in Paper II (where we show that satellites of size $\sim 10^{3} \mathrm{~km}$ forming in the outer disk might be prevented from migrating into the inner disk). Thus, we compute "minimum mass" gas densities in the inner and outer disks based on the solid mass required to form Io, Europa (reconstituted), and Ganymede in the inner disk and Callisto in the outer disk. Inside of the centrifugal radius the average surface gas density $\Sigma$ then exceeds $10^{5} \mathrm{~g} \mathrm{~cm}^{-2}$ (corresponding to pressures $p \geqslant 0.1$ bar), which yields a vertical optical depth (not including dust) due to absorption by hydrogen molecules $\tau_{v} \sim \Sigma K_{\text {gas }}$ $\sim 1-10$, where $K_{\text {gas }} \sim 10^{-5}-10^{-4} \mathrm{~cm}^{2} \mathrm{~g}^{-1}$ is the Rosseland mean gas opacity (Mizuno, 1980; Lunine and Stevenson, 1982). Well outside the centrifugal radius, gas surface densities are in the range $10^{2}-10^{3} \mathrm{~g} \mathrm{~cm}^{-2}$, which results in a low vertical optical depth in the range $\tau_{v} \sim 0.001-0.01$.

While grains contribute to the opacity so long as their sizes are in the order of the infrared wavelength, coagulation may quickly lead to larger grain sizes (Weidenschilling and Cuzzi, 1993). One expects that the dust density is determined by a balance between the collisional dust production rate and the removal rate by drift, coagulation, accretion, and the like. In such an equilibrium state it may be appropriate to assume a power law size distribution. If we take this distribution to behave like $r_{p}^{-3}$ (as may result in a collisional situation where erosion replenishes small particles), and use the decoupling size $r_{p} \sim 10^{3} \mathrm{~cm}$ (smaller particles are strongly coupled to the subnebula gas) as the upper size cut-off (Weidenschilling, 1997; note that in that study the decoupling size was a somewhat smaller $\sim 1 \mathrm{~m}$ 
given the author's parameters for the solar nebula), we find that the mass in particles smaller than $\mu \mathrm{m}$ is $\sim 10^{-7}$ of the total mass in the disk. Using $\Sigma_{s}=(4 / 3) \rho_{s} r_{p} \tau_{\text {dust }}$, where $\tau_{\text {dust }}$ is the micrometer-sized dust optical depth, we find that for surface densities of solids $\Sigma_{s}<10^{3} \mathrm{~g} \mathrm{~cm}^{-2}$ we have $\tau_{\text {dust }}<$ 1 , where a grain density of $\rho_{s}=1.0 \mathrm{~g} \mathrm{~cm}^{-3}$ has been used. As a result, the dust optical depth may drop below unity once the satellites have accreted. However, the possibility remains that close to the planet hypervelocity collisions or the ablation of planetesimals passing through the disk lead to large dust production, the disk temperature is too high for water-ice condensation and efficient dust coagulation to take place (Supulver and Lin, 2000), or the relative velocity between particles is too high for efficient sticking (Suttner and Yorke, 2001). On the other hand, if the coagulation were inefficient it might be difficult to form satellites in the first place. Furthermore, sufficiently small particles may still coagulate even in the presence of turbulence (Suttner and Yorke, 2001).

In this study, we adopt the view that a weak turbulence regime is more likely to apply during satellite formation. In that case, coagulation may readily form even large particle sizes. As shown in Weidenschilling (1997), provided that the weak turbulence regime applies, coagulation in the solar nebula of particles up to the decoupling size takes place in a timescale of $\sim 10^{5}$ years. Given its shorter dynamical times, one might expect a shorter coagulation time for the subnebula; however, the exact nature of the scaling between the nebula and the subnebula remains to be studied. Also, the assumed starting condition for the solids as micrometersized grains may not apply to satellite formation. Nonetheless, we do not believe that this argument rules out the possibility that the inner disk (the outer disk has low gas density and long satellite accretion times) remains dusty throughout the formation of the satellites. Even so, it must be remembered that the temperature of the disk will approach the background temperature of the solar nebula at a distance of order tens of planetary radii. For this reason, one might expect that only regions close to the planet would be (weakly; Stone and Balbus, 1996) turbulent. We will return to this issue in Paper II. For now we consider the gaseous opacity alone and characterize the disk as thick or thin depending on whether the gaseous opacity is enough to make the disk optically thick or not.

If the opacity is small, individual grains are in radiative balance with the planet's luminosity and a temperature profile results with $T \propto r^{-1 / 2}$ (Pollack et al., 1977). On the other hand, when the opacity is high, viscous dissipation within the nebula plays a central role in heating the gas and driving its evolution (Lynden-Bell and Pringle, 1974). Though uncertain, the nonisothermal, optically thick portion of the subnebula may exhibit a temperature profile roughly of the form $T \propto r^{-1}$ as suggested by some solar nebula models (Cameron and Pine, 1973; Cameron, 1978b). Like previous authors (Lewis, 1972; Lunine and Stevenson, 1982), we characterize the temperature of the gas disk by choosing the current radial distance for Ganymede $\left(15 R_{\mathrm{J}}\right)$ as the location for the condensation of water ice $(250 \mathrm{~K})$.

There are several processes that might lead to satellite migration. Most important, the gas tidal torque on a fullsized Galilean satellite, when most of its water was already accreted, may alter the appropriate choice for the location of the condensation of water ice. Nevertheless, the closeness of Ganymede to Jupiter's centrifugal radius suggests that this satellite underwent limited radial migration (see Paper II for an explicit calculation). Furthermore, our model makes Ganymede from materials located between $\sim 15$ and $\sim 23 R_{\mathrm{J}}$ brought to its feeding zone by gas drag. Thus, setting the water-ice condensation temperature at $15 R_{\mathrm{J}}$ allows the material that went into Ganymede to have its complement of water in place. The resulting temperature profile is $T=3600 / x$, where $x=r / R_{\mathrm{J}}$, which is the same profile as that chosen by Lunine and Stevenson (1982). In the outer disk, the temperature is roughly given by the solar nebula temperature at the location of Jupiter $\sim 130 \mathrm{~K}$.

There are three mechanisms that can lead to the presence of an extended gas disk. The giant planet can spin-out a disk during its contraction phase (Korycansky et al., 1991). In a viscous disk with outward angular momentum transport, gas inside the radius of maximum viscous stress (probably located in the neighborhood of the centrifugal radius) drifts inward as it loses angular momentum, while gas outside this location expands outward as it receives angular momentum (Lynden-Bell and Pringle, 1974). An extended disk may also result from the tidal effects of the Sun on gas flowing from the Roche-lobe. Even though our scenario is generally consistent with the spin-out scenario of Korycansky et al. (1991), we do not rely on its validity. The spin-out model assumes opacities that are arguably too large if one takes into consideration coagulation and settling of dust grains (which provide the source of the opacity). For lower opacities, spin-out may not take place. On the other hand, gas viscosity may only lead to an extended outer disk if strong gas turbulence lasts a time comparable to the viscous time scale. Since we expect that far from the planet the gas flow became laminar soon after the end of planetary accretion, we do not favor the second mechanism. In this paper we favor the third possibility.

Determining the size of the outer disk requires careful calculation of the angular momentum of infalling gas. Because the material flows in from the Roche-lobe, the angular momentum of the resulting disk is sensitive to the solar tide. Numerical simulations suggest sizes as large as $\sim R_{\mathrm{H}} / 2$ (Bryden et al., 1999; Korycansky et al., 1991) or as small as $R_{\mathrm{H}} / 5$ (Korycansky and Papaloizou, 1996). It is also possible that the size of the outer disk is determined by the onset of an instability applicable to tidally distorted accretion disks (Goodman, 1993). Unfortunately, evaluation of this possibility is hampered by model uncertainties. Though a criterion for the onset of this instability has not been clarified, it appears to operate too far from the planet to be useful here. 
We adopt the choice $R_{\mathrm{H}} / 5 \sim 150 R_{\mathrm{J}}$ for the size of the outer disk (we choose the same fraction for Saturn, which corresponds to a larger distance $\sim 200 R_{\mathrm{S}}$ ). We justify this choice by noting that the inner irregular Jovian satellites are located at $160 R_{\mathrm{J}}$ just outside this distance. That is, we tie the size of the outer disk to the location of the inner irregular satellites. Too much gas beyond this point would have resulted in the inward drag of these objects. In our model, gas drag explains the absence of irregular satellites closer to Jupiter (in the case of Saturn a similar argument can be made for Phoebe at $215 R_{\mathrm{S}}$ ). Prior to collapse, Jupiter's envelope extended out to $\sim 300 R_{\mathrm{J}}$ and may have led to the capture of irregulars inside this radius (Pollack et al., 1979). The idea is for the envelope collapse to leave behind captured objects. After collapse, a disk was left behind that dragged in all the solids left in orbits $<150 R_{\mathrm{J}}$. Even if a different method is employed to capture at least some of the irregular satellites, we stress that their locations and eccentricities suggest a relatively sharp cut-off in the gas distribution of the outer disk. This cut-off is difficult to reconcile with a viscous medium. We attribute this observation to the low viscosity of the gas in the isothermal outer disk. In this regard, we note that Cuk and Burns (2002) use an extended disk to capture irregular satellites both into planetary orbits as well as into resonance. These authors choose a surface gas density profile consistent with that of our model (Cuk, private communication). The presence of an edge provides both the strong and weak gas regimes needed to explain capture into planetary orbits and resonance capture (Saha and Tremaine, 1993).

It is useful here to discuss planetary gap-opening in some detail. We can estimate the time scale for gap-opening by calculating the angular momentum $L_{\Delta}$ in an annulus of half-width $\Delta$. Given the planetary torque on this annulus $\dot{L}_{T}$, the time scale for gap-opening is given by $\tau_{\text {gap }} \approx L_{\Delta} / \dot{L}_{T}$. An analytical estimate for the gap-opening time scale can be obtained using the tidal torque formula (Lin and Papaloizou, 1993)

$$
\dot{L}_{T}=0.23\left(\frac{M_{P}}{M_{\odot}}\right)^{2} \Sigma a^{4} \Omega^{2}\left(\frac{a}{\Delta}\right)^{3},
$$

where the planetary feeding zone $\Delta$ must be larger than the scale-height of the nebula $H$, and the semi-major axis of the secondary is $a$. Assuming a constant gas surface density $\Sigma$, the angular momentum the planet must add or remove from the annulus of half-width $\pm \Delta$ in order to open up a gap is given by

$$
\begin{aligned}
L_{\Delta}= & 2 \pi \Sigma a^{3}\left(G M_{\odot} / a^{3}\right)^{1 / 2} \int_{0}^{ \pm \Delta}\left[\left(1 \pm \frac{\Delta}{a}\right)^{1 / 2}\right. \\
& \left.-\left(1+\frac{x}{a}\right)^{1 / 2}\right]\left(1+\frac{x}{a}\right) d x
\end{aligned}
$$

which can be written as

$$
L_{\Delta}=\pi \Sigma \Omega a^{4}\left[\frac{1}{5}\left(1 \pm \frac{\Delta}{a}\right)^{5 / 2}-\left(1 \pm \frac{\Delta}{a}\right)^{1 / 2}+\frac{4}{5}\right] .
$$

Then expanding to second order in $\Delta / a$, we find

$$
L_{\Delta} \approx \frac{1}{2} \pi \Sigma \Omega a^{2} \Delta^{2}
$$

The gap-opening time is then given by $\tau_{\text {gap }} \approx(\Delta / a)^{5} P / q^{2}$, where $q=M_{P} / M_{\odot}$ is the mass ratio of the secondary to the primary (Bryden et al., 1999). Unless $\Delta$ is several times the planet's Hill radius, accretion onto the planet will continue. For some nebula models (Lubow et al., 1999) gap-opening will fail to stop the accretion of a Jupiter mass $\left(1 M_{J}\right)$ giant planet. These workers estimate that it will take a $6 M_{J}$ planet to create a gap large enough with respect to its Roche-lobe to stop accretion onto the planet. Of course, it then becomes difficult to explain the mass of Jupiter. It should be pointed out that the above conclusion depends on the strength of the assumed turbulent viscosity of the nebula. Nevertheless, gas flowing through the gap is a significant issue that needs to be addressed. In our model, given its high specific angular momentum, this late arriving component simply adds gas and some condensables (what fraction of condensables is uncertain, though it is likely to be well below solar mixtures (Weidenschilling, 1997) to the outer disk after Jupiter has already accreted most of its mass. In any case, it is clear that the relevant size of the annulus $\Delta$ has to be large compared to the Roche-lobe of the planet in order to lower the mass rate accreted onto the planet. Using $\Delta \sim 0.2 a_{J}$ (which is about three times larger than the Hill radius for Jupiter), one obtains $\tau_{\text {gap }} \sim 380 P$, where $P$ is the period of Jupiter's orbit. This estimate is similar to the numerical value $\tau_{\text {gap }} \sim 320 P$ $\sim 4 \times 10^{3}$ years given by Bryden et al. (1999).

In the context of the satellites we are more interested in halting inward migration than we are in ending accretion. We expect accretion of the large, regular satellites to end when the disk has been depleted of condensables or when an outer satellite limits the inner satellite's supply of inward drifting satellitesimals. This differs from the solar nebula context where a minimum mass model may not apply (Lissauer and Stewart, 1993). It is possible, however, that gapopening also plays a role in regulating the growth of satellites. As we discuss in Paper II, we expect that at least the largest satellites truncated the gas disk in which they were formed, thus preventing gas outside their orbits from accreting to the planet. Bryden et al. (2000) and Kley (2000) discuss a process by which the gas between two giant planets may be cleared by the action of planetary torques, which may lead to subsequent evolution of the planets into resonant orbits as the gas disk evolves. Assuming acoustic waves dissipate in the neighborhood of the resonant locations where they are launched (see Paper II), we can obtain an analogous estimate of the time $\tau_{\text {dis }}$ it would take for gas 
Table 2

Surface density parameters

\begin{tabular}{lllclrrr}
\hline Planet & $\begin{array}{l}\sum_{\text {in }}{ }^{0} \\
\left(10^{4} \mathrm{~g} \mathrm{~cm}^{-2}\right)\end{array}$ & $\begin{array}{l}\sum_{\text {out }}{ }^{0} \\
\left(10^{4} \mathrm{~g} \mathrm{~cm}^{-2}\right)\end{array}$ & $r_{1}\left(R_{p}\right)$ & $r_{2}\left(R_{p}\right)$ & $R_{\text {in }}\left(R_{p}\right)$ & $R_{\text {out }}\left(R_{p}\right)$ & $\begin{array}{c}a_{1} \\
\left(10^{4} \mathrm{~g} \mathrm{~cm}^{-2}\right)\end{array}$ \\
\hline Jupiter & 51 & 0.31 & 20 & 26 & 14 & 87 & 36 \\
Saturn (3.7) & 10 & $6.5 \times 10^{-2}$ & 25 & 37 & 16 & 115 & 5.4 \\
Saturn (Iap) & 11 & $7.7 \times 10^{-3}$ & 25 & 41 & 18 & 117 & 5.8 \\
Uranus & 1 & - & - & - & - & - & -13 \\
\hline
\end{tabular}

${ }^{\mathrm{a}}$ Unsmoothed values. The parameter $\mathrm{b}_{1}=\ln \left[\left(\sum_{\mathrm{in}}^{0} R_{\mathrm{in}} r_{2}\right) /\left(\sum_{\mathrm{out}}^{0} R_{\mathrm{out}} r_{1}\right)\right] / \ln \left(r_{2} / r_{1}\right)$.

dissipation between Io and Ganymede by finding the location $a_{\mathrm{dis}}$ inside of Ganymede and outside of Io such that both Io and Ganymede will open a gap extending to this same position in the same amount of time. Using Eqs. (2) and (4) we obtain $a_{\mathrm{dis}} \approx 9 R_{\mathrm{J}}$ and $\tau_{\mathrm{dis}} \approx 1 \times 10^{5}$ years (for the sake of comparison, we note that an equivalent calculation for Jupiter and Saturn yields a times cale of $\sim 5 \times 10^{5}$ years). This time scale may be longer than the (inner disk) satellite formation time, but it is considerably shorter than the planetary cooling time $\sim 10^{6}$ years (Pollack et al., 1976). Hence, the gas disk between Io and Ganymede may dissipate before the water content in the disk has a chance to condense. It is also tempting to ascribe the resonances of Io, Europa, and Ganymede to the aforementioned process of gap opening followed by disk evolution; however, for the Galilean satellites it is more likely that the resonant configuration is due to gas drag capture or tidal evolution (significant disk evolution might result in the loss of the satellites due to Type II migration, though there may be ways around this).

So far we have described a circumplanetary disk (or subnebula) with an optically thick (even when grains are excluded) inner region inside the centrifugal radius and an optically thin outer region outside the centrifugal radius and extending to a significant fraction of the Roche-lobe. To characterize the transition region between the inner and outer disks let us first assume that planetary accretion drives subnebula turbulence. If we choose a time scale for gas evolution in the presence of accretion-driven turbulent viscosity to be the gap-opening time $t_{0} \sim 1000$ years, and we use a length scale on the order of the centrifugal radius $R_{0}$ $\sim r_{c} \sim 15 R_{\mathrm{J}}$, we get $\nu=R_{0}^{2} t_{0} \sim 10^{11}-10^{12} \mathrm{~cm}^{2} \mathrm{~s}^{-1}$. This gives $\alpha=\nu \Omega / c^{2} \sim 10^{-4}-10^{-3}$ at that location. This leads us to expect that following planetary accretion of the location of the gas density drop-off will be outside but perhaps close to the location of the centrifugal radius. Unfortunately, the size of the transition region is unknown. However, it must be larger than the subnebula scale-height to avoid becoming Rayleigh unstable (Lin and Papaloizou, 1993). This sets our choice for the maximum surface density gradient in the transition region.

Following the completion of accretion, the gas turbulence will subside and the subnebula disk will cool in a Kelvin-Helmholtz time scale $\sim 10^{4}$ years (Stevenson et al., 1986). At the outer edge of the inner disk, where the subnebula temperature approaches the background temperature of the solar nebula, the flow will become laminar, with very low viscosity and long evolution times. Closer in (inside the orbit of Callisto), remnant turbulence may be driven by the entropy gradient due to the planetary gravitational energy release (Klahr and Bodenheimer, 2001). If we estimate the postaccretion turbulence inside Ganymede to be $\alpha \sim 10^{-6}$ $10^{-4}$ then the time scale for Jupiter's inner disk to become optically thin is $\sim 10^{4}-10^{5}$ years (see Fig. 4). Thus, the inner disk may continue evolving until a gas density of $\sim 10^{4} \mathrm{~g}$ $\mathrm{cm}^{-2}$ (which corresponds to a pressure of $\sim 3 \times 10^{-3}$ bar at $15 R_{J}$ ) is reached. At that time the inner disk may become optically thin and the turbulence may die down. Hence, it may be difficult to lower the density of the disk below gaseous optical depth of order unity by gas turbulence alone (unless small dust particles are somehow replenished and the dust optical depth remains high on viscous time scales, which is in doubt given the shorter coagulation time scales). For the outer disk, we expect weak turbulence close to the midplane early on, driven by the presence of a dust and rubble layer (due to the particle shear layer (Cuzzi et al., 1993), and laminar flow at later times.

As a starting condition we choose a simple model where the gas density follows a simple $1 / r$ dependence inside of $20 R_{\mathrm{J}}$ and outside of $26 R_{\mathrm{J}}$. The transition region has a width of about $\sim 2 H_{c}$, where $H_{c}$ is the subnebula scale-height at the centrifugal radius of the primary. This choice ensures that the gradient in gas density is not so steep as to lead to a Rayleigh-Taylor instability (e.g., Lin and Papaloizou, 1993). Our density profile is given by

$$
\Sigma(r)= \begin{cases}\Sigma_{\text {in }}^{0}\left(R_{\text {in }} / r\right), & r<r_{1} ; \\ a_{1} r^{-b 1}, & r_{1}<r<r_{2} ; \\ \Sigma_{\text {out }}^{0}\left(R_{\text {out }} / r\right), & r>r_{2},\end{cases}
$$

where the relevant parameters for our various model choices are presented in Table 2.

In Fig. 2, we plot the surface density as a function of radial location for our model for Jupiter and Saturn as well as other models in the literature. Though our model bears a close relation to the standard minimum mass model, it differs in the distribution of mass in that we allow for an extended low density component. For Jupiter, the mass of Callisto is distributed in the outer disk. For Saturn, we do not spread the mass of Titan out to Iapetus as is done in the 


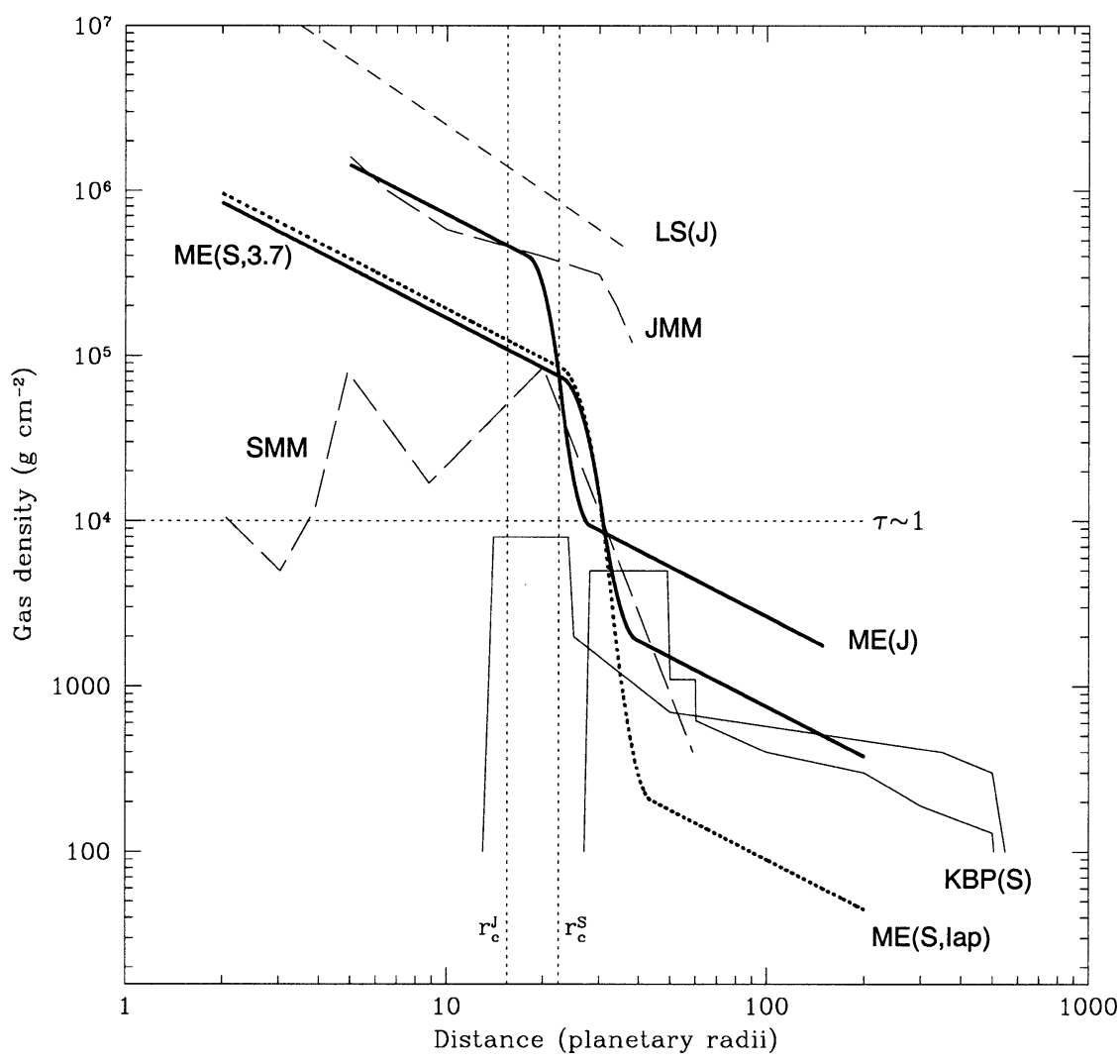

Fig. 2. Comparison of gas surface density for our models as well as previous models for the saturnian and jovian systems. LS(J): Lunine and Stevenson (1982). JMM/SMM: The minimum mass model for Jupiter and Saturn respectively of Pollack et al. (1994). KBP: Spin-out models of Korycansky et al. (1991) for Saturn. ME(J): Our minimum mass density profile for Jupiter after reconstitution of water ice for Io and Europa. Disk size extends to $\sim 150 R_{\mathrm{J}}$. ME(S): Solid line indicates our model for Saturn in which the mass of solids in the subnebula is taken to be 3.7 times less than that of Jupiter. Dotted line indicates a model in which just enough mass is placed outside of the orbit of Iapetus to form it. The locations of both Jupiter's and Saturn's centrifugal radii are labeled. The dotted horizontal line corresponds to a gas surface density such that the gas optical depth is $\tau \lesssim 1$.

SMM model; rather, we expect that most of the mass of Titan came from the inner disk which extends roughly out to the position of Hyperion. It should be noted that in our model the size of the inner disk of Jupiter and Saturn scale with the size of the planet's Hill radius, which leads to a more extended disk for Saturn. The surface density of Saturn's disk is smaller both because there is less mass in the satellite system and also because the mass is more spread out. We have plotted two curves for our Saturn model which differ mostly in the treatment of the outer disk. The solid curve corresponds to a model where we keep a constant mass ratio of 3.7 for the masses of the outer and inner disks of Jupiter with respect to the outer and inner disks of Saturn. The dotted curve was determined by the amount of material needed to make Iapetus out of the condensables present in the outer disk assuming a cosmic mixture (see Sect. 6). The curves labeled KBP correspond to cases for Saturn and should be compared to our Saturn curves. Notice the presence of an extended component out to $R_{\mathrm{H}} / 2 \sim 500 R_{\mathrm{S}}$ for the KBP model. These correspond to the spin-out scenario of Korycansky et al. (1991). The curve labeled LS(J) corresponds to the work of Lunine and Stevenson (1982), which extended an adiabat from the planet to form a subnebula with several times more mass in solids than is present in Jupiter's satellite system. In Fig. 3, we plot the temperature profile of the disks of Jupiter and Saturn as a function of radial location. Saturn's temperature profile will be described more fully in Section 6.

For the optically thick portion of the disk, where the temperature profile is determined by viscous dissipation, we have

$$
\Sigma \nu r^{2}\left(\frac{d \Omega}{d r}\right)^{2}=2 \sigma_{S B}\left(T^{4}-T_{0}^{4}\right)
$$

where $\sigma_{S B}$ is the Stefan-Boltzmann constant, $T$ is the photospheric temperature, and $T_{0}$ is the nebula background temperature. In Fig. 4 we use the density profile of Fig. 2, the temperature profile of Fig. 3, the above equation, and the equation $t \sim r^{2} / \nu$ to plot the viscosity, the diffusion time, and the turbulence coefficient $\alpha=\nu \Omega / c^{2}$, where $c$ is the speed of sound, as a function of position in the disk for Jupiter and Saturn (the Iapetus' minimum mass model was used to set the surface density of Saturn's outer disk, see Sect. 6). A viscosity bump is seen in the Jupiter curve but not the Saturn curve due to the difference in the temperature 


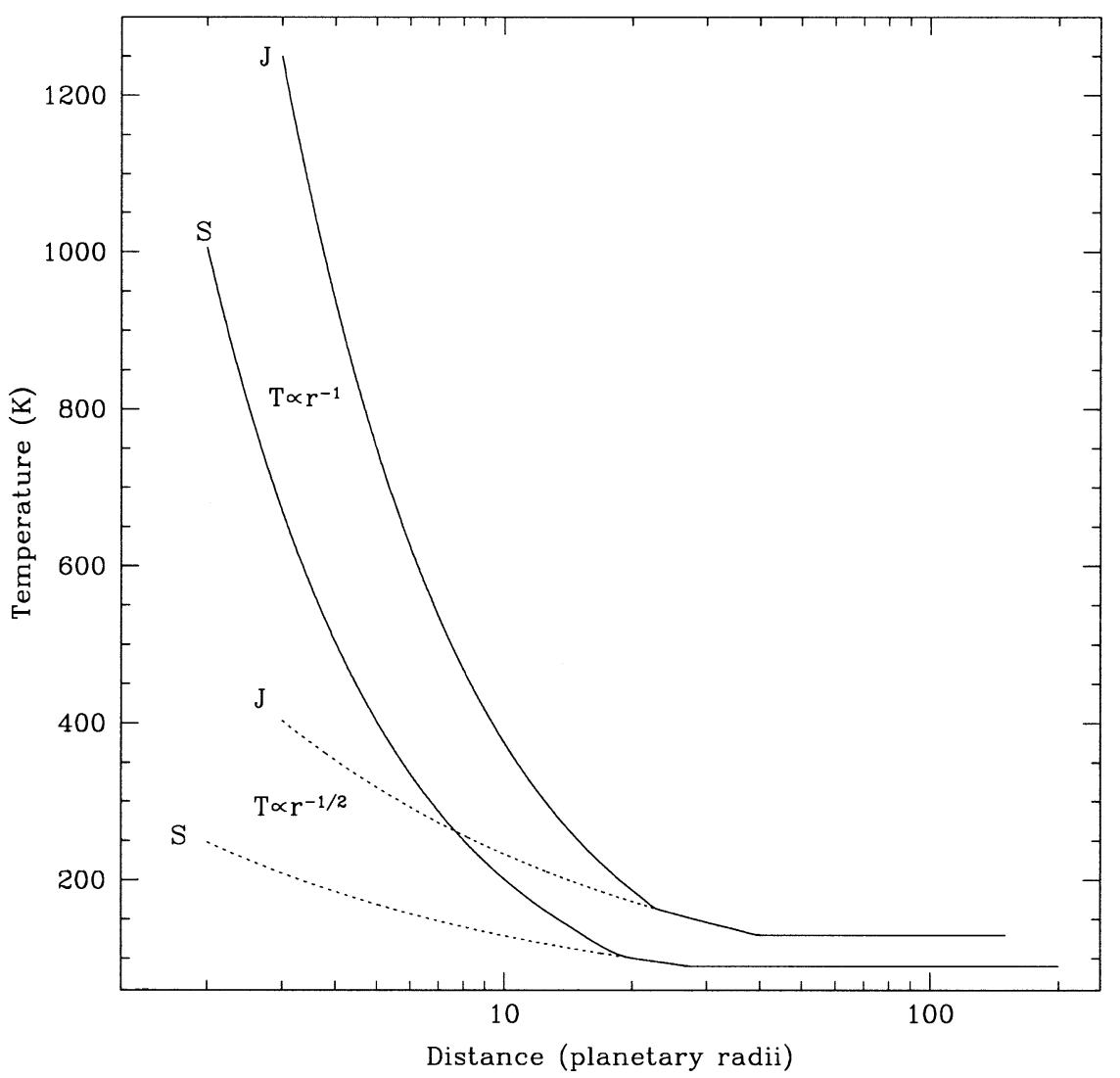

Fig. 3. Temperature profiles used in our model. Inner disk temperature varies like $r^{-1}$. Transition in behavior to $r^{-1 / 2}$ occurs around the centrifugal radius of both planets. Temperatures at greater distances in the outer disk are constant. The temperatures in the outer disk are taken to be that of the equilibrium solar nebula temperature $\left(T_{e} \approx 280 \sqrt{1 A U / r}\right)$, which is roughly $130 \mathrm{~K}$ for Jupiter and $90 \mathrm{~K}$ for Saturn. A temperature of $250 \mathrm{~K}$ is set to coincide with the position of Ganymede for Jupiter, and Rhea for Saturn.

profiles in the transition region, but its significance, if any, is unknown.

From Fig. 4 one obtains an inner disk $\alpha \sim 10^{-6}-10^{-5}$. However, it is possible that our model significantly overestimates the inner disk gas surface density. Furthermore, radial migration both of satellitesimals and embryos introduces uncertainty in the disk temperature at the present position of Ganymede and Titan. Finally, it is unclear whether the turbulence generated by the formation of large scale vortices as advocated by Klahr and Bodenheimer (2001) can be described by an $\alpha$ model prescription. Given these uncertainties, it is possible to consider larger values $\alpha$ $\sim 10^{-4}$. At any rate, Fig. 4 argues in favor of a weak $\alpha$ regime.

It is important to point out that our nebula may not be vertically isothermal. The temperatures at the midplane may be significantly larger than the photospheric temperatures (though the degree to which this is the case depends on the details of the turbulent dissipation model). Hence, it is unlikely that satellite accretion can be thought of as a homogeneous process, with the rock and ice components accreting at the same time. Nevertheless, the ice/rock ratio of the large satellites indicates that this complication does not prevent accretion of either component. To the extent that inhomogeneous accretion can affect the final structure of the satellites, it will do so in the inner disk, where the cooling times are significantly longer and the accretion times shorter. Though the satellite itself is in a hotter region of the disk, this may not pose a problem. A satellite may accrete water trapped in satellitesimals that drift in from cooler regions of the disk or accrete the water component following the accretion of the rock component, once the disk has cooled.

\section{Galilean satellite accretion and evolution}

In analogy to gas-free planetary accretion, we begin the problem of satellite accretion by calculating characteristic sizes of satellitesimals and satellite embryos for our disk parameters assuming a satellitesimal density of $\rho_{s}=1.5 \mathrm{~g}$ $\mathrm{cm}^{-3}$. Though our problem differs markedly from one in which the satellites are accreted in the absence of gas, we will show later that the characteristic sizes one obtains in the presence of gas are roughly consistent with the ones we give below, which are meant only as an indication of typical object sizes. The characteristic length scale and mass scales over which the disk's self-gravity dominates shear are ap- 

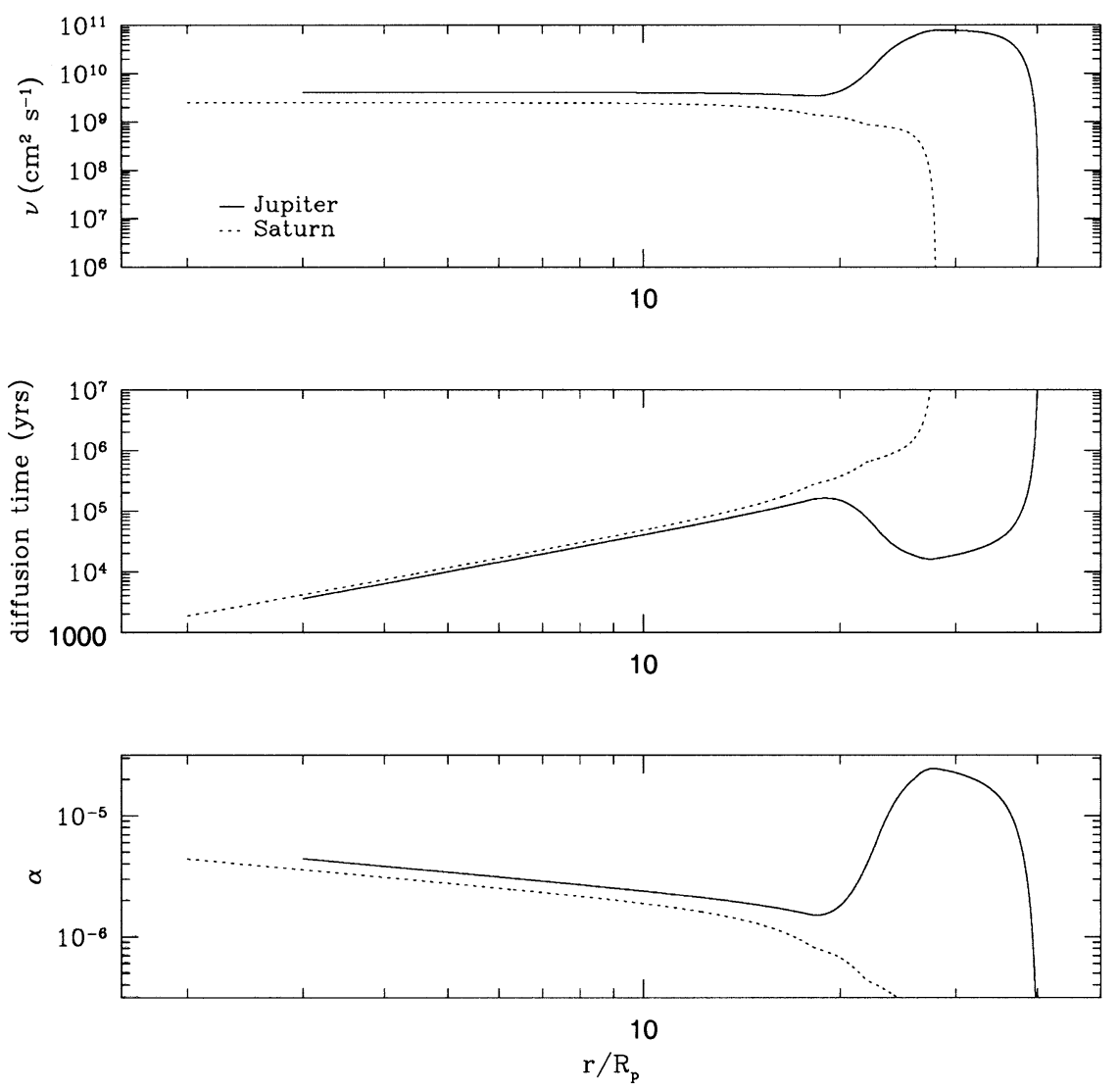

Fig. 4. A plot of viscosity, diffusion time, and turbulence coefficient for Jupiter's and Saturn's inner disk using our temperature profiles (see Fig. 2). Low viscosities correspond to weak turbulence and long evolution times. The bump present in the Jupiter curve but not the Saturn curves are due to the difference in the temperature profiles in the transition region.

proximately $l_{1}=r_{H}$, where $l_{1}=\left(m_{1} / \pi \Sigma_{s}\right)^{1 / 2}$ and $r_{H}=$ $a\left(m_{1} / 3 M_{\mathrm{J}}\right)^{1 / 3}$ is the Hill radius of a satellitesimal of mass $m_{1}$ $=\pi \Sigma_{s} a^{2}\left(\mu_{d} / 3\right)^{2}$, where $\mu_{d}=\pi \Sigma_{s} a^{2} / M_{J}$ (Goldreich and Ward, 1973). Using a surface density of solids of $\Sigma_{s} \sim 5 \times$ $10^{3} \mathrm{~g} \mathrm{~cm}^{-2}$ for the inner disk of radius $\sim 15 R_{\mathrm{J}}$, we obtain $m_{1}$ $\sim 1.6 \times 10^{17} \mathrm{~g}$, which corresponds to a satellitesimal radius of $\sim 3 \mathrm{~km}$ and $l_{1} \sim 32 \mathrm{~km}$. The second characteristic mass and radial scale, $l_{2}=m_{2} /\left(4 \pi a \Sigma_{s}\right)$, is the distance over which Keplerian shear can force close encounters among satellitesimals $l_{2}=r_{H}$. This gives $m_{2}=8 \pi \Sigma_{s} a^{2}\left(\mu_{d} / 3\right)^{1 / 2}$ (Hourigan and Ward, 1984). Using the same surface density as before, we get $m_{2} \sim 7.6 \times 10^{24} \mathrm{~g}$, which corresponds to an embryo radius of $\sim 1100 \mathrm{~km}$ and $l_{2} \sim 1.2 \times 10^{4} \mathrm{~km}$.

Assuming satellite formation is controlled by binary accretion of satellitesimals, we can write the time scale for accretion as (Safronov, 1969; Lissauer and Stewart, 1993; Ward, 1993)

$$
\tau_{\mathrm{acc}} \sim \frac{\rho_{s} r_{s}}{\sum_{s} \Omega} F_{g}^{-1}
$$

where $F_{g}$ is the gravitational focusing factor. In the case of planetary accretion this focusing factor can be quite large during runaway growth. For satellites, however, the Hill radius $r_{I I}$ is never much larger than the physical radius $r_{s}$.
An upper limit to the enhancement factor can be obtained (e.g., Weidenschilling, 1974): $F_{g} \sim\left(r_{H} / r_{s}\right)^{3 / 2} \sim O(10)$ for Ganymede. Then we can use Eq. (8) to obtain

$$
\begin{aligned}
\tau_{\mathrm{acc}} \sim & 4.8\left(\frac{\rho_{s}}{1 \mathrm{~g} \mathrm{~cm}^{-3}}\right)\left(\frac{r_{s}}{100 \mathrm{~km}}\right)\left(\frac{10^{4} \mathrm{~g} \mathrm{~cm}^{-2}}{\Sigma_{s}}\right) \\
& \times\left(\frac{a}{20 R_{\mathrm{J}}}\right)^{3 / 2} F_{g}^{-1} \text { years. }
\end{aligned}
$$

Given a surface density of solids of $\Sigma_{s} \sim 10^{3} \mathrm{~g} \mathrm{~cm}^{-2}$, this formula predicts a time scale of formation of $\sim 100$ years for a Galilean-sized satellite. This, however, assumes that all the solids in the disk are in the form of satellitesimals. Let us assume for the sake of discussion that at some time early on all the solids in the inner disk are in satellitesimals of characteristic size $\sim 1 \mathrm{~km}$. In that case, the time for drag to completely clear the inner disk of solids (see Eq. (12) would be a few years! Therefore, unless most of the mass resides in large satellitesimals, it is unlikely that the binary accretion time scale controls the process of satellite formation. Instead, we form satellites first by the sweep-up of dust and rubble, followed by the accretion of inwardly migrating satellitesimals once a significant fraction of the solids in the disk have aggregated to $100-\mathrm{m}$ or larger objects. 
a

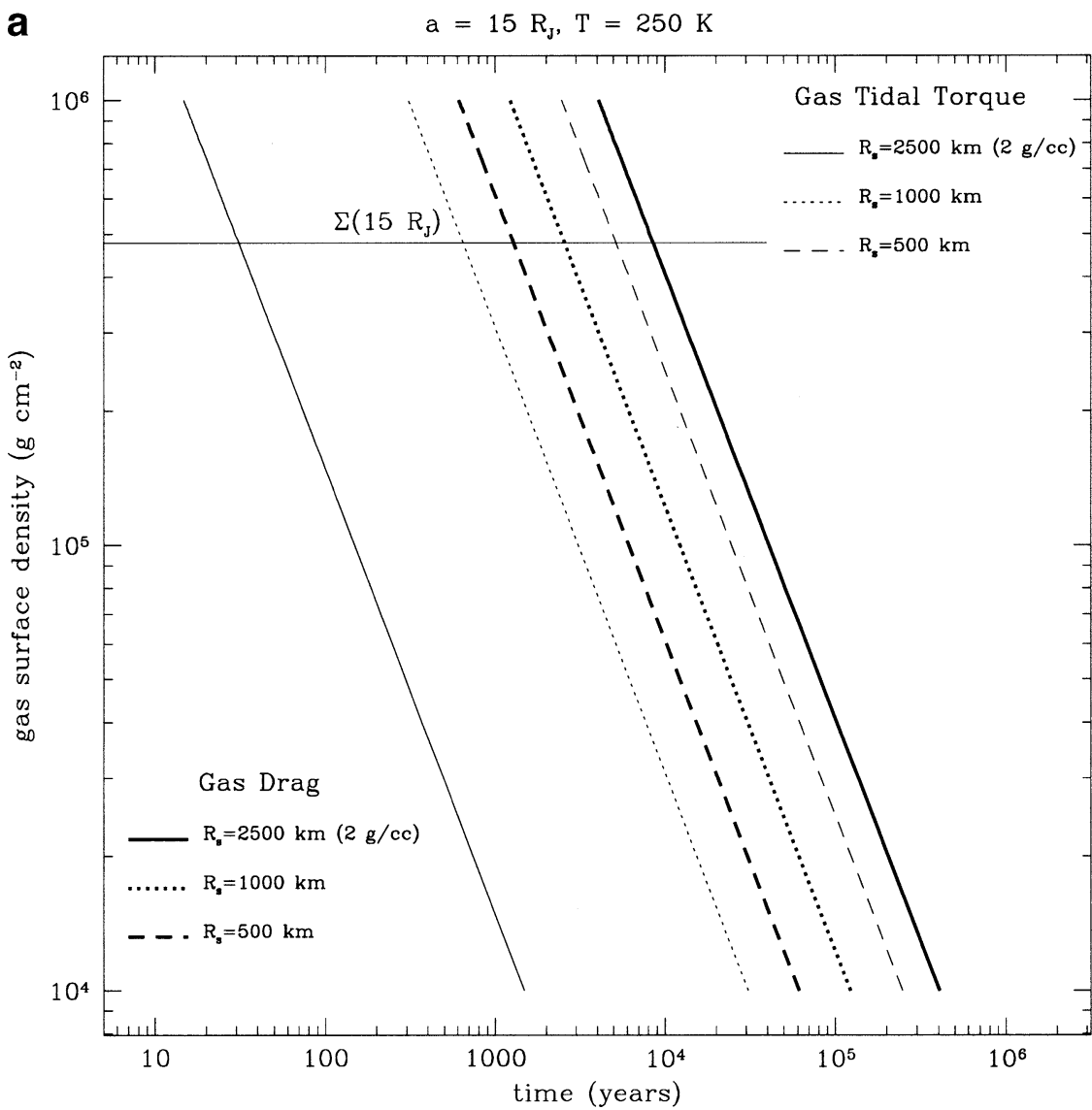

Fig. 5. (a) Orbital decay times of satellitesimals of various sizes due to gas drag and gas tidal torque at $15 R_{\mathrm{J}}$. Unless otherwise indicated the density is $\rho_{\mathrm{s}}=1.5 \mathrm{~g}$ $\mathrm{cm}^{-3}$. The gas surface density for our models is indicated in the plot. The decay times of smaller objects are dominated by gas drag, while larger objects are controlled by gas tidal torque, with the transition occuring between 500 and $1000 \mathrm{~km}$. (b) Orbital decay times of satellitesimals of various sizes due to gas drag and gas tidal torque at $50 R_{\mathrm{J}}$. Unless otherwise indicated the density is $\rho_{\mathrm{s}}=1.5 \mathrm{~g} \mathrm{~cm}^{-3}$. The gas surface density for our models is indicated in the plot.

In Fig. 5, we calculate the orbital decay time scales due to gas drag and tidal torque of a proto-satellite at $15 R_{\mathrm{J}}$ (a) and $50 R_{\mathrm{J}}$ (b) for a range of gas densities $\rho_{g}$. Because the gas is partly supported by gas pressure, its orbital velocity $v_{\text {gas }}$ is slightly lowered with respect to the Keplerian circular velocity $v_{K}$. An object orbiting at Keplerian speed will therefore experience head-wind, suffer drag, and drift toward the primary. A measure of the difference between the Keplerian velocity and the drag velocity is given by

$$
\eta=\frac{v_{K}-v_{g a s}}{v_{K}} \approx-\frac{r}{2 \rho_{g} v_{K}^{2}} \frac{\partial p}{\partial r} \sim\left(\frac{c}{v_{K}}\right)^{2},
$$

where $p$ is the gas pressure, $r$ is the radius, and $c$ is the speed of sound. The time scale for orbital decay due to gas drag is given by

$$
\tau_{\mathrm{gas}}=\frac{4 \rho_{s} r_{p} v_{K}}{3 C_{D}(\Delta v)^{2}} \frac{2 c}{\Omega \Sigma},
$$

where $\rho_{s}$ and $r_{p}$ are the satellitesimal density and radius, $C_{D}$ $=0.44$ is the Stokes flow regime drag coefficient for high Reynolds number, $\Omega$ is the orbital frequency, $\Sigma$ is the gas surface density, and $\Delta v=\eta v_{K}$. The stopping time $t_{s}$ can be written in terms of the time scale for radial migration $t_{s}=$ $2 \Delta v \tau_{\text {gas }} / v_{K}$ (Weidenschilling, 1988). We can write Eq. (11) in the form

$$
\begin{aligned}
\tau_{\mathrm{gas}} \approx & 22\left(\frac{\rho_{s}}{1 \mathrm{~g} \mathrm{~cm}^{-3}}\right)\left(\frac{r_{p}}{1 \mathrm{~km}}\right)\left(\frac{130 K}{T}\right)^{3 / 2} \\
& \times\left(\frac{10^{5} \mathrm{~g} \mathrm{~cm}^{-2}}{\Sigma}\right) \text { years. }
\end{aligned}
$$

The torque time scales are calculated using the formulation of Ward (1997). However, ignoring some technicalities for now, the tidal torque exerted on the disk in the vicinity of an $m$ th order Lindblad resonance (Goldreich and Tremaine, 1978,1979 ) is given by

$$
\mathcal{T}_{m}=-\pi m \Sigma \Psi_{m}^{2}\left(r \frac{d D}{d r}\right)^{-1}
$$

where $\Psi_{m}$ is the forcing function of the satellite, and $D$ is a function of the difference in local epicyclic frequency of the disk and the Doppler-shifted forcing frequency. An estimate 


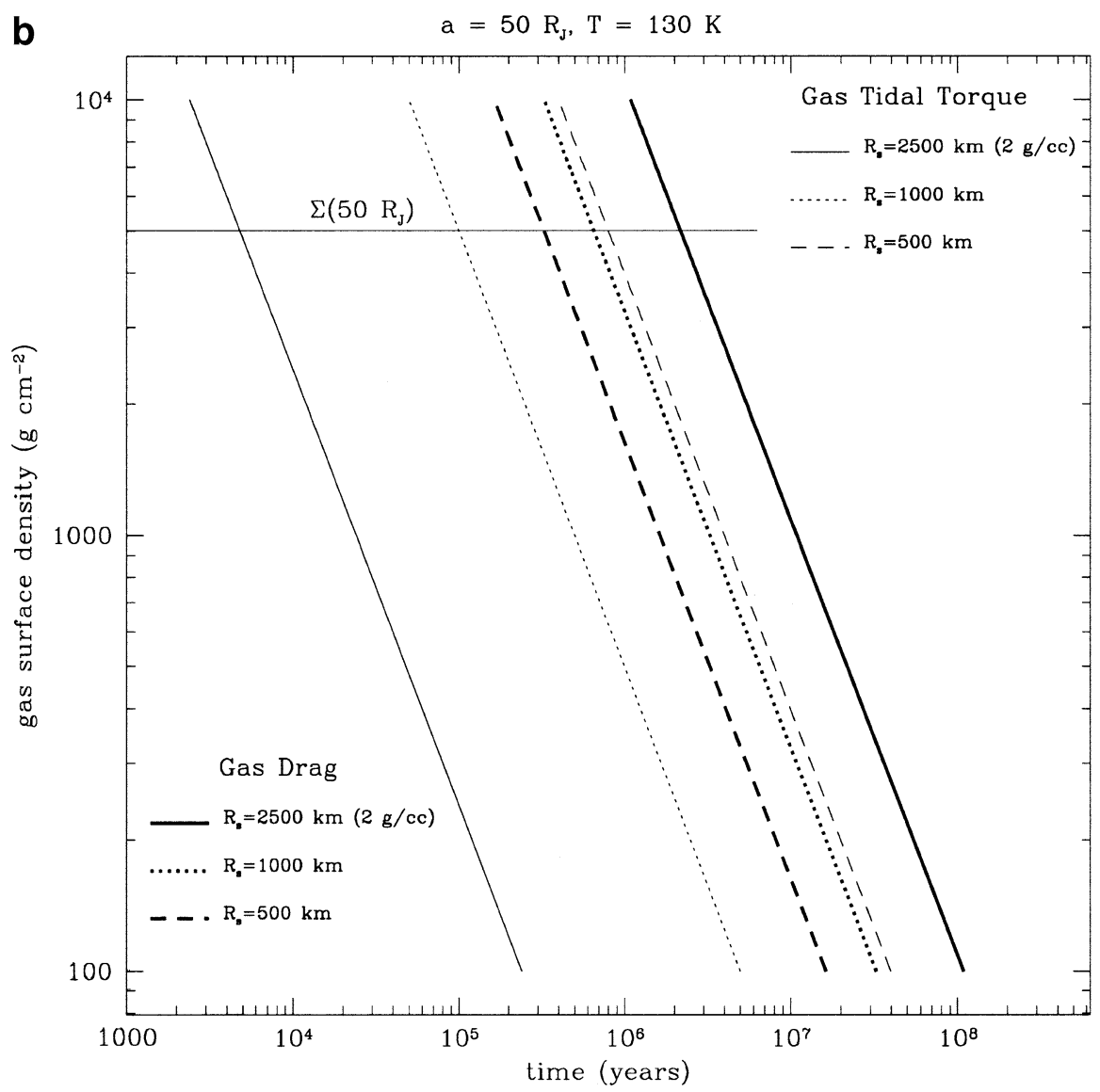

Fig. 5 (continued)

of the forcing function that works well in cases far from the transition region is $\Psi_{m} \sim(2 m-1) G M_{s} / a$, where $M_{s}$ is the satellite mass and a is its position, with $m \sim a / H$, the resonance where most of the tidal torque is deposited (Takeuchi et al., 1996), and $r d D / d r \sim 3(m-1) \Omega^{2}$. We can write the orbital decay time scale due to the tidal torque $m \times$ $\mathcal{T}_{m}$ in terms of our subnebula parameters

$$
\begin{aligned}
\tau_{\text {torq }} \approx & 3.2 \times 10^{4}\left(\frac{1 \mathrm{~g} \mathrm{~cm}^{-3}}{\rho_{s}}\right)\left(\frac{100 \mathrm{~km}}{r_{p}}\right)^{3} \\
& \times\left(\frac{T}{130 \mathrm{~K}}\right)^{3 / 2}\left(\frac{10^{5} \mathrm{~g} \mathrm{~cm}^{-2}}{\Sigma}\right)\left(\frac{a}{R_{\mathrm{J}}}\right) \text { years. }
\end{aligned}
$$

Using Eqs. (12) and (14), we can get a rough estimate of the transition size $r_{T}$ of a satellitesimal where gas drag and gas tidal torque in a smoothly varying disk are similar,

$$
r_{T} \sim 200\left(\frac{1 \mathrm{~g} \mathrm{~cm}^{-3}}{\rho_{s}}\right)^{1 / 2}\left(\frac{T}{130 \mathrm{~K}}\right)^{3 / 4}\left(\frac{a}{R_{J}}\right)^{1 / 4} \mathrm{~km},
$$

which yields a size of $\sim 550 \mathrm{~km}$ for a satellitesimal with density $\rho_{s}=1.5 \mathrm{~g} \mathrm{~cm}^{-3}$ at $15 R_{\mathrm{J}}$ (an exact calculation gives a value closer to $\sim 650 \mathrm{~km}$ ). For larger sizes, the gas tidal torque dominates the satellite's evolution. Since the estimate given by Eq. (14) does not take into account that the net tidal torque must be weighted by a measure of the tidal torque asymmetry between inner and outer torques, this expression overestimates the strength of the net tidal torque. Actual torque values used to produce the figures are calculated by summing over inner and outer resonances out to a value of $m \gg a \Omega / c{ }^{1}$ Such a tidal torque produces mostly inwardly migrating satellites (Ward, 1997; see Paper II for a discussion of the tidal torque).

Fig. 5 considers the evolution of satellites with $r_{s}=500$, 1000 , and $2500 \mathrm{~km}$ as a result of gas drag and tidal torque separately. In Fig. 5a, we show the orbital decay time scale for an object located at $15 R_{\mathrm{J}}$ for a range of gas densities appropriate to the inner disk $\left(\Sigma=10^{4}-10^{6} \mathrm{~g} \mathrm{~cm}^{-2}\right)$. The decay times due to gas drag are typically shorter than those due to torque; however, for the larger objects, the tidal torque begins to dominate their evolution. In the inner disk the transition takes place for objects of size $\sim 1000 \mathrm{~km}$. Similarly in Fig. 5b, we show the orbital decay of an object

\footnotetext{
${ }^{1}$ But note that the gas tidal torque migration rates may be overestimated; see Paper II.
} 
starting at $50 R_{\mathrm{J}}$ where the gas density is much lower $(\Sigma=$ $10-1000 \mathrm{~g} \mathrm{~cm}^{-2}$ ). From this figure, it is clear that survival of a satellite in a gaseous medium (where most of the mass and angular momentum resides in the gas component) is an issue for objects of all sizes.

We begin by assuming that satellitesimals are formed by particle aggregation (Weidenschilling and Cuzzi, 1993). Though a gravitational instability (Goldreich and Ward, 1973) probably did not take place (Weidenschilling, 1988; Cuzzi et al., 1993), it is still possible that other instabilities did (Goodman and Pindor, 2000). Once satellitesimals form they quickly settle down to the subnebula midplane and continue to grow by dust and rubble sweep-up or by accretion of other satellitesimals. Assuming that most of the mass is in particle sizes small enough to be entrained in the gas, we can write the growth time scale due to dust and rubble sweep-up as

$$
\tau_{\text {sweep }}=\frac{4 \rho_{s} r_{p}}{3 \bar{\rho}_{p} \Delta v_{p}},
$$

where $\bar{\rho}_{p}$ is the average particle density. We can also write this equation in the form

$$
\begin{aligned}
\tau_{\text {sweep }} \approx & 0.58\left(\frac{\rho_{s}}{1 \mathrm{~g} \mathrm{~cm}^{-3}}\right)\left(\frac{r_{p}}{1 \mathrm{~km}}\right)\left(\frac{a}{R_{\mathrm{J}}}\right) \\
& \times\left(\frac{130 \mathrm{~K}}{T}\right)^{1 / 2}\left(\frac{10^{5} \mathrm{~g} \mathrm{~cm}^{-2}}{\Sigma}\right)\left(\frac{H_{p}}{H}\right) \text { years, }
\end{aligned}
$$

where $H_{p}$ is the particle scale-height, $H$ is the subnebula scale-height, and we have assumed a two-population particle size distribution where large particles move at essentially Keplerian speeds while the small particles are entrained in the gas. We have also assumed that the dust density is less than the gas density, and so the head-wind is not lowered by the dust concentration. That is, we let $\Delta v_{p} \approx$ $\Delta v \approx \eta v_{K}$. Allowing for $H_{p}<H$, the sweep-up time scale is smaller than the drag time scale for $a<38 R_{\mathrm{J}}$; that far from the planet our model is optically thin, isothermal, and quiescent. As a result, in the outer disk we expect $H_{p} \ll H$ (see Sect. 5). In the inner disk we expect $H_{p}<H$ during most or all of the satellite accretion process. Quite generally, then, our weak turbulence model yields shorter sweep-up times than drag times, thus favoring the formation of satellites. In the inner disk (inside of $15 R_{\mathrm{J}}, T=250 \mathrm{~K}$ ), where the temperature is inversely proportional to the radial location, the ratio of the sweep-up time to the gas drag time is independent of semi-major axis and particle size $\tau_{\text {sweep }}$ / $\tau_{\text {gas }} \sim H_{p} / H<1$. This result makes it possible to form satellites of any size $<1000 \mathrm{~km}$ (such that gas drag dominates their inward migration) at any radial location in the inner disk.

In the presence of turbulence, balance is established between the rate of diffusion of dust due to turbulence and the rate of settling due to gravity. The scale height of dust is then given by

$$
H_{p} \approx\left(\frac{\nu}{S c} \frac{C_{H}}{\Omega^{2} t_{s}}\right)^{1 / 2}
$$

where the Schmidt number is approximately given by $S c=$ $1+\Omega t_{s}$, and $C_{H} \approx 1$ is a constant (Dubrulle, 1993; Cuzzi et al., 1993). We expect accretion to take place under weak turbulence with $\alpha \sim 10^{-6}-10^{-5}$, which corresponds to turbulent viscosity $\nu \sim 10^{10} \mathrm{~cm}^{2} \mathrm{~s}^{-1}$ (see Fig. 4). For $1-\mathrm{cm}$ particles we get $H_{p} \sim 0.2 \mathrm{H}$. Then the sweep-up time scale for a 1000-km embryo becomes $\tau_{\text {sweep }} \sim 10^{3}\left(H_{p} / H\right) \sim 200$ years. Notice also that dust of size $1 \mathrm{~cm}$ will diffuse a distance $d \sim(t v / S c)^{1 / 2} \sim 0.1 R_{\mathrm{J}}$ in a time $t=2 \pi a / \Delta v$ due to gas turbulence. Since this is much larger than $\sim 1000 \mathrm{~km}$, the embryo will not clear its lane.

This time scale is likely to overestimate the time it would take to form such a satellite embryo for three reasons. First, dust will coagulate and settle to the midplane, thus lowering the sweep-up time. Second, the gas turbulence itself may die down for optically thin disks. Third, an embryo will also grow due to capture of inwardly migrating satellitesimals. Thus, satellite embryos can be made sufficiently quickly to survive inward migration due to gas drag.

As we noted before, for objects larger than $1000 \mathrm{~km}$, migration due to gas tidal torque becomes dominant over migration due to gas drag. Since the orbital decay time due to torque of an embryo-sized object is again about $10^{3}-10^{4}$ years, one might be tempted to simply continue sweeping up dust and rubble to form a full-sized satellite. However, so far we have assumed a large reservoir of dust and rubble such that the surface density to be swept did not change as the embryo grew. Clearly, as the surface density of dust and rubble decreases so will the efficiency of this process. In any event, dust coagulation and sweep-up and decay of gas turbulence make it likely that embryos grew to satellite size by accretion of satellitesimals.

We now define the feeding zone of a satellite embryo. During closest approach, a satellite embryo will pump the eccentricity of a previously circular satellitesimal by the amount $e=2.24\left(m_{s} / M_{J}\right)\left(a / s_{l}\right)^{2}$ (Julian and Toomre, 1966), where $m_{s}$ is the mass of the embryo and $s_{l}$ is the separation between the two objects. Crossing orbits will subsequently result if $s_{l}=a e$. Using this condition we obtain $s_{l}=$ $a\left(2.24 m_{s} / M_{J}\right)^{1 / 3}$ for the size of the embryo's feeding zone (i.e., we take the feeding zone to be $\sim 2 r_{H}$; this is slightly more conservative than the value for the feeding zone $2 \sqrt{3} r_{H}$ one obtains using Jacobi's constant and asking that the separation between the two bodies be such that they never experience a close approach (e.g., Lissauer, 1995). Given that we are in a regime of significant impact probability (see next paragraph), one can define an embryo size such that most satellitesimals dragging into its feeding zone will be accreted. Suppose we take a satellitesimal of mass $m_{1}$, which corresponds to a radius $\sim 3 \mathrm{~km}$. Such a satelli- 
tesimal has a very fast evolution timescale of $\sim 8$ years. We calculate the drag distance $l_{d}=\left(v_{\mathrm{d}} 2 \pi a / 3 \Omega\right)^{1 / 2}$ (with $v_{d}=$ $\left.a / \tau_{\text {gas }}\right)$ such that $2 l_{d}$ is the distance a satellitesimal will drag after one synodic period of two objects located at $\sim 15 R_{\mathrm{J}}$ a distance $l_{d}$ from each other. Then using the condition $s_{l}=l_{d}$ we calculate an embryo mass $m_{d} \sim 2.2 \times 10^{25} \mathrm{~g}$, which corresponds to an object size $\sim 1500 \mathrm{~km}$ and a feeding size of $s_{l} \sim 32000 \mathrm{~km}$. The time it took for the satellitesimal to cross this feeding zone (assuming that satellitesimal growth can be ignored; i.e., the embryo has cleared its feeding zone) was $\sim 0.22$ years (the synodic time of two objects separated by $\sim 32000 \mathrm{~km}$ at $15 R_{\mathrm{J}}$ ). If we increase the concentration of solids by a factor of 4 then the embryo size becomes $760 \mathrm{~km}$ (the smaller embryo size results from the longer satellitesimal orbital decay times in a disk with four times less gas). Thus, once an embryo has reached a size $\sim 1000 \mathrm{~km}$ its growth rate is controlled by the inward drift time of the characteristic size of the satellitesimals it accretes. If it mostly accretes kilometer-sized satellitesimals, the time between the embryo stage to full satellite is tens of years. On the other end, if most of its mass comes from the accretion of other embryos then the upper limit on the satellite accretion time scale is $10^{4}$ years.

There are two mechanisms that limit the efficiency of drift augmented accretion. First, resonant capture may prevent satellitesimals from reaching the embryo. This, however, may not be an issue for the inner disk (or for the outer disk, though for a different reason; see Sect. 5). The satellite embryo may not be massive enough to prevent the orbital decay of kilometer-sized objects given the gas surface densities of the inner disk (see Sect. 6.2 where we discuss the resonant capture of Hyperion by proto-Titan and the absence of a corresponding object for Ganymede). Second, the drifting satellitesimal may "horseshoe" around the embryo and avoid being captured by it. Kary et al. (1993) give impact probabilities for various mass ratios of the secondary to the primary as a function of the secondary's physical radius divided by its Hill radius. For a $1000-\mathrm{km}$ embryo with density $\rho_{s}=1.5 \mathrm{~g} \mathrm{~cm}^{-3}$ this ratio is $r_{s} / r_{H} \sim 0.1$. As the embryo grows to satellite size this ratio will decrease slightly (due to the slight increase in its density). On the other hand, its feeding zone will increase, and it will get more chances to capture any given satellitesimal. Hence, we expect that the capture efficiency will improve slightly as the embryo grows (though for sufficiently large embryo masses, such that the gas flow around the secondary is changed significantly or a gap is opened, the efficiency may again decrease). Given the criterion used to calculate the embryo size, we are in the gas regime of significant impact probability. Then from Kary et al. (1993, their Fig. 9) we see that the impact probability for the case such that $r_{s} / r_{H}=$ 0.1 is $>0.6$ (in the limit that gas drag effectively damps eccentricities and inclinations of the feeder population; note that $m_{d} / M_{J} \sim 10^{-5}$ ). This impact probability is high enough that a minimum subnebula model may apply to the accretion of satellites (in contrast, giant planet cores have much smaller ratios of the physical size to their Hill radius and are therefore unable to efficiently capture inwardly drifting planetesimals). This is a significant result. It says that $\sim 1000-\mathrm{km}$ satellite embryos are effective barriers and will capture most inwardly migrating satellitesimals, thus limiting the amount of material that is allowed to spiral into the planet or inner embryos.

In order to obtain an embryo size that becomes an effective barrier for inwardly drifting satellitesimals a typical satellitesimal mass $m_{1}$ was used. We now attempt to provide further justification for this choice. As we mentioned before, one expects the dust and rubble scale-height eventually to decrease due to the effects of coagulation and turbulence decay. We consider a case such that the coagulation of dust produces particles that decouple from the gas in a time scale shorter than $10^{3}$ years, starting at a time when the disk cooled enough for water-ice condensation to take place in the outer region of the inner disk. First, we find the characteristic particle size that will decouple from the gas. Given our nebula parameters and using the condition $\Omega t_{s} \sim 1$, we obtain a particle size $\sim 50 \mathrm{~m}$ in the region between 15 and $20 R_{\mathrm{J}}$. This particle settles to the midplane and drifts in due to gas drag. As it does, it grows by sweep-up of smaller particles. Assuming a self-similar power law distribution as in Weidenschilling (1997), we use particles of size $\sim 10 \mathrm{~m}$ as the feeder population where most of the mass resides. We calculate the scale-height for these particles under strongly turbulent conditions $\left(\alpha \sim 10^{-4}-10^{-3}\right)$ using Eq. (18) and obtain $H_{p} \sim 0.04 H$ (head-wind decreases for $H_{p}<0.01 H$ such that the particle layer density $\bar{\rho}_{p}>\rho_{g}$ ). For weak turbulence $\left(\alpha=10^{-6}-10^{-5}\right)$ the scale-height for the same feeder particle would be about an order of magnitude smaller.

We can now calculate the time it takes for a $50-\mathrm{m}$ particle at $20 R_{\mathrm{J}}$ to evolve to $15 R_{\mathrm{J}}$ and the size it will grow to by the time it gets there. The size of the satellitesimal is given by solving

$$
\frac{d r_{p}}{d t}=\frac{\sum_{s} c}{8 \rho_{s} \beta a}=\frac{g_{1}(a, t)}{a},
$$

where $\beta=H_{p} / H$, and the initial size of the satellitesimals is taken to be the size of the object such that $\Omega t_{s} \sim 1$. The drift velocity of this satellitesimal is

$$
v_{d}=\frac{d a}{d t}=-\frac{3 C_{D} \Sigma c^{3}}{8 \rho_{s} G M_{P}} \frac{a}{r_{p}}=\frac{g_{2}(a, t)}{r_{p}} .
$$

We can decouple these equations by letting $r_{p}=g_{2} /(d a / d t)$ and plugging this equation into Eq. (19). For the inner disk, the temperature varies like $1 / r$ (which implies $g_{1}, g_{2} \propto$ $a^{-3 / 2}$ ) so that assuming solar mixtures the position of the satellitesimal as a function of time is given by

$$
\frac{d^{2} a}{d t^{2}}=\frac{1}{a}\left[\frac{a^{2}}{75 C_{D} \beta H^{2}}-\frac{3}{2}\right]\left(\frac{d a}{d t}\right)^{2}=\frac{1}{a} \Lambda\left(\frac{d a}{d t}\right)^{2} .
$$


Assuming that $\beta$ is constant then $\Lambda$ is constant and we can solve this equation exactly to give the particle velocity as a function of time

$$
v(t)=v_{0}\left[1+(\Lambda-1) \frac{\left|v_{0}\right|}{a_{0}} t\right]^{-\Lambda /(\Lambda-1)},
$$

where $v_{0}$ is the initial velocity of a satellitesimal of initial size $r_{p 0}$. We can now integrate this equation for our subnebula model to give an equation for time as a function of position

$$
t_{G}=\frac{a_{0}}{\left|v_{0}\right|(\Lambda-1)}\left[\left(\frac{a_{0}}{a}\right)^{\Lambda-1}-1\right]
$$

and finally the growth of the satellitesimal as a function of time

$$
r_{p}(t)=r_{p 0}\left[1+(\Lambda-1) \frac{\left|v_{0}\right|}{a_{0}} t\right]^{(\Lambda+3 / 2) /(\Lambda-1)} .
$$

We find that for the case of strong turbulence a $50-\mathrm{m}$ particle will take $t_{G} \sim 0.2$ years to drift between 20 and $15 R_{\mathrm{J}}$ and it will grow to a size of $\sim 1 \mathrm{~km}$, which may be a bit small to be efficiently accreted by an embryo. However, for the case of weak turbulence growth occurs much more quickly. In this case, the same particle grows to a size of $\sim 10 \mathrm{~km}$ in $\sim 0.2$ years after $\sim 1 R_{\mathrm{J}}$ of inward migration. Once satellitesimals have attained this size they are likely to get picked up by embryos inside of their own orbit. While this model is quite sensitive to the particle sizes chosen, it does point out that particles that decouple from the gas and drift in are subject to growth. This growth may be fast enough that most of these particles will become satellitesimals with sizes $>1 \mathrm{~km}$ after only a few $R_{\mathrm{J}}$ of inward migration, at which point they will slow down and eventually get captured by a satellite embryo inside their own orbit. Although uncertain, this calculation is roughly consistent with the gas-free calculation of characteristic satellitesimal sizes. This again points out that the minimum mass model may indeed provide a fair estimate of the mass of condensables initially present in the gas disk.

\section{Slow formation of Callisto}

Our model has Callisto forming from an extended, low optical depth gas disk. We expect this gas disk to be largely quiescent with very low gas viscosity. This means that the dust and rubble layer will quickly settle down to the midplane within a scale-height much smaller than the gas scaleheight. The size of the dust and rubble layer is determined by shear turbulence close to the midplane (Cuzzi et al., 1993).

First we calculate characteristic masses and lengths in analogy to the gas free planetary accretion problem (Hourigan and Ward, 1984) for the case in which the outer disk contains $\sim M_{\text {Callisto }}$ of solids. At $\sim 50 R_{\mathrm{J}}$ with $\Sigma_{s} \sim 53 \mathrm{~g}$ $\mathrm{cm}^{-2}$ and $\rho_{s}=1.5 \mathrm{~g} \mathrm{~cm}^{-3}$, we obtain $m_{1} \sim 2.9 \times 10^{14} \mathrm{~g}$, which corresponds to a satellitesimal radius of $\sim 0.36 \mathrm{~km}$ and $l_{1} \sim 13 \mathrm{~km}$. The embryo size is $m_{2} \sim 3.3 \times 10^{23} \mathrm{~g}$, which corresponds to a embryo radius of $\sim 370 \mathrm{~km}$ and $l_{2} \sim$ $1.4 \times 10^{4} \mathrm{~km}$.

If we perform the same calculations further out at $\sim 100 R_{\mathrm{J}}$ with $\Sigma_{s} \sim 26 \mathrm{~g} \mathrm{~cm}^{-2}$, we obtain $m_{1} \sim 2.3 \times 10^{15}$ $\mathrm{g}$, which corresponds to a satellitesimal radius of $\sim 0.72 \mathrm{~km}$ and $l_{1} \sim 53 \mathrm{~km}$. The embryo size is $m_{2} \sim 9.2 \times 10^{23} \mathrm{~g}$, which corresponds to an embryo radius of $\sim 530 \mathrm{~km}$ and $l_{2}$ $\sim 3.9 \times 10^{4} \mathrm{~km}$. These characteristic masses are about an order of magnitude smaller than the masses we obtained at $15 R_{\mathrm{J}}$ for $m_{2}$ and two orders of magnitude smaller for $m_{1}$.

Given the small scale-height of the particle layer, similar-sized satellite embryos will form quickly. In this regime, it may not be a bad assumption to consider growth time scales for a case such that the solids in the disk are in the form of satellitesimals. For the outer disk the Safronov accretion time is given by

$$
\begin{aligned}
\tau_{\mathrm{acc}} \sim & 10^{5}\left(\frac{\rho_{s}}{1 \mathrm{~g} \mathrm{~cm}^{-3}}\right)\left(\frac{r_{s}}{100 \mathrm{~km}}\right)\left(\frac{10 \mathrm{~g} \mathrm{~cm}^{-2}}{\Sigma_{s}}\right) \\
& \times\left(\frac{a}{150 R_{J}}\right)^{3 / 2} F_{g}^{-1} \text { years. }
\end{aligned}
$$

Using a density of solids in the outer disk $\Sigma_{s} \sim 10 \mathrm{~g} \mathrm{~cm}^{-2}$ and enhancement factor $F_{g} \sim O(1)$, this formula predicts that an embryo with $\rho_{s}=1.5 \mathrm{~g} \mathrm{~cm}^{-3}$ and size $\sim 500 \mathrm{~km}$ would be formed in $\sim 10^{6}$ years at $150 R_{\mathrm{J}}$. Several factors can alter this growth time scale. Larger values of the enhancement factor can speed up the growth of large objects in the outer disk. However, even though the Hill radius of an embryo is much larger than its physical radius, the low density of embryos leads to infrequent collisions and velocity dispersions comparable to the escape velocity of the embryos (see Appendix A). As a result, the focusing factor is unlikely to be much llarger than one. On the other hand, embryo collisions do not necessarily lead to accretion. Glancing collisions may not lead to sticking or may yield embryo spins resulting in ejection of the colliding pair. Furthermore, collisional disruption of embryos $>100 \mathrm{~km}$ can decrease the efficiency of the growth process (see Appendix B). Finally, the above estimate was obtained assuming that all of Callisto was spread out to $150 R_{\mathrm{J}}$. Placing a fraction of Callisto's mass in the outer disk would lead to longer embryo growth times (but then Callisto may differentiate unless a source of solids from the Roche-lobe is considered). From this we conclude that embryos with sizes $\$ 500 \mathrm{~km}$ may be formed in a time scale of $\sim 10^{6}$ years at $\sim 100 R_{\mathrm{J}}$.

To find the characteristic sizes of outer disk embryos in the presence of gas drag we turn to drift augmented accretion. In the absence of global turbulence, we make the assumption that local turbulence due to the gas-dust shear layer close to the subnebula midplane will adjust itself to maintain rough parity such that $\bar{\rho}_{p} \approx \rho_{g}$ (Cuzzi et al., 1993). 
This approximate relation can be used to estimate $H_{p}$. Alternatively, we can write

$$
H_{p} \approx C_{T} \frac{\Delta v}{\Omega^{2} t_{s}}
$$

where $C_{T} \approx 0.01$ is a constant (Cuzzi et al., 1993). The scale height is $H_{p} \sim 0.01 H$ for particle of size $\sim 10 \mathrm{~cm}$. As we did in the case of Ganymede, we now calculate the sweep-up time for a particle with radius $r_{p}$. We find $\tau_{\text {sweep }} \sim 5.5\left(r_{p} / 1\right.$ $\mathrm{km})$ years at $50 R_{\mathrm{J}}$ and $\tau_{\text {sweep }} \sim 22\left(r_{p} / 1 \mathrm{~km}\right)$ years at $100 R_{\mathrm{J}}$. These growth time scales are $\sim 50$ times faster than the drift times for any given object size (see Fig. 5b).

The above sweep-up times do assume that the dust and rubble surface density stays constant. Once the sweep-up growth slows down due to dust and rubble depletion, continued embryo growth will depend on the drift augmented accretion of satellitesimals. As we did for the inner disk, we now ask what size embryo stands a significant chance of capturing satellitesimals of characteristic size $m_{1}$ drifting into its feeding zone. We choose the criterion $s_{l}=l_{d}$ as we did before and find the characteristic quantities $m_{d} \sim 2 \times$ $10^{24} \mathrm{~g}$, which corresponds to an embryo size of $690 \mathrm{~km}$ (for $\rho_{s}=1.5 \mathrm{~g} \mathrm{~cm}^{-3}$ ), and $s_{l} \sim 4.8 \times 10^{4} \mathrm{~km}$ at $50 R_{\mathrm{J}}$. At $100 R_{\mathrm{J}}$ we get $m_{d} \sim 1.2 \times 10^{24} \mathrm{~g}$, corresponding to a radius of 580 $\mathrm{km}$, and $s_{l} \sim 8 \times 10^{4} \mathrm{~km}$. A wrinkle results from the small scale-height and long drag times inherent in this problem. If we calculate how long it took satellitesimal $m_{1}$ to cross a distance $l_{d}$ we find $t_{d} \sim 3$ years at $50 R_{\mathrm{J}}$ and $t_{d} \sim 10$ years at $100 R_{\mathrm{J}}$. These time scales are sufficiently long to allow some satellitesimal growth during the time it takes to cross the embryo's feeding zone. Taking this effect into account the corrected embryo sizes turn out to be slightly smaller $590 \mathrm{~km}$ at $50 R_{\mathrm{J}}$ and $540 \mathrm{~km}$ at $100 R_{\mathrm{J}}$.

The first thing to notice is that the smaller embryo mass now corresponds to the larger semi-major axis. This is because the larger value for $m_{1}$ at $100 R_{\mathrm{J}}$ led to longer time scales to cross the feeding zone, thus requiring a smaller embryo size to satisfy our capture condition. Even though the smaller embryo size now occurs further out, the decrease in size is not sufficient to compensate for the decrease in gas surface density. The result is that inner embryos will drag in first and drift augmented growth will stop (the small scaleheight means that all the dust and rubble will quickly become depleted). Thus, in our model $\sim 500 \mathrm{~km}$ represents the characteristic size that will form before drifting in to Callisto's radial location. This characteristic size decreases significantly if one considers disks of higher solid concentration (higher solid concentration disks may be desirable for several reasons; see also Paper II). For instance, if we keep the surface density of solids constant but decrease the gas density by a factor of 4 we obtain $m_{d} \sim 1.4 \times 10^{23} \mathrm{~g}$, which corresponds to a size of $\sim 280 \mathrm{~km}$ at $100 R_{\mathrm{J}}$ (taking into account satellitesimal growth).

As before, we need to address the issue of the capture efficiency. For the outer disk, the gas density is too low and the mass of the satellitesimals too high to avoid being captured into resonances, if initially placed in low eccentricity orbits. However, proto-Callisto may not have captured objects into resonance because the typical (for embryo sizes in the range 100-500 km) embryo eccentricities near Callisto's orbital location are $e \sim 0.02-0.07$, where we have assumed that the random velocities are on the same order as their escape velocities (Appendix A). These eccentricities are similar or larger than the critical eccentricity for which capture probability sharply drops off: $e_{\text {crit }} \sim \eta \sim$ 0.03 (Malhotra, 1993). Hence, for low gas surface densities typical satellitesimal eccentricities may again lead to low probability of resonance capture.

Having argued that resonant capture is unlikely to take place, we ask what fraction of the population of objects that drift into Callisto's feeding zone will be accreted by it. In the case of Callisto, the synodic time scale is much shorter than the drift time across the feeding zone of the population of feeders. In this weak gas regime, the accretion efficiency is limited by the inclinations of the drifting satellitesimals. Typical inclinations for such objects may be smaller than their eccentricities (see Appendix A). In the neighborhood of Callisto $i_{H}=i a / H \sim 0.06-0.26$ for embryos in the range $100-500 \mathrm{~km}$. Given that for Callisto $r_{s} / r_{H}=0.048$, we can use the simulations in Kary et al. (1993), their Fig. 12 to estimate the accretion probability at $>0.7$. Hence Callisto may capture most of the satellitesimals that drift into its feeding zone (this argument also applies to proto-Callisto nearly unchanged). It is interesting to compare the vertical extent of the satellitesimals $H_{s}$ to the feeding zone of Callisto $\sim 2 r_{H} \sim 1.4 R_{\mathrm{J}}$. From Appendix A, we see that equilibrium sets a satellitesimal velocity dispersion $u \sim v_{\text {esc }}$, where $v_{\text {esc }}$ is the escape velocity for such a satellitesimal. Then we can use $H_{s} \sim u / \Omega$ to get $H_{s} \sim 0.3 R_{\mathrm{J}}$ for $100-\mathrm{km}$ satellitesimals at $30 R_{\mathrm{J}}$, and $H_{s} \sim 1.3 R_{\mathrm{J}}$ for $500-\mathrm{km}$ satellitesimals at $30 R_{\mathrm{J}}$ (for comparison $H \sim 4 R_{\mathrm{J}}$ at $30 R_{\mathrm{J}}$ ).

We can now calculate the time it takes to accrete Callisto by the time it takes gas drag to clear the outer disk of such embryos. Such a calculation yields an accretion time scale for Callisto of $10^{5}-10^{6}$ years. This is calculated under the conservative condition that the gas density in the outer disk does not decrease over time. Since the actual concentration of solids is likely to be somewhat larger than given by cosmic mixtures (see Paper II), we give $\sim 10^{6}$ years as the clearing time for the outer disk. For instance, a concentration factor of 4 would lead to embryo sizes $\sim 300 \mathrm{~km}$, which would take $2 \times 10^{6}$ years to drift in from $150 R_{\mathrm{J}}$ to Callisto's radial location (this time was obtained by integrating Eq. (20) with $\rho_{s}=1.5 \mathrm{~g} \mathrm{~cm}^{-3}$ ). This time scale is significantly shorter than the gas dissipation time scale (taken to be the photoevaporation timescale $\sim 10^{7}$ years (Shu et al., 1993)), so no embryos would be stranded outside of Callisto.

The upshot is that Callisto's accretion time scale differs significantly from that of Ganymede because Callisto draws materials from much further out $\left(\sim 150 R_{\mathrm{J}}\right.$, compared to $23 R_{\mathrm{J}}$ for Ganymede). To complete its accretion it must 
a

a $\quad a=20 R_{s}, T=100 \mathrm{~K}$

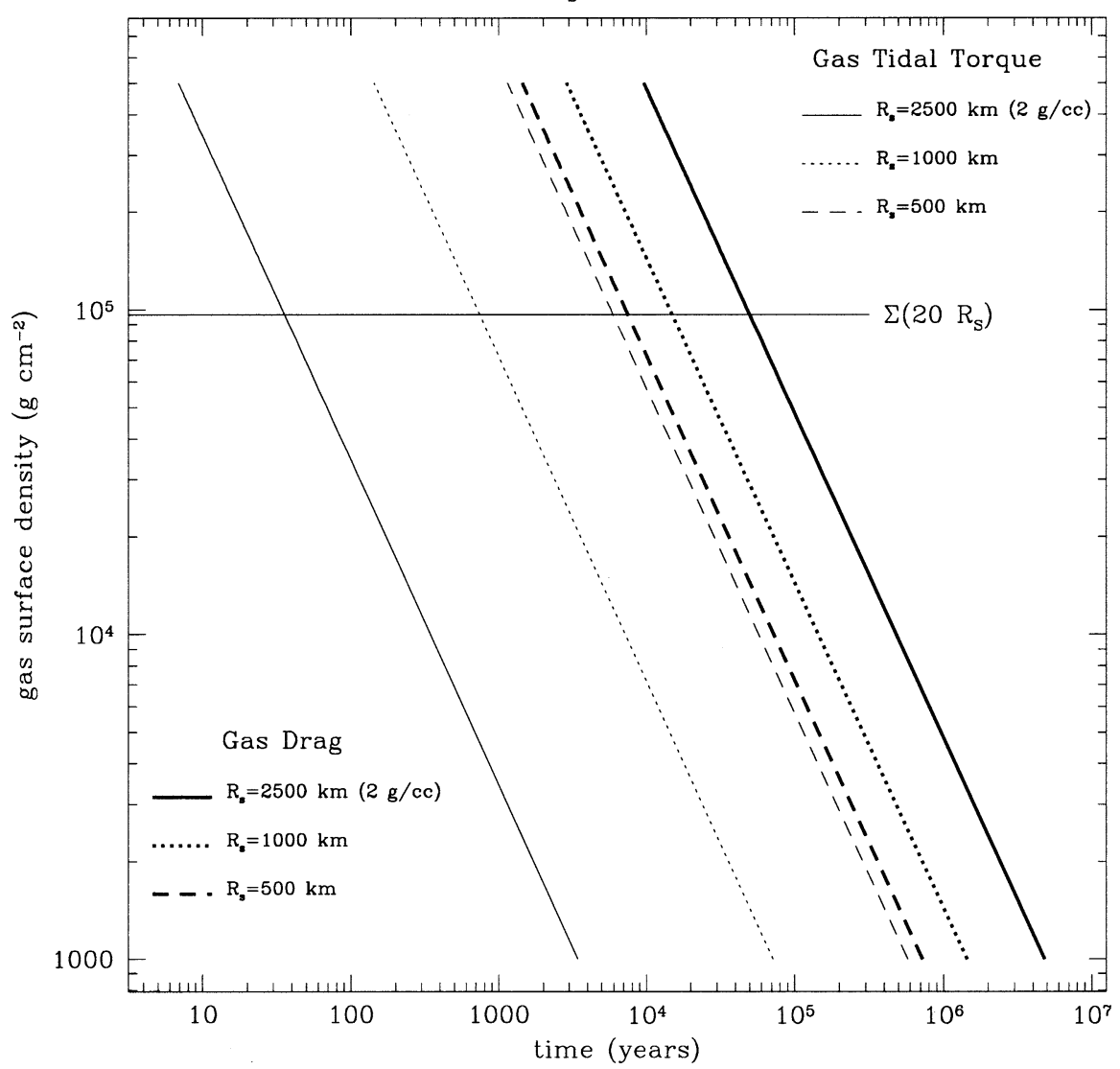

Fig. 6. (a) Orbital decay times of satellitesimals of various sizes due to gas drag and gas tidal torque at $20 R_{\mathrm{S}}$. Unless otherwise indicated the density is $\rho_{\mathrm{s}}$ $=1.5 \mathrm{~g} \mathrm{~cm}^{-3}$. The gas surface density for our models is indicated in the plot. The decay times of smaller objects are dominated by gas drag, while larger objects are controlled by gas tidal torque, with the transition occuring between 500 and $1000 \mathrm{~km}$. (b) Orbital decay times of satellitesimals of various sizes due to gas drag and gas tidal torque at $70 R_{\mathrm{J}}$. Unless otherwise indicated the density is $\rho_{\mathrm{s}}=1.5 \mathrm{~g} \mathrm{~cm}^{-3}$. The gas surface density for our models is indicated in the plot.

contend with the drag times of embryos. Since the gas density is much lower in the outer disk, the distances larger, and the dynamical times longer, the resulting accretion time scale for Callisto accretion will be much longer $\left(\sim 10^{6}\right.$ years) than it was for Ganymede $\left(\sim 10^{3}-10^{4}\right.$ years).

It is natural to ask how sensitive these calculations are to the size of the disk. It turns out that the characteristic size of the embryos does not change much with disk size. This is because of the countering effects of the increase in Hill radius with semi-major axis and the decrease in surface density. A smaller disk size would yield similar embryo sizes, which would take a slightly shorter time to evolve to Callisto. A larger disk can also be considered, but in that case one would need to explain why the irregular satellites were not dragged into the planet.

\section{Saturn's regular satellite system}

In order to apply our model to Saturn we first need to constrain the nebula parameters for Saturn as we did for
Jupiter. First, we note that the ratio of the reconstituted Galilean satellite masses to the saturnian satellite masses is $\approx 3.7$. On the other hand, the ratio of the atmospheric envelopes of Jupiter to Saturn is $\approx 3.7$ for giant planet core masses of $\approx 12$ Earth masses, consistent with nominal values.

In Fig. 2, we plot two models. The first model simply assumes the same mass ratio $(\sim 3.7)$ with respect to the jovian satellite system applies to both the inner and the outer disks. The second model puts just enough mass between 60 and $200 R_{\mathrm{S}}$ to make Iapetus (since Iapetus is made of ice, the minimum mass model requires a subnebula of $\sim 200 M_{\text {Iapetus }}$ ).

We get a disk size of $\sim 220 R_{\mathrm{S}}$ by scaling the outer disk of Jupiter $\left(\sim 150 R_{\mathrm{J}}\right)$ by the ratio of the Hill radii of Saturn and Jupiter. It is encouraging to note that Phoebe is located at $215 R_{\mathrm{J}}$. This object has a retrograde orbit of high inclination and eccentricity, leaving little doubt that it was captured. Therefore, we adjust the size of Saturn's outer disk slightly to $\sim 200 R_{\mathrm{S}}$ to fit in with the location of Phoebe. As was the case with the irregular satellites of Jupiter, we use gas drag to explain the absence of captured objects inside 


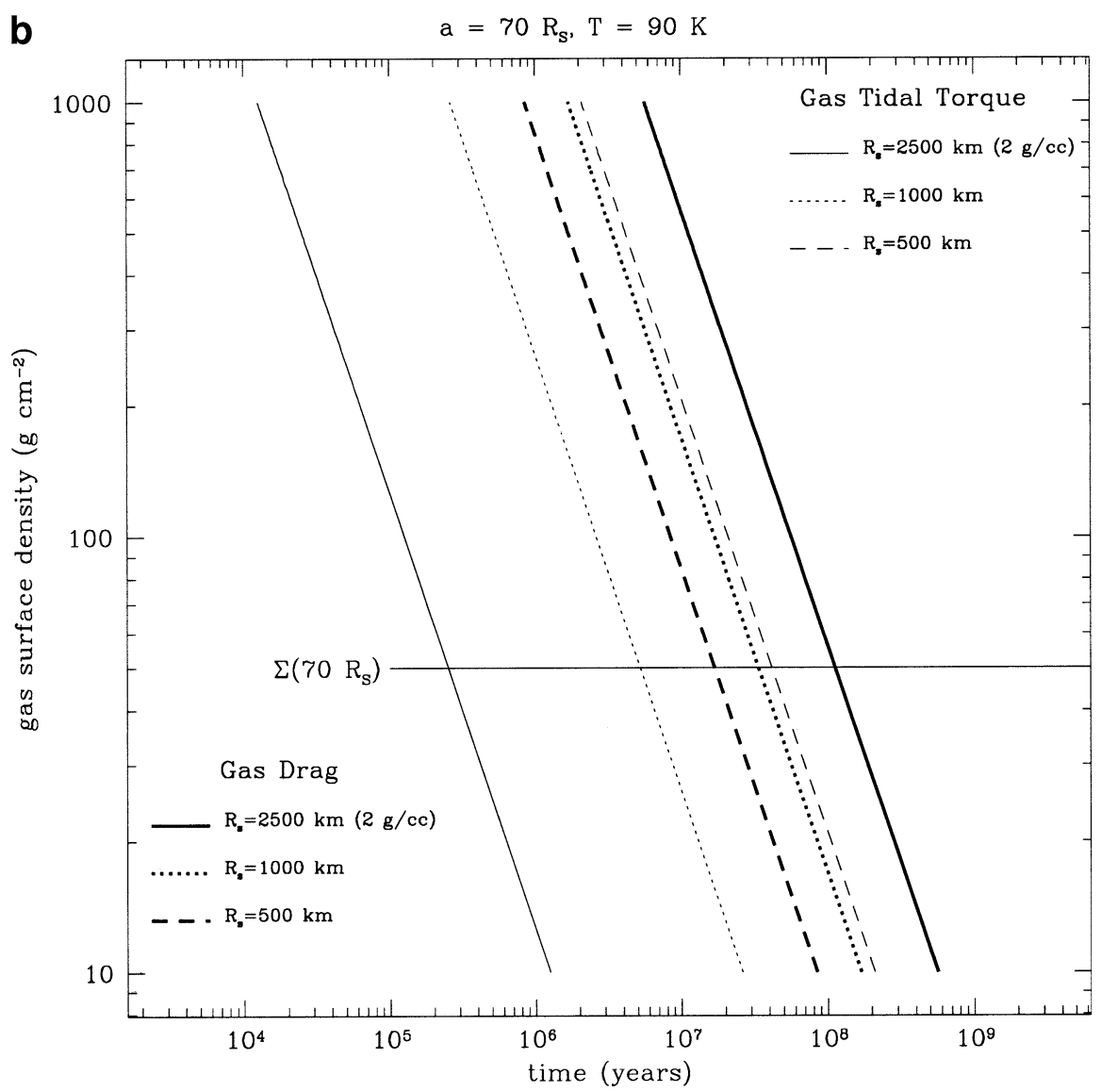

Fig. 6 (continued)

the orbit of Phoebe (a couple of small irregulars are located just inside of Phoebe).

To constrain the temperature of the saturnian subnebula, we assume that the accretion of methane ice explains the methane in Titan's atmosphere (Lewis, 1972). That sets a temperature of $\approx 100 \mathrm{~K}$ at $20 R_{\mathrm{S}}$. Inside this location the subnebula is taken to be optically thick and the temperature to vary inversely with radius. In Fig. 3, we have plotted the subnebula temperature as a function of distance in units of the planetary radius.

As we did before in the case of Jupiter, we calculate characteristic masses based on the gas-free planetary accretion model. In Saturn's inner disk at $\sim 20 R_{\mathrm{S}}$ with $\Sigma_{s} \sim 900 \mathrm{~g}$ $\mathrm{cm}^{-2}$ and $\rho_{s}=1.5 \mathrm{~g} \mathrm{~cm}^{-3}$, we obtain $m_{1} \sim 2.9 \times 10^{16} \mathrm{~g}$, which corresponds to a satellitesimal radius of $\sim 1.7 \mathrm{~km}$ and $l_{1} \sim 30 \mathrm{~km}$. The embryo size is $m_{2} \sim 1.8 \times 10^{24} \mathrm{~g}$, which corresponds to a embryo radius of $\sim 660 \mathrm{~km}$ and $l_{2} \sim 1.2 \times$ $10^{4} \mathrm{~km}$.

In Fig. 6a, we plot the orbital decay times at $20 R_{\mathrm{S}}$ as a function of the surface gas density for several particle sizes. As one would expect, these evolution times are generally longer than those we calculated for the case of Ganymede. The reason is that the orbital period at Titan is longer than the orbital period at Ganymede. If we add to this the fact that the surface density of Saturn's less massive and more spread-out (due to Saturn's larger Hill radius) disk is considerably lower than that of Jupiter's disk (see Fig. 2), we find that the evolution times in Saturn's disk are generally about an order of magnitude longer than the corresponding evolution times (for a similar-sized object) in Jupiter's disk. It is instructive to write the drag times at Saturn as

$$
\begin{aligned}
\tau_{\text {gas }} \approx & 13\left(\frac{\rho_{s}}{1 \mathrm{~g} \mathrm{~cm}^{-3}}\right)\left(\frac{r_{p}}{1 \mathrm{~km}}\right)\left(\frac{90 \mathrm{~K}}{T}\right)^{3 / 2} \\
& \times\left(\frac{10^{5} \mathrm{~g} \mathrm{~cm}^{-2}}{\Sigma}\right) \text { years. }
\end{aligned}
$$

Now we turn to the process of satellite formation. As before, first we build up embryos using a sweep-up of dust and rubble. The time scale for this process at Saturn is

$$
\begin{aligned}
\tau_{\text {sweep }} \approx & 0.57\left(\frac{\rho_{s}}{1 \mathrm{~g} \mathrm{~cm}^{-3}}\right)\left(\frac{r_{p}}{1 \mathrm{~km}}\right)\left(\frac{a}{R_{S}}\right)\left(\frac{90 \mathrm{~K}}{T}\right)^{1 / 2} \\
& \times\left(\frac{10^{5} \mathrm{~g} \mathrm{~cm}^{-2}}{\Sigma}\right)\left(\frac{H_{p}}{H}\right) \text { years. }
\end{aligned}
$$

We see that making a $1000-\mathrm{km}$ embryo would take $\sim 10^{4} \mathrm{H}_{p} / \mathrm{H}$ years at Titan's location, or about 10 times 
longer than it took at Ganymede. Allowing $H_{p}<H$, the sweep-up time scale is always shorter than the drag time scale for $a<22 R_{\mathrm{S}}$. Beyond this distance our model has an optically thin, quiescent disk with $H_{p} \ll H$. Therefore, in general, $\tau_{\text {sweep }}<\tau_{\text {gas }}$. In the inner disk (inside of $20 R_{\mathrm{S}}, T=$ $100 \mathrm{~K}$ ), where the temperature is inversely proportional to the radial location, the ratio of the sweep-up time to the gas drag time is independent of semi-major axis and particle size $\tau_{\text {sweep }} / \tau_{\text {gas }} \approx H_{p} / H$. Since the sweep-up times for Saturn are about an order of magnitude longer than for Jupiter, for Saturn there is an increased likelihood that bombardment from outside the Roche-lobe disrupted embryo growth. Furthermore, characteristic embryo sizes $<1000$ $\mathrm{km}$ are significantly smaller for Saturn's disk. It seems likely that more embryos were lost in the case of Saturn, which might explain the absence of large satellites inside Titan's orbital radius where collisional events might have been energetic enough to break up satellite embryos (Greenberg et al., 1977). It must be mentioned, however, that weaker turbulence in Saturn's disk may reduce the value of $H_{p} / H$ and alter this conclusion.

Now we calculate the size of an embryo that will be effective at capturing satellitesimals of mass $m_{1}$ drifting across its feeding zone. At Titan we find $m_{d} \sim 3.5 \times 10^{24}$, which corresponds to $\sim 820 \mathrm{~km}$ (compared to $\sim 1500 \mathrm{~km}$ at Ganymede) and a feeding zone of $l_{d} \sim 2.9 \times 10^{4} \mathrm{~km}$. It took an $m_{1}$ satellitesimal 0.6 years (compared to 0.22 years at Ganymede) to cross the embryo's feeding zone. For $H_{p}=$ $H$, it will take $1.3 \times 10^{4}$ years to build an embryo of size $\sim 820 \mathrm{~km}$ (compared with $\sim 2700$ years at Ganymede to form an embryo of size $\sim 1500 \mathrm{~km}$ ). Decreasing the gas density by a factor of 4 leads to an embryo size of $\sim 410 \mathrm{~km}$ (compared to $760 \mathrm{~km}$ for the same concentration factor for Ganymede). Such small embryos may be vulnerable to break-up by hypervelocity impacts.

Given that the satellite formation time is the embryo accretion time plus the drift accretion time of embryos, we obtain a time of $10^{4}-10^{5}$ years for Titan's accretion (compared with $10^{3}-10^{4}$ years for Ganymede's accretion).

\subsection{Formation of Iapetus}

We view Iapetus as an analog of Callisto in the sense that it formed on a long timescale from the materials in the optically thin outer disk. Aside from its size, there is one way, however, in which Iapetus is quite different from Callisto, namely, its low density $\sim 1.03 \pm 0.1 \mathrm{~g} \mathrm{~cm}^{-3}$ (Dermott and Thomas, 1994). It is likely that direct accretion from materials in the solar nebula would have led to larger density for this object. We speculate that the reason for the low density of Iapetus is that for the low gas density of Saturn's envelope the source of the material in Saturn's outer disk came from the ablation of water and methane ice of infalling planetesimals, because at the edge of the planetary envelope refractories were lost as a result of dust settling, or because of the preferential break-up of icy ob- jects far from the planet. It is also possible that material was derived from the ablation of planetesimals passing through the circumplanetary disk after its formation. We note that spectrally Iapetus is most like Hyperion (Owen et al., 2001), which our model has forming in the transition region between the inner and the outer disks (see Sect. 6.2). It is also worth noting that Phoebe's visual spectral properties are unlike those of Hyperion or Iapetus (e.g., Buratti et al., 2002).

The time scale for formation of Iapetus varies significantly depending on the specific model chosen for the surface density in Saturn's outer disk. For the sake of specificity, here we choose a model that yields enough condensables for one Iapetus mass between 60 and $200 R_{\mathrm{S}}$ (see Fig. 2). This results in a minimum mass subnebula model such that $\sim 200 M_{\text {Iapetus }}$ is in the outer disk. We first calculate the characteristic masses (for the dashed curve in Fig. 2, Iapetus model). In Saturn's outer disk at $\sim 70 R_{\mathrm{S}}$ with $\Sigma_{s} \sim 1.3 \mathrm{~g} \mathrm{~cm}^{-2}$ and $\rho_{s}=1 \mathrm{~g} \mathrm{~cm}^{-3}$ (which is the density of Iapetus), we obtain $m_{1} \sim 1.3 \times 10^{11} \mathrm{~g}$, which corresponds to a satellitesimal radius of $\sim 0.03 \mathrm{~km}$ and $l_{1} \sim 1.8$ $\mathrm{km}$. The embryo size is $m_{2} \sim 3.7 \times 10^{21} \mathrm{~g}$, which corresponds to a embryo radius of $\sim 96 \mathrm{~km}$ and $l_{2} \sim 5.5 \times 10^{3}$ $\mathrm{km}$.

At this location the size of an embryo that will capture a significant fraction of the satellitesimals of mass $m_{1}$ that drift by is $m_{d} \sim 1.6 \times 10^{24} \mathrm{~g}$, which corresponds to a radius of $\sim 720 \mathrm{~km}$ and $l_{d} \sim 7.8 \times 10^{4} \mathrm{~km}$. As was the case with Callisto, however, the satellitesimal will grow due to rubble and dust sweep-up as it crosses the embryo's feeding zone (assuming $\bar{\rho}_{p} \approx \rho_{g}$ ). Therefore, the above estimates need to be corrected to take into account that the drift times increase as the satellitesimal grows. Then we obtain $m_{d} \sim 1.2 \times 10^{24}$ $\mathrm{g}$, which corresponds to a radius of $\sim 670 \mathrm{~km}$ and $l_{d} \sim 7.2$ $\times 10^{4} \mathrm{~km}$. Assuming $\bar{\rho}_{p} \approx \rho_{g}$, it will take about $3.4 \times 10^{5}$ years to grow an embryo of that size by dust and rubble sweep-up at that location. It is interesting to note that $m_{2} \ll$ $m_{d} \lesssim M_{\text {Iapetus. }}$. A model with four times less gas density leads to an embryo size of $\sim 350 \mathrm{~km}$ at Iapetus.

To complete the accretion of Iapetus we need to accumulate several embryos. Since the time scale for drift of a $500-\mathrm{km}$ embryo at Iapetus' location is $10^{6}-10^{7}$ years (see Fig. $6 \mathrm{~b}$ ), we take this value to be the formation time scale for Iapetus.

\subsection{Formation of Hyperion}

The origin of Hyperion in the 4:3 mean-motion resonance with Titan presents a significant challenge. A tidal origin of resonance capture as may apply to Galilean satellites (Malhotra and Dermott, 1990) seems unlikely to apply to the case of Titan and Hyperion. Given Titan's size and distance from Saturn, significant expansion of its orbit would require Saturn's dissipation parameter $Q$ to be much lower than the lower limit set by the proximity of Mimas. Lee and Peale (2000) concluded that Hyperion could have 
formed with its present orbital properties by the accretion of satellitesimals and subsequent capture into the 4:3 resonance provided that (a) there was a steep gradient $\left(\propto r^{-3}\right)$ of disk and particles densities, (b) Titan grew to its present size and eccentricity on a timescale of $10^{4}-10^{5}$ years, and (c) no particles were added to the outside of the disk. Given that our model puts Hyperion in the transition region of the gaseous disk, where one would naturally expect steep density gradients (i.e., the steep density gradient would result from the edge of the inner disk, not from global disk properties), we find that Lee and Peale's model requirements agree very well with our satellite formation scenario. We note that these authors found gas drag to be necessary in order to induce satellitesimal orbital decay and capture into resonance.

It might appear that condition (c) is inconsistent with our model but we argue otherwise. Particles from the outer, low density disk would start arriving well after Hyperion's accretion was already complete and would be composed of a sparse population of rather sizeable objects (tens to hundreds of kilometers) with significant eccentricities and inclinations (thus reducing the probability of resonant capture).

We now check to see whether proto-Titan may capture satellitesimals into resonance in the gas regime corresponding to its accretion. The threshold mass such that protoTitan can halt the inward migration of a satellitesimal in the vicinity of a $j+1: j$ resonance is given by (Malhotra, 1993)

$$
\mu_{T H}=\left(\frac{M_{S}}{M_{P}}\right)_{T H}=\frac{2}{C_{\mathrm{ad}}} \frac{a}{j(j+1)}\left(\eta v_{K} \tau_{\mathrm{gas}}\right)^{-1},
$$

where $M_{S}$ is the mass of the satellite, $M_{P}$ is the mass of the planet, and $C_{\mathrm{ad}}=C_{\mathrm{ad}}^{\prime} /\left(1+\eta / e^{*}\right) \lesssim 3.3$ is a numerical constant. As in Malhotra (1993), we make the assumption that in the strongly damped regime the quantity $\left(1+\eta / e^{*}\right)$ is roughly constant with forced eccentricity $e^{*} \sim \eta$. In terms of our model parameters, the threshold mass can be written as

$$
\begin{aligned}
\mu_{T H} \simeq & \frac{2.9 \times 10^{-3}}{j(j+1)}\left(\frac{1 \mathrm{~g} \mathrm{~cm}^{-3}}{\rho_{s}}\right)\left(\frac{a}{R_{S}}\right)^{1 / 2}\left(\frac{T}{90 \mathrm{~K}}\right)^{1 / 2} \\
& \times\left(\frac{1 \mathrm{~km}}{r_{p}}\right)\left(\frac{\Sigma}{10^{5} \mathrm{~g} \mathrm{~cm}^{-2}}\right),
\end{aligned}
$$

which yields $\mu_{T H} \simeq 3.5 \times 10^{-4}$ for a $1-\mathrm{km}$ object for the 4:3 resonance with Titan located at Hyperion $\left(24.5 R_{\mathrm{S}}\right)$, which is near the inner edge of the transition region $\left(\mu_{\mathrm{Titan}}\right.$ $=2.4 \times 10^{-4}$ ). It is possible that Titan migrated inward over its history. This would place the 4:3 resonance in regions of lower gas density, thus permitting even lower threshold masses. Allowing for a factor of 3-4 less gas also makes it easier for proto-Titan to capture satellitesimals into resonance.
For Jupiter we can write

$$
\begin{aligned}
\mu_{T H} \simeq & \frac{2.3 \times 10^{-3}}{j(j+1)}\left(\frac{1 \mathrm{~g} \mathrm{~cm}^{-3}}{\rho_{s}}\right)\left(\frac{a}{R_{J}}\right)^{1 / 2}\left(\frac{T}{130 \mathrm{~K}}\right)^{1 / 2} \\
& \times\left(\frac{1 \mathrm{~km}}{r_{p}}\right)\left(\frac{\Sigma}{10^{5} \mathrm{~g} \mathrm{~cm}^{-2}}\right),
\end{aligned}
$$

which yields $\mu_{T H} \simeq 1.6 \times 10^{-3}$ for a $1-\mathrm{km}$ object for the $4: 3$ resonance with Ganymede located at $18 R_{\mathrm{J}}\left(\mu_{\text {Ganymede }}=\right.$ $7.8 \times 10^{-5}$ ). This may seem to indicate that Ganymede did not capture a satellite in resonance because it was not massive enough to stop the inward drag of satellitesimals outside its orbit; however, there is also the possibility of capturing a larger satellitesimal into resonance. Lee and Peale (2000) argue that accretion does not generally proceed within resonances because satellitesimal interactions may scatter captured satellitesimals out of the resonance. In their model Hyperion forms outside of resonance and then drifts and gets captured into resonance. Given Jupiter's nebula parameters, a full-sized Ganymede ( $\mu_{\text {Ganymede }}$ ) cannot capture objects smaller than $\sim 100 \mathrm{~km}$, while the corresponding size for a full-sized Titan $\left(\mu_{\text {Titan }}\right)$ in Saturn's disk is $\sim 5 \mathrm{~km}$. Hence, it is still possible that Ganymede did cap ture such objects, which were subsequently lost due to collisions or unstable orbits. It is also possible that other effects prevented proto-Ganymede from capturing satellite embryos by gas drag (Hahn et al., 1995).

\subsection{Formation of satellites inside Titan}

The first thing to note about the satellites inside Titan is that by mass they constitute only $\sim 3 \%$ of the total mass of the satellite system. The second thing is that they have low densities (Tethys, Enceladus, and Mimas have densities consistent with almost all ice, while Rhea is mostly ice (see Table 1) with the exception of Dione $\left(\rho_{s} \approx 1.5 \mathrm{~g} \mathrm{~cm}^{-3}\right)$. The higher density for Dione may reflect a rockier composition or some endogenic process (as evidenced by the observed resurfacing on this satellite but not Rhea) such as cryovolcanism that can close "pores" and lead to larger bulk density. Other factors, such as under-dense ice and the effects of impacts, may mar a straightforward interpretation of satellite densities based on composition alone. Nevertheless, it is likely that as a group these satellites are mostly made of ice. It is also important to note that there is a large gap between the outermost satellite in this group (Rhea at $8.7 R_{\mathrm{S}}$ ) and Titan (at $20 R_{\mathrm{S}}$ ). Last, we note that all large saturnian satellites (with the exception of Iapetus) appear to be sorted according to size, with the smaller satellites further in. We consider this sequence to be significant. We will return to this issue in Paper II.

We expect that these satellites accreted in the presence of significantly less gas than did Titan $\left(\Sigma \sim 10^{4} \mathrm{~g} \mathrm{~cm}^{-2}\right.$; see Paper II) about $10^{5}$ years after the end of Saturn's accretion, 
once the planet cooled enough for water-ice condensation at the orbit of Rhea to take place. Despite their small size, given their drag times, we estimate it took $10^{4}-10^{5}$ years to accrete these objects.

\section{Uranian satellite system}

As we did in the case of Saturn, we begin our discussion of the satellite system of Uranus by comparing the ratio of masses of the atmospheric envelopes to the ratio of masses of the satellite systems. Assuming Saturn to have a $\sim 15$ Earth mass core and Uranus a smaller $\sim 10$ Earth mass core, the mass ratio of the envelopes for the two planets is $\sim 18$. While substantially uncertain, this value compares favorably with the mass ratio of the satellite systems of the two planets $\sim 15$.

All the regular satellites of Uranus are all well inside Uranus' centrifugal radius located at $\sim 57 R_{\mathrm{U}}$. The irregular satellites Caliban and Stephano are found at 280 and $309 R_{\mathrm{U}}$, or approximately $R_{\mathrm{H}} / 10$. Inside the centrifugal radius, the main satellites of Uranus are Oberon (at $22 R_{\mathrm{U}}$ ), Titania (at $17 R_{\mathrm{U}}$ ), Umbriel (at $10 R_{\mathrm{U}}$ ), Ariel (at $7 R_{\mathrm{U}}$ ), and Miranda (at $\left.4.9 R_{\mathrm{U}}\right)$. As before, it is more meaningful here to establish correspondences between satellites not in terms of planetary radii but in terms of the Hill radius of the planet (see Fig. 1). Scaled by the Hill radius of the primary, the location of Oberon corresponds closely to the location of Rhea in Saturn's satellite system. Moreover, the size of Oberon (760 $\mathrm{km})$ is quite similar to the size of Rhea $(764 \mathrm{~km})$. Furthermore, there is evidence in the uranian system of sorting according to size as there is in the saturnian system. The main difference between the two families of objects is that Saturn's inner satellites (satellites inside of Titan) are considerably less dense (presumably icier) than Uranus' regular satellites. In fact, of the uranian satellites, only Miranda has a density $\left(1.2 \mathrm{~g} \mathrm{~cm}^{-3}\right)$ consistent with an object made mostly of ice. It is indeed remarkable that here, too, there is evidence of endogenic activity.

It has been suggested that the satellites of Uranus may have been the by-product of the impact event that led to its present obliquity (e.g., Pollack et al., 1991). However, such a process is unlikely to lead to a sufficiently extended particle disk to produce satellites as far as $22 R_{\mathrm{U}}$ (Canup and Ward, 2000). Furthermore, the evidence for possible systematic increases in density and size (see Fig. 1) for these satellites is hard to reconcile with an isolated impact origin. Instead, we choose to follow the same general outline to form the satellites of Uranus as we applied to the formation of the regular satellites of Saturn and Jupiter.

A minimum mass model in which the gas disk extends out to the centrifugal radius of the planet at $57 R_{\mathrm{U}}$ would set the average gas density at $1.4 \times 10^{4} \mathrm{~g} \mathrm{~cm}^{-2}$, which corresponds to a pressure of $\sim 1.5 \times 10^{-3}$ bar at $22 R_{\mathrm{U}}$. This is close to the surface gas density at which the disk becomes optically thin (given gaseous opacity). The temperature gradient in the uranian subnebula may not have been sufficiently strong to drive turbulence. So we would expect a cool, largely quiescent disk with gas surface density of $\sim 10^{4} \mathrm{~g} \mathrm{~cm}^{-2}$ as the environment in which the uranian satellites accreted. This is similar to the environment we hypothesize led to the inner Saturnian moons (see also Paper II).

We interpret the gap between the location of the centrifugal radius and the outermost satellite as suggestive of significant satellite migration. These objects may have taken $\sim 10^{5}$ years to accrete.

\section{Starved disk model}

A scenario in which the giant planet satellites accrete from a disk produced by the direct infall of gas and solids from heliocentric orbit, leading to long satellite assembly times $\sim 10^{6}$ years despite the short disk accumulation times $\sim 10^{2}$ years (Stevenson, personal communication), has numerous issues to overcome. Here we mention some of the outstanding ones and leave development of such a model (if viable) for later work.

First is the issue of satellite survival. Recent numerical simulations show that opening a gap may not terminate accretion of gas onto the planet (Lubow et al., 1999). Thus, a starved disk may require strong turbulence to remove this gas. But strong gas turbulence keeps the particle layer puffed-up, with $H_{p} \sim H$. For such a model the sweep-up growth times may be comparable to the drag times, increasing the likelihood that satellite embryos will be lost to the planet. Also, coagulation would be slowed down by the low particle volume density and hindered by the large relative velocities between particles of sufficient size (which would result in shattering instead of sticking). Hence, satellitesimals may have difficulty growing large enough to survive the effects of gas drag, and a significant fraction of the solid content of the disk may be lost (perhaps even before making it to the decoupling size). If and when satellites do form, they may be prevented from opening a gap (which we use in Paper II to allow for long-term survival) by the gas turbulence. As a result, even large objects would migrate due to the tidal torque of the disk. Even if objects grow large enough to open a gap in the disk, migration would continue in a time scale controlled by the viscous evolution of the strongly turbulent gas disk.

The alternative is a gas-free accretion model. However, such a model is difficult to justify. First, gas may flow through the gap. Second, turbulence may not be effective at removing the gas disk. Third, disk truncation may prevent gas from accreting onto the primary. Finally, as we discuss in this paper and in Paper II, there are numerous issues that argue in favor of the presence of gas during satellite accretion. 
There is also the issue of the source of the solids. Flow of gas through the gap may not imply infall of solids, since the dust content of the gas at late times is expected to be low (Mizuno et al., 1978; Weidenschilling, 1997). One may argue in favor of mechanisms that delay dust coagulation, but these same mechanisms may complicate planetesimal and satellitesimal formation, perhaps resulting in the loss of solids to the planet, or even prevent the planet itself from forming. Also, the high specific angular momentum gas flowing into the planet after gap-opening would orbit well outside the centrifugal radius (see Sect. 3), whereas most of the mass in the satellite system is inside this radius. Furthermore, strong turbulence would increase the size of the disk until it is cut-off by resonant or nonresonant tidal effects possibly at $\sim R_{\mathrm{H}} / 2$.

On the other hand, Mosqueira et al. (2000) considered the feeding of large planetesimals, which would not be coupled to the gas and whose dynamics have to be followed independently. However, such a model may need to be fine-tuned if it is to lead to a sufficient starved disk capture rate instead of resulting in capture by the planet or removal by gravitational scattering (as needed to produce the Oort cloud). Since planetesimals in the outer solar system scatter in a time scale of a million years (Gladman and Duncan, 1990), it must be shown that there would be enough planetesimals arriving a million years after the formation of Jupiter to make the Galilean satellites. While the delivery of material from the solar nebula to Jupiter probably lasted several million years (Wuchterl et al., 2000), the amount of material arriving late is still a small fraction of the total. Late arriving material has been proposed to explain the large core of Neptune (Lissauer et al., 1995), but the issue that concerns us here is the time scale over which the region outside Neptune was cleared of planetesimals (recent work indicates that planetesimals outside proto-Neptune's feeding zone may be prevented from colliding with it (Ida et al., 2000)).

In addition, lengthening the satellite formation time exposes the proto-satellites to prolonged bombardment from the Roche-lobe. This is especially a problem for Jupiter's satellite system, where the velocity of the incoming projectiles may be large enough to shatter objects as large as $\sim 1000 \mathrm{~km}$ (Greenberg et al., 1977). In contrast, our model has satellite embryos forming quickly, so that their exposure to potentially disruptive bombardment is limited.

The angular momentum of the satellite system also presents a serious challenge to a starved disk model, particularly one that is fed by planetesimals with small net angular momentum with respect to the protoplanet. Even if the planetesimals originate from the outer regions of the solar nebula, one might still expect preferential capture of retrograde objects. In our model, the angular momentum of Callisto and Iapetus may ultimately be the result of the tide of the Sun on gas flowing into the giant planet from the
Roche-lobe. Since the starved disk is sporadically fed by large planetesimals uncoupled to the gas, the Sun's torque may not be available to this model.

Even if all the above issues are resolved, there is no guarantee that one would end up with satellite systems like those of Jupiter and Saturn. Other issues such a model has difficulties coping with include the locations of the irregulars, the low densities of saturnian satellites other than Titan (especially that of Iapetus), the different compositions and spectra of regular and irregular satellites (see Section 9 for a discussion), the concentration of mass in Titan, the capture of Hyperion into a 4:3 mean-motion resonance with Titan, the endogenic activity of the small moons of Saturn, the mass to distance relationship in the uranian and inner saturnian satellites (see Paper II), the location of the outermost satellite of each planet, the location of the largest satellite of each planet, the relative scarcity of regular satellites far from the planet, the formation of a fully differentiated Ganymede but only a partially differentiated Callisto, and the sizes, positions, and densities of the Galilean satellites. Using such a model one may argue that the compositional gradation among the Galilean satellites is a consequence of impact (Stevenson et al., 1986). That is, impacts close to the planet from late arriving high-velocity planetesimals might preferentially re-accrete rock. However, this argument is hard to apply to Saturn, where the satellite density increases fairly systematically out to Titan and then decreases again for Iapetus. Conversely, one might use collisions with a differentiated satellite to produce icy satellites, but one would then need to explain why this did not happen for Jupiter.

To close this discussion, we point out that regardless of the details of such a model, it is likely that it would predict a partially differentiated state for Titan. Accretion onto Titan would have occurred even slower and at a lower temperature (both of the nebula and of the object) than Callisto's accretion. Furthermore, a starved disk model may preclude the presence of significant amounts of ammonia in Titan or any other satellite (Prinn and Fegley, 1981). Therefore, one is led to expect a partially differentiated (Callistolike) Titan. Recent work (Mousis et al., 2002) offers an alternative to the model of Prinn and Fegley $(1981,1989)$. These authors suggest that both $\mathrm{NH}_{3}$ and $\mathrm{CH}_{4}$ (but not $\mathrm{CO}$ ) might have been derived directly from the solar nebula. If so, ammonia would also be likely to be present in Callisto, and one would again need to explain the putative difference between Callisto and Titan in this regard. In any case, this work relies on unknown values of the nebula turbulence as well as the initial ratio of $\mathrm{N}_{2} / \mathrm{NH}_{3}$ in the protosolar nebula. Other possible differences between large satellites include the effects of planetary tides and satellite rheology. However, in each of these cases one is forced to fine-tune the model to ensure that both Ganymede and Titan would differentiate, but Callisto would end up partially differentiated. One may argue that the possibility still exists that Callisto 
will turn out to be fully differentiated. In that case, the starved disk model would lose its main motivation.

\section{Discussion and conclusions}

We have used a consistent model for the accretion of regular satellites of Jupiter, Saturn, and Uranus. We do exclude coorbitals, small regular satellites found close to the giant planet, and the satellites of Neptune from consideration. The coorbitals will require a separate treatment, which we do not attempt here. Small satellites close to the planet are likely to have undergone significant collisional or tidal evolution after their formation, so they may not provide useful constraints on satellite formation models. Finally, we have excluded the satellites of Neptune due to the likely capture of Triton from heliocentric orbit (Goldreich et al., 1989; McKinnon, 1984); nevertheless, we expect that originally Neptune's satellite system may have been largely analogous to that of Uranus. Hence, it may not be surprising that Nereid (a regular satellite disturbed by Triton's capture) is spectrally like the satellites of Uranus and unlike Centaur objects (Brown et al., 1998).

Though a variety of accretion scenarios arise out of our model, we argue that this is not tantamount to special pleading for each satellite; rather, the various possibilities all derive from the parameter space available to the model. In our view, the complexity of the model is justified by the observations. Of course, any satellite formation model must contend with several uncertain parameters, most notably the turbulence parameter $\alpha$, and ours is no exception. The aim of this study is not so much to explore the available parameter space as to describe a consistent model with a number of desirable features. Our model does rely on the validity of a two-component subnebula. However, we argue that such a model may be natural inasmuch as it can account for the location of the largest satellites and the outermost regular satellites of Jupiter, Saturn, and Uranus (see also Paper II), the anomalously large angular momentum of Callisto, its partially differentiated state, the capture of Hyperion into resonance, the large separation between Titan and Iapetus, the absence of large satellites inside of Titan, and the relative scarcity of regular satellites far from the planet. It is certainly possible to increase the size of the inner disk or of the transition region. Considering that Callisto is likely to have migrated inward, there is room for an inner disk significantly more extended than we have chosen in this paper. One must keep in mind, however, that the compositional gradient of the Galilean satellites argues in favor of a model where most of the mass in the disk is close to the planet.

We investigate a model for giant planet regular satellite formation in which the satellites accrete in the presence of a dense (optically thick given gaseous opacity) inner gaseous disk extending out to the planet's centrifugal radius and from an extended, low density (optically thin given gaseous opacity) outer disk extending out to a fraction of the planetary Hill radius. The accretion of the satellites takes place at the tail end of the formation of the giant planet, at a time of heavy Roche-lobe planetesimal bombardment; however, the bulk of the materials in the satellites is derived from condensables left behind by planetesimal break-up or dissolution in the giant planet's extended, collapsing envelope, from which the circumplanetary gas disk formed in a relatively short time. As an initial condition, we assume a minimum mass cosmic mixture model to estimate the mass of the disks of Jupiter and Saturn and consider weak subnebula evolution after that; and in light of the high- $Z$ enhancement of the giant planets, we also consider increased solid concentrations by a factor of 3-4. Our minimum mass model uses Io, Europa (reconstituted), and Ganymede to set the mass for the inner disk of Jupiter, and Titan for the inner disk of Saturn. For the outer disks of Jupiter and Saturn, we use a minimum mass disk model given by $\sim 100 M_{\text {Callisto }}$ for Jupiter and $\sim 200 M_{\text {Iapetus }}$ for Saturn, containing enough condensables to form Callisto and (icy) Iapetus, respectively. This may be appropriate because satellites forming in the outer disk may be prevented from migrating to the inner disk (see Paper II). We take the similarity of the reconstituted satellite mass ratio between Jupiter and Saturn $(\sim 3.7)$ and the ratio of the atmospheric envelopes of these two planets as an indication that (a) the same general process applied to both of their satellite systems, and (b) the amount of solid material left in the disk is related to the amount of gas in the planet's envelope. Similarly, the atmospheric envelope mass ratio between Saturn and Uranus $(\sim 18)$ compares well with the ratio of the masses in the satellite systems of these two planets $(\sim 15)$. This leads us to use a minimum mass disk model extending out to Uranus' centrifugal radius (and not to the present location of Oberon, given that the uranian satellites probably migrated inward) to estimate the gas surface density present in the uranian inner disk subnebula.

To determine the size of the outer disk, we rely on the locations of the irregular satellites of the giant planets. Connecting their location to the size of the disk allows us to explain the lack of irregulars closer to the planet. With this in mind, we expect that the outer disks of Jupiter and Saturn extended out to $\sim R_{\mathrm{H}} / 5$. It is possible that the disk extended farther out and resonant (such as an $m=2$ vertical resonance; Lubow (1981)) or nonresonant tidal mechanisms removed part of the outer disk.

These parameters (see also Sect. 3) can be used to compute the angular momentum of the subnebula. In Table 3 we present the angular momenta of the satellite systems of Jupiter, Saturn, and Uranus. A similar table appeared in Korycansky et al. (1991). It is important to note that the ratio of the total angular momentum (augmented and reconstituted) in the satellite systems divided by the spin angular momentum of the primary is fairly constant for all three giant planets. Our model explains the large angular momentum stored in Callisto (similar to that of Ganymede) by the 
Table 3

Angular momenta

\begin{tabular}{|c|c|c|c|}
\hline$M_{\text {neb }} /\left(M_{P}-M_{\text {core }}\right)^{\mathrm{a}}$ & $\begin{array}{c}\text { Jupiter } \\
\sim 0.03\end{array}$ & $\begin{array}{l}\text { Saturn } \\
\sim 0.03\end{array}$ & $\begin{array}{l}\text { Uranus } \\
\sim 0.03\end{array}$ \\
\hline Spin angular momentum, planet ${ }^{\mathrm{b}} J_{P}\left(\mathrm{~g} \mathrm{~cm}^{2} \mathrm{~S}^{-1}\right)$ & $4.4 \times 10^{45}$ & $7.4 \times 10^{44}$ & $1.3 \times 10^{43}$ \\
\hline Orbit angular momentum, satellites $J_{\text {sats }}$ & $4.5 \times 10^{43}$ & $9.3 \times 10^{42}$ & $1.4 \times 10^{41}$ \\
\hline Augmented satellite total ${ }^{\mathrm{c}} J_{\text {sats }}^{*} \times 100$ & $5.7 \times 10^{45}$ & $9.5 \times 10^{44}$ & $1.4 \times 10^{43}$ \\
\hline Augmented system total ${ }^{\mathrm{c}} J_{P}+\left(J_{\text {sats }}^{*} \times 100\right)$ & $1.0 \times 10^{46}$ & $1.7 \times 10^{45}$ & $2.7 \times 10^{43}$ \\
\hline Total nebula angular momentum $J_{\text {neb }}$ & $7.8 \times 10^{45}$ & $9.5 \times 10^{44}$ & $2.2 \times 10^{43}$ \\
\hline inner disk & $4.8 \times 10^{45}$ & $9.0 \times 10^{44}$ & $2.2 \times 10^{43}$ \\
\hline outer disk & $3.0 \times 10^{45}$ & $5.5 \times 10^{43}$ & - \\
\hline Specific angular momentum, planet $J_{P} / M_{P}\left(\mathrm{~cm}^{2} \mathrm{~s}^{-1}\right)$ & $2.3 \times 10^{15}$ & $1.3 \times 10^{15}$ & $1.5 \times 10^{15}$ \\
\hline$J_{\text {sats }}^{*} / M_{\text {sats }}^{*}$ & $1.1 \times 10^{17}$ & $6.8 \times 10^{16}$ & $1.5 \times 10^{16}$ \\
\hline$J_{\mathrm{neb}} / M_{\mathrm{neb}}$ & $1.4 \times 10^{17}$ & $6.8 \times 10^{16}$ & $2.7 \times 10^{16}$ \\
\hline$\left(J_{\text {sats }}^{*} \times 100\right) / J_{P}$ & 1.3 & 1.3 & 1.3 \\
\hline \multirow[t]{2}{*}{$J_{\text {neb }} / J_{P}$} & 1.8 & 1.3 & 1.7 \\
\hline & Ganymede & Titan & Titania \\
\hline \multirow[t]{2}{*}{$J_{\text {sat }} / J_{P}$} & $3.9 \times 10^{-3}$ & 0.012 & $4.3 \times 10^{-3}$ \\
\hline & Callisto & Iapetus & Oberon $^{\mathrm{d}}$ \\
\hline$J_{\text {sat }} / J_{P}$ & $3.8 \times 10^{-3}$ & $2.5 \times 10^{-4}$ & $4.3 \times 10^{-3}$ \\
\hline
\end{tabular}

${ }^{\text {a }}$ Jupiter, Saturn core mass $=12 M_{\oplus}$. Uranus core mass $=10 M_{\oplus}$.

${ }^{\mathrm{b}}$ Calculating using density moments from Hubbard and Marley (1989).

${ }^{\mathrm{c}}$ Reconstituted for lost volatiles and augmented by gas.

${ }^{\mathrm{d}}$ Note that Oberon is in the inner disk.

angular momentum initially present in the outer disk of Jupiter. $^{2}$

At present, the best candidate mechanism to generate turbulence and outward angular momentum transport is an entropy gradient in the subnebula leading to a nonbarotropic equation of state (Lovelace et al., 1999; Li et al., 2000; Klahr and Bodenheimer, 2001). However, it is still unclear how a global radial entropy gradient can drive turbulence; it is possible that at least close to the planet 3-D effects in the presence of both a vertical and a strong radial entropy gradient will result in the generation of turbulence and outward transport of angular momentum (though one might expect inward angular momentum transport, e.g., Quataert and Chiang, 2000). It must be kept in mind, however, that a model that transports angular momentum by the generation of large scale vortices may not be described by the $\alpha$ model prescription (Klahr and Bodenheimer, 2001). One particular issue with this mechanism is that the dissipation of vortices might tend to surpress the vertical entropy gradient. In any case, such a model may lead to turbulence that is a function of position and time. Following giant planet accretion, the subnebula turbulence is likely to subside (perhaps to $\alpha$ values of $10^{-6}-10^{-4}$ ), thus allowing the subnebula to cool down and particle coagulation to take place, possibly in a regime of efficient particle sticking.

To the extent that a hot subnebula with strong turbulence hinders the growth of particles (due to the large relative particle velocities and the depletion of frost-covered silicate

\footnotetext{
${ }^{2}$ Since there are no outer uranian satellites, we did not estimate the angular momentum of the outer uranian disk. Nonetheless, it is possible that Oberon received some condensables from the outer disk.
}

particles), the subnebula's condensable mass fraction may not have changed significantly as it cooled, as long as most of the mass resided in the form of small particles strongly coupled to the gas. This statement is not necessarily inconsistent with a concurrent drop in opacity, since sufficiently small particles may still coagulate, whereas particle growth may have trouble getting past a given size (Weidenschilling and Cuzzi, 1993; Suttner and Yorke, 2001). It must be stressed, however, that the problem of the dust opacity and coagulation of particles in the subnebula remains to be studied. After cooling, isothermal portions of the disk (outside Callisto and Titan) should be nearly quiescent, with low gas viscosity and long orbital decay times. In this respect, we interpret the cut-off in the distribution of irregular satellites of Jupiter and Saturn as suggestive of a fairly sharp decrease in the outer disk gas density in the neighborhood of $R_{\mathrm{H}} / 5$. Such a density gradient is consistent with laminar gas flow at that location. On the other end, because the inner disk of the giant planets (except perhaps in the case of Uranus) is nonisothermal both radially and vertically, weak turbulence is expected there at least as long as the gas remains optically thick (with $\Sigma>10^{4} \mathrm{~g} \mathrm{~cm}^{-2}$ ). Such a turbulence model may lead to a fairly flat surface density profile. ${ }^{3}$

Given our subnebula parameters, we show that gas drag dominates migration for particles $<500-1000 \mathrm{~km}$, and the tidal torque of the gas disk is stronger for larger objects. In the inner disk, dust and rubble are swept up fast enough to

\footnotetext{
${ }^{3}$ One may also consider a turbulence-free model in which satellite tidal torques would provide the viscosity (Goodman and Rafikov, 2001). In Paper II, we advance a related mechanism to clear the gas disk.
} 
form a $1000-\mathrm{km}$ satellite embryo in $<10^{3}$ years for Jupiter and $<10^{4}$ years for Saturn, as long as some settling of the particle layer takes place. This time scale may be sufficiently short for the embryo to survive the effects of gas drag and planetesimal bombardment from the Roche-lobe. Once the embryo reaches $\sim 1000 \mathrm{~km}$ in size it will capture a significant fraction of the satellitesimals that migrate inward into its feeding zone due to gas drag, making it likely that a considerable fraction of the condensable mass in the disk ends up in the satellite system instead of being lost to the planet. Thus, roughly speaking, a minimum mass model may in fact apply to the formation of satellites.

We expect that satellites stopped growing when either the disk ran out of accretable materials or an outer embryo choked off the growth of proto-satellites inside of its orbit. The latter possibility (perhaps in combination with collisional disruption close to the planet) may explain the sequence of sizes of the Galilean satellites and the observation that the largest satellites of both Saturn and Jupiter occur just inside the centrifugal radius: Outside this radius the gas density decrease may lead to smaller satellites, while inside this radius the growth of a satellite may be limited by its outer neighbor (e.g., Ganymede may have prevented Europa from growing to Io size). Although it is possible that for the largest satellites partial vaporization of infalling satellitesimals and subnebula gap-opening may have reduced the mass accretion rate, it is unlikely that these processes were able to terminate accretion altogether. Nevertheless, for the largest satellites (Ganymede and Titan) gap-opening may have lowered the accretion efficiency of inwardly drifting satellitesimals (a greater proportion of such objects may "horseshoe" past a large satellite surrounded by a gap, though this remains to be shown). Hence, the similarity in their masses may be connected with this process. If so, their final masses may not be determined solely by the total amount of mass present outside their orbit and inside the orbit of its outer neighbor. Perhaps both factors come into play.

Our model forms Ganymede in Jupiter's inner disk in $10^{3}-10^{4}$ years at a temperature of $\sim 250 \mathrm{~K}$ and Titan in Saturn's inner disk in $10^{4}-10^{5}$ years at a temperature of $\sim 100 \mathrm{~K}$. The upper bound is computed by estimating the size of the region from which a satellite draws materials and then computing the time scale for embryos to drag that distance and form the satellite. The lower bound is obtained by assuming that once an embryo grows to a size such that it can capture most of the satellitesimals that drift into its feeding zone, its growth accelerates. Also, the upper end of the range may apply inasmuch as we may have significantly overestimated the surface gas density for the inner disk. In the case of Titan and Ganymede, we suggest that this accretion took place fast enough that these satellites avoided extended inward migration (see Paper II). Thus, the satellite embryo which led to their formation may have originated from the neighborhood of the centrifugal radius of each of the two planets. If so, it may be appropriate to set the disk temperature to $250 \mathrm{~K}$ at the present location of Ganymede and to $100 \mathrm{~K}$ at the present location of Titan, as we have done in this study (Jupiter's and Saturn's cooling times are longer than the formation times for Ganymede and Titan, respectively). In that case, our model would lead us to expect that Ganymede captured most ice-rich satellitesimals that drifted into its feeding zone from cool regions of the disk. Furthermore, since the satellite tidal torques would have cleared the gas between Io and Ganymede in a timescale much shorter than the planetary cooling time, Io and Europa would have ended up nearly ice-free as observed.

In the outer disk, the low, constant temperature of the gas $(\sim 130 \mathrm{~K}$ for Jupiter and $\sim 90 \mathrm{~K}$ for Saturn) leads us to expect that it is characterized by weak turbulence driven only by the vertically thin particle layer shear (Cuzzi et al., 1993). Therefore, despite the longer dynamical times and low solid surface densities, the time scale for the formation of embryos by dust and rubble sweep-up and by drift augmented accretion of satellitesimals is about 50 times faster than the orbital decay time scales for such embryos. Consequently, we expect embryos to form quickly until the disk becomes depleted of dust and rubble. Continued growth of similarly sized embryos takes place at the Safronov time scale and leads to sizes of several hundred kilometers in a time scale comparable to their drift times. Once these embryos reach the position of proto-Callisto most of them will be accreted by it. We calculate characteristic embryo sizes by comparing the drift times of satellitesimals across twice the embryo's feeding zone to the synodic time of two objects separated by half that distance. In our model Callisto's accretion time scale is set by the inward drift of embryos 300-500 km (with the smaller embryo size the result of the longer satellitesimal drift time of a model with four times greater concentration of solids) from as far away as $\sim 150 R_{\mathrm{J}}$ (compared with $\sim 23 R_{\mathrm{J}}$ for Ganymede). Similarly, the timescale for the accretion of Iapetus is set by the drift of embryos from as far as $\sim 200 R_{\mathrm{S}}$. We show that the Safronov time scale to build embryos $\$ 500 \mathrm{~km}$ at distances $\sim 100 R_{\mathrm{J}}$ is about $10^{6}$ years. Also, the orbital decay time of such an object from that distance is $\sim 10^{6}$ years (though the time scale depends on the concentration of solids in the disk). Although for the outer disk our model leads to long formation times for full-sized satellites, the embryos that led to the satellite formed quickly, and the long formation time scale is derived from the slow accretion of dispersed embryos.

A formation time scale of $\sim 10^{6}$ years for Callisto (and $10^{6}-10^{7}$ years for Iapetus) may be slow enough to lead to a partially differentiated state for Callisto (Iapetus is mostly made of ice) consistent with the two-layer model advanced by Anderson et al. (2001), which has a clean ice layer of $\sim 300 \mathrm{~km}$ overlying a mixed ice and rock-metal interior. It must be stressed that although this model leads to large embryos hitting the proto-satellite, they do so at the escape velocity of the proto-satellite (as supposed to the hypervelocity impacts that would result from Roche-Iobe objects). 
In this energy regime, crater diameters are $\sim 3 r_{s}$, so that the penetration depth of such objects is likely on the order of their radius, with excavation depths being even smaller (Melosh, 1989). Thus, a single collision may not lead to runaway differentiation of the proto-satellite. Although impacts with satellite embryos hundreds of kilometers in size will bury heat, one expects that the top $\sim 200-\mathrm{km}$ layer of slush would redistribute the energy of accretion down to the ice I/III transition in any case. Perhaps most important, the surface of the satellite may be prevented from freezing and trapping heat inside by the presence of a thick atmosphere. If so, an accretion time of $\sim 10^{6}$ years may be long enough for the atmosphere to radiate away the energy of accretion, while avoiding a runaway greenhouse that might also lead to a fully differentiated state (Kuramoto and Matsui, 1994). It is also possible that particulate matter coupled to high specific angular momentum gas flowing through the gap after giant planet gap-opening, planetesimal capture, or ablation by the extended, long-lived gas disk lengthens the time scale of formation of regular satellites (but one should keep in mind that at late times most of the solids are likely to be in the form of planetesimals (Weidenschilling, 1997) and that Iapetus' density may be too low to be formed by material accreted directly from heliocentric orbit). Still, more work needs to be done to check whether our model can realistically lead to a partially differentiated Callisto.

Callisto could then be said to be the result of slowly assembling hundreds to thousands of volatile rich, "cold" embryos. This may account for the preferential retention of ices more volatile than water in Callisto compared to Ganymede. This also means that (possibly unlike Ganymede and Titan) both Callisto and Iapetus may have migrated large distances (see Paper II for an explanation of their locations). Lewis (1974) hypothesized that lower formation temperatures for Callisto than for Ganymede would likely have led to the accretion of solid ammonia hydrate in Callisto. Our model indeed has Callisto forming cold; however, it does so in a region of the disk disconnected from the inner, optically thick region where the temperature and density might have led to the production of reduced ices such as methane and ammonia (Prinn and Fegley, 1981). Therefore, we expect Callisto bulk composition to adhere closely to solar mixtures (though some chemistry could have taken place in the giant planet envelope), which may contain mostly oxidized ices. However, methane is known to be present in many solar system objects, and the presence of ammonia has not been ruled out (Mousis et al., 2002). It is also worth pointing out that whereas Callisto and Iapetus are likely to have accreted homogeneously (their accretion time is longer than the disk cooling time at its location), the same may not be true for Ganymede or Titan. It must be stressed that despite the different predictions that our model makes for satellites forming in the inner and outer disks, the basic processes that led to their formation are essentially the same.

Given our model, Titan's methane atmosphere may still be the result of the condensation of methane clathrate hydrate (Lewis, 1974). Another interesting if somewhat speculative possibility of our model is that Titan formed its atmosphere as a result of a collision with a volatile-rich, Iapetus-sized object. Given its location, it is unlikely that Iapetus collected all the material in Saturn's outer disk. Therefore, large embryos could have formed outside the centrifugal radius which ultimately ended up colliding with Titan. Iapetus itself may owe its dark, reddish material to the condensation of methane in the subnebula (Squyres and Sagan, 1983). Also, since our model has an optically thick inner disk, one might expect some production and outward transport of ammonia, making it it possible that ammonia is responsible for ancient volcanic plains in Tethys and Dione. However, the outer regions of the disk might not be affected. The lack of any resurfacing in Callisto and its presence in the much smaller Dione seems to indicate that Callisto lacks ammonia but Dione has it. But it must be pointed out that Rhea appears inert. It might be that Rhea received less ammonia than Dione by virtue of its location. On the other hand, it is also possible that ammonia fails to resurface larger satellites (see McKinnon, 1999, and references therein).

There is also the issue of the $\mathrm{D} / \mathrm{H}$ ratio for the satellites. While more work has to be done in this respect, to the extent that neither Callisto nor Iapetus were coupled to a thick subnebula one might expect an enhanced $\mathrm{D} / \mathrm{H}$ ratio for these satellites with respect to Ganymede and Titan. However, given the location of Iapetus, Titan probably received a substantial amount of material from the outer disk in the late stages of its accretion. Hence, it may not be surprising that the measured $\mathrm{CH}_{3} \mathrm{D} / \mathrm{CH}_{4}$ ratio in Titan's atmosphere (Coustenis, 1989) is comparable but lower than that of comets (Bockelee-Morvan et al., 1998), though later measurements point to even lower D/H ratios for Titan's atmosphere (Orton, 1992). Unlike the scenario envisioned by Prinn and Fegley (1981), which relies on the conditions prevalent in the subnebula disk to process the volatiles derived from the nebula, recent work by Mousis et al. (2002) hypothesizes that Titan derived its volatile inventory from the solar nebula itself. Because our model has Titan forming at the outer edge of the weakly turbulent inner disk, it may be compatible with either of these views. It is also possible that the present $\mathrm{D} / \mathrm{H}$ ratio in Titan's atmosphere is the result of photochemical enrichment of deuterium due to preferential retention of that isotope during methane photolysis (Lunine and Tittemore, 1993); however, this conclusion depends on the unknown degree of mixing of processed gases in the inner disk at the time of satellite formation and on the assumption that the methane in the atmosphere of Titan is not replenished from a ground reservoir as it is photodissociated (Mousis et al., 2001). Nevertheless, such a scenario remains viable within the context of our model. Furthermore, the possibility has not been ruled out that the required chemistry took place in the giant planet envelope during planetary accretion, before the formation of the accretion 
disk took place. It is also unclear whether Ganymede's D/H ratio can be expected to be substantially lower than that of Callisto, but the issue merits further study. Our model does lead one to expect about an order of magnitude greater value for the $\mathrm{D} / \mathrm{H}$ ratio of Iapetus than the protosolar value.

Because Saturn's disk is characterized by longer dynamical times and has less gas spread out over a larger distance than Jupiter's disk, our model leads to significantly longer accretion time scales for the satellites of Saturn. It is possible that no large satellites formed inside Titan because the embryo formation time scale $\sim 10^{4}$ years made them vulnerable to hypervelocity impacts close to the planet and the collisional debris was then swept by gas drag (but see Paper II for alternative explanations based on satellite migration). This mechanism may work because characteristic satellite embryos in Saturn's disk are small $(<1000 \mathrm{~km})$.

We have seen that our model has Ganymede and Titan forming in the inner disk and Callisto and Iapetus in the outer disk. It also has Hyperion forming in the transition region. This is significant because it fits well with the formation model of Lee and Peale (2000), which has Hyperion captured into resonance by proto-Titan in the presence of a strong gas density gradient. Given our subnebula parameters, we show that Titan satisfies the criterion for gas drag resonance capture of satellitesimals as small as a kilometer, whereas Ganymede requires that the object be significantly larger. This may explain the presence of Hyperion at Titan's 4:3 mean-motion resonance location. Ganymede itself may have captured such an object into resonance, which was subsequently lost because its orbit was unstable or due to collisional scattering. On the other hand, Callisto may have formed in a region of the disk where the gas drag was weak enough that typical satellitesimal eccentricities were larger than the critical value such that resonance capture probability becomes small. If so, this may place constraints on the environment that gave rise to the regular satellites of giant planets. This issue merits further work.

We extend this model to the satellites of Uranus. Though for Uranus the mass for the inner or outer disks may not have been enough to form a Titan or an Iapetus, we find significant similarities between the uranian satellite system and the inner saturnian satellites. We defer further discussion of this issue to Paper II. Here we simply point out that we interpret the gap between the centrifugal radius and the outermost uranian regular satellite as suggestive of substantial inward satellite migration. In the inner disk, satellite formation times may have been sufficiently fast $\left(\sim 10^{5}\right.$ years) to explain why Titania (Uranus' largest satellite) shows evidence of resurfacing.

The question of the silicate fraction of the satellites is likely to be quite complicated with many thorny issues that are beyond the scope of this paper (see McKinnon et al., 1997, for a review). Here we simply point out that our model seems to fit better with the view that most of the regular satellites of giant planets are not water-deprived but water-enriched. By this we mean that regular satellites forming in regions of the subnebula where water-ice condensation had taken place probably had more water available to them than given by solar mixtures, even if they lost some of that water in the process of accretion. As has been done before (e.g., Podolak et al., 1993), we note that all the regular satellites of giant planets with the exception of Io and Europa have silicate fractions well below those of Triton, Pluto, and Charon (though at least Triton may have lost a significant amount of water during its history). There are several mechanisms that can lead to water enrichment, and we suggest that their interplay may have the best chance of explaining the observed silicate fractions of the regular satellites of the giant planets. First, either ice ablation of planetesimals hitting the envelope of the giant planet, dust settling, preferential break-up of icy objects, or ablation of planetesimals passing through the gas disk can add water content relative to silicates to satellites forming far from the planet (this may have played a major role in the case of Iapetus, which might explain its low density, and to a lesser extent in the case of Callisto). Second, more water may be available under nebular conditions applicable to satellites forming in the inner disk than given by solar mixtures (this may apply to Ganymede, Titan, and to a lesser degree the uranian satellites). Third, inhomogeneous accretion may lead to selective loss of silicates (this may be applicable to the inner saturnian satellites and perhaps to Miranda, though it is more likely that collisional break-up followed by reaccretion left Miranda underdense). It is also possible that the circumplanetary disks of the giant planets as a whole preferentially lost silicates due to inhomogeneous accretion, leaving the satellites relatively water-rich. Finally, the disk might have been water-enriched because kilometer-sized rocky objects penetrated too deep into the giant planet envelope to be deposited in the accretion disk. However, much more work will have to be done to check into these issues.

We have already stated the possibility that Callisto and Iapetus derived some condensables from material captured from heliocentric orbit. However, this may be unlikely. ${ }^{4}$ Instead, we expect that this mass source was a small contribution to the bulk of the satellites. Nonetheless, this may not mean that captured objects or debris originating from them did not leave their mark on Callisto and Iapetus (and the outer satellites of Uranus). It is possible that the leading and trailing asymmetries present in the outer uranian satellites (Buratti and Mosher, 1991), perhaps Callisto (Bell, 1984), and Iapetus (Buratti et al., 2002, and references therein) may be the result of interactions with material originating from retrograde satellites exterior to their orbits (Cruikshank et al., 1983). Buratti et al. (2002) argue in favor of this alternative to explain the extreme leading and trailing composition asymmetry of Iapetus. If so, this may explain

\footnotetext{
${ }^{4}$ Though we are currently investigating the possibility that the ablation of planetesimals passing through the disk contributed significantly to the mass of solids in the satellite system.
} 
why the spectra of Iapetus and Hyperion (Hyperion tumbles chaotically (Wisdom et al., 1984), but it may also have undergone interactions with such a source of material) fit with a two-component model of an icy satellite and D-type material (and does not fit with Phoebe's visual spectrum, which is flat and more similar to a C-type asteroid). The recent discovery of collisionally evolved retrograde saturnian (Gladman et al., 2001) and uranian (Gladman et al., 2000) satellites may provide such a source. Thus, a scenario which has regular satellites forming in a gaseous accretion disk around the planet and in which the irregulars are captured objects may account for the spectra and other observed properties of both regular and irregular satellites, a division which may pose a serious challenge to a starved disk model (particularly a gas free one).

We briefly consider the alternative model that Callisto's accretion time scale was set by the time scale over which solids were fed into the system and that all the regular satellites of Jupiter and Saturn formed from a starved disk. We conclude that this scenario faces significant hurdles. Nevertheless, the issue needs to be settled. We propose that a test of this alternative model is whether or not Titan is differentiated. Our model leads us to the conclusion that, although Titan took considerably longer to form than Ganymede (due to the lower surface density and longer dynamical times of Saturn's disk compared to that of Jupiter), it still formed in a fairly short time scale $\left(<10^{5}\right.$ years), making it very likely that it is fully differentiated. On the other hand, the starved disk model implies a long accretion time for Titan with low temperatures and small quantities of $\mathrm{NH}_{3}$ present. While it may be possible to avoid this conclusion by fine-tuning parameters such as the size of the impactors, the amount of ammonia present, or other satellite properties, we suggest that such a model is likely to predict a partially differentiated (Callisto-like) Titan. The Cassini mission may resolve this issue conclusively.

\section{Acknowledgments}

We thank Jeffrey Cuzzi, Kevin Zahnle, Doug Lin, Peter Bodenheimer, Sandy Davis, Dale Cruikshank, and Jeff Moore for discussions. One of us (I.M.) had numerous, very helpful discussions with Dave Stevenson. We also thank Jeffrey Cuzzi, Kevin Zahnle, and Jack Lissauer for reading the manuscript and suggesting improvements, and Steve Squyres for discussions, comments on the manuscript, and generous support of this work. This research was supported by a grant from the Planetary Geology and Geophysics program.

\section{Appendix A: velocity dispersion in the outer disk}

Because of the effects of gas drag, we expect the outer disk will be populated by similar-sized embryos; smaller objects would be quickly incorporated into larger ones by drift augmented accretion. Given this, we ask what the velocity dispersion of such objects is. The mean eccentricities $e$ and inclinations $i$ are determined by the balance between excitation due to gravitational encounters and damping due to gas drag and inelastic collisions. Using the average time rate of change of $e$ and $i$ determined by Adachi et al. (1976, their Eq. (4.15)), as well as their mean-square variations (Hayashi et al., 1977, Eq. (5.24)), we have

$$
\begin{aligned}
\frac{d e^{2}}{d t}= & \frac{0.47}{\tau_{c g}} \frac{1}{i \sqrt{i^{2}+e^{2}}} \\
& -\frac{2 e^{2}}{\tau_{g}}(0.77 e+0.64 i+\eta)-\frac{e^{2}}{\tau_{c}}=0, \\
\frac{d i^{2}}{d t}= & \frac{0.094}{\tau_{c g}} \frac{1}{i \sqrt{i^{2}+e^{2}}} \\
& -\frac{i^{2}}{\tau_{g}}(0.77 e+0.85 i+\eta)-\frac{i^{2}}{\tau_{c}}-0,
\end{aligned}
$$

where the characteristic time scales due to gas $\tau_{g}$, inelastic collisions $\tau_{c}$, and gravitational interactions $\tau_{c g}$ are given by

$$
\begin{aligned}
\tau_{g} & =\frac{16 \rho_{s} r_{p} c a^{2}}{3 C_{D} G M_{P} \Sigma}, \\
\tau_{c} & =\frac{1}{\sqrt{2} n \sigma u}, \\
\tau_{c g} & =\frac{3 M_{P}^{2}}{36.64 \pi^{2} a^{2} \Omega \sum_{s} \rho_{s} r_{p}^{3} \ln \Lambda_{r}},
\end{aligned}
$$

where $n=3 \Sigma_{s} \Omega /\left(8 \pi \rho_{s} r_{p}^{3} u\right)$ is the number density of particles, $\sigma=4 \pi r_{p}^{2}(1+\Theta)$ is the cross-section with $\Theta=$ $v_{\text {esc }}^{2} / 2 u^{2}$ the Safranov parameter, $u^{2}=\left\langle e^{2}+i^{2}\right\rangle v_{K}^{2} / 2$ is the mean-square velocity, and

$$
\Lambda_{r}=\frac{\left\langle e^{2}+i^{2}\right\rangle M_{P}}{4 a m_{s}} s=\frac{\left\langle e^{2}+i^{2}\right\rangle M_{P} u}{8 \pi a \Omega \Sigma_{s} r_{p}^{2}},
$$

with the mean free path $s=\sqrt{2} \rho_{s} r_{p} u / 3 \Omega \Sigma_{s}$. We find solutions for the cases in which gravitational interactions are balanced by (a) inelastic collisions, (b) gas drag, and (c) both inelastic collisions and gas drag. For these solutions we assume that $\eta \ll e, i$, but we note that this may be only marginally true. For case (a) in which we balance gravitational interactions with inelastic collisions only, we have (Hayashi et al., 1977)

$$
e=\sqrt{5} i=0.99\left(\tau_{c} / \tau_{c g}\right)^{1 / 4} .
$$

Here for a 500-km object at $a=150 R_{\mathrm{J}}$, we find that $e \sim$ 0.23 and $i \sim 0.1$. For case (b) we get (Hayashi et al., 1977)

$$
e=1.7 i=0.78\left(\tau_{g} / \tau_{c g}\right)^{1 / 5} .
$$

We find that for a 500-km satellitesimal at $a=150 R_{\mathrm{J}}$ with a density of $\rho_{s}=1.5 \mathrm{~g} \mathrm{~cm}^{-3}, e \sim 0.17$ and $i \sim 0.1$. For case 


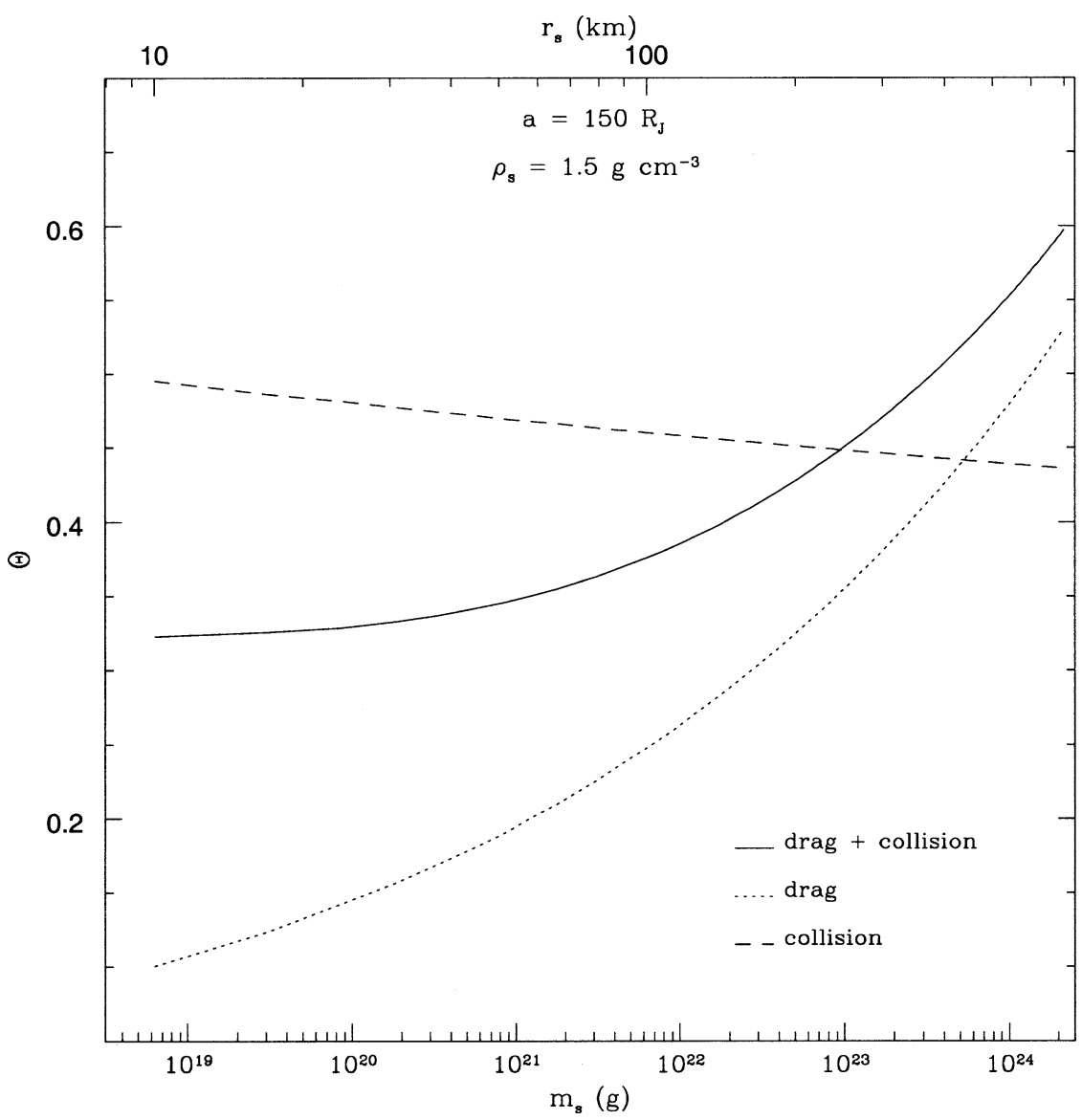

Fig. 7. Safronov parameter versus satellitesimal mass and radius (assuming $\rho_{\mathrm{s}}=1.5 \mathrm{~g} \mathrm{~cm}^{-3}$ ) in the outer disk. Gravitational encounters are balanced by inelastic collisions (dashed curve), gas drag (dotted curve), and both gas drag and inelastic collisions (solid curve)

(c) we cannot decouple the characteristic times from Eqs. (A1) and (A2) in order to find a simple relationship between $e$ and $i$. We find a good estimate in the satellitesimal size range we are interested in to be

$$
e \sim 1.9 i \sim 0.83\left(\tau_{g} / \tau_{c g}\right)^{1 / 5}\left(1.1+\tau_{g} / 2 e \tau_{c}\right)^{-1 / 5} .
$$

In this case for a $500-\mathrm{km}$ object at $a=150 R_{\mathrm{J}}$, we find that $e \sim 0.16$ and $i \sim 0.084$.

In all three cases, we iterate to find solutions for $e$ and $i$. We have solved these three cases for a range of satellitesimal masses at $a=150 R_{\mathrm{J}}$ and plotted them versus the Safranov parameter in Fig. 7. The satellitesimals are assumed to have a density of $\rho_{s}=1.5 \mathrm{~g} \mathrm{~cm}^{-3}$. The dashed line represents the solution to case (a). The Safranov parameter decreases with satellitesimal mass. This is due to the fact that there are fewer inelastic collisions for larger particles. The dotted line corresponds to case (b), where we balance the pumping of $e$ and $i$ due to gravitational interactions and gas drag. Here $\Theta$ increases with satellitesimal mass. Likewise case (c), which combines both effects, corresponds to the solid line.

For all cases we find $\Theta<1$, indicating that over this range of satellitesimal sizes the focusing is weak. Interest- ingly, the ratio of drag time $\tau_{\mathrm{gas}}$ to collision time $\tau_{c}$ can be written as

$$
\frac{\tau_{\mathrm{gas}}}{\tau_{c}} \sim 6\left(\frac{e}{i}\right)\left(\frac{130 \mathrm{~K}}{T}\right)^{3 / 2}\left(\frac{150 R_{\mathrm{J}}}{a}\right)^{3 / 2} .
$$

Notice that this is independent of particle size. Also note that since $e / i \approx$ constant the above ratio is independent of the velocity dispersion. Likewise, the Safronov accretion time scale is independent of velocity dispersion for $F_{g} \sim 1$.

\section{Appendix B: collisional breakup of satellitesimals}

Sufficiently large impacts, even occurring at velocities close to the escape velocity of the target, have the potential for fragmentation of the original body. The outcomes of such impacts are most often characterized by a dimensionless parameter

$$
f_{1}=A\left(\frac{E_{\mathrm{disr}}}{E_{p}}\right)^{b}
$$

where $E_{\text {disr }}$ is the disruption energy of the target, $E_{p}$ is the impact energy imparted by the projectile, $A$ is a constant of 
order unity, and $b \sim 0.8-1.25$ for various target materials (e.g., Fujiwara et al., 1977; Melosh, 1989; Davis et al., 1999). The parameter $f_{1}$ is the ratio between the mass of the largest collisional fragment and the mass of the original body. For $f_{1} \simeq 1$, the collision leads to erosion of the target; for $f_{1} \simeq 0.5$ the target has suffered a significant break-up (threshold of catastrophic disruption); and $f_{1} \ll 1$ indicates a catastrophic break-up of the target into small fragments (Melosh, 1989). The disruption energy of the target is generally taken to be a function of the target's strength $Y$ only; however, for large objects one must consider the self-gravity effects of the target, which we may express for spherical objects with uniform mass as

$$
E_{\mathrm{disr}} \sim \frac{4}{3} \pi r_{s}^{3}\left[c_{1} Y+\frac{4}{3} c_{2} \pi G \rho_{s}^{2} r_{s}^{2}\right],
$$

where $c_{1}$ and $c_{2}$ are dimensionless constants of order unity. The crossover size such that the strength of the target is on the same order as its gravitational binding energy has been found to range from radii of several hundred kilometers to as small as a few hundred meters (Ahrens and Love, 1996). Assuming $c_{1}=c_{2}=3 / 5$ (gravitational binding) in Eq. (B2), one finds that the transition size may be expressed as $r_{s} \sim$ $\left(3 Y / 4 \pi G \rho_{s}^{2}\right)^{1 / 2}$, which yields objects tens of kilometers in size for strengths in the MPa range.

As an example, we consider the collision of two equally sized objects $\left(r_{s}>100 \mathrm{~km}\right)$ in the outer disk assuming that target strength is unimportant. The energy imparted into the target is taken to be half of the total collisional kinetic energy $E_{p}=m_{p} v_{p}^{2}(1+2 \Theta) / 4 \approx m_{p} v_{\mathrm{esc}}^{2} / 2$, where we assume the remaining half of the energy remains in the projectile and where the Safronov parameter is taken to be $\Theta=0.5$ as a representative value (see Fig. 7). We find that $f_{1} \simeq 0.5$ 0.7 , which satisfies the criterion for partial disruption of the target. This suggests that collisional disruption may have played an important role in determining the accretion time scale for satellite embryos in the outer disk.

\section{References}

Adachi, I., Hayashi, C., Nakazawa, K., 1976. The gas drag effect on the elliptic motion of a solid body in the primordial solar nebula. Prog. Theor. Phys. 56, 1756-1771.

Ahrens, T.J., Love, S.G., 1996. Strength versus gravity dominance in catastrophic impacts. Lunar. Planet Sci. 27, 1-2.

Anderson, J.D., Schubert, G., Jacobsen, R.A., Lau, E.L., Moore, W.B. Sjogren, W.L., 1998. Distribution of rock, metals, and ices in Callisto. Science 280, 1573-1576.

Anderson, J.D., Jacobson, R.A., McElrath, T.P., Moore, W.B., Schubert, G., Thomas, P.C., 2001. Shape, mean radius, gravity field, and interior structure of Callisto. Icarus 153, 157-161.

Artymowicz, P., Lubow, S.H., 1996. Mass flow through gaps in circumbinary disks. Astrophys. J. 467, L77.

Balbus, S.A., Hawley, J.F., 1991. A powerful local shear instability in weakly magnetized disks. II. Nonlinear evolution. Astrophys. J. 376, 223.

Balbus, S.A., Hawley, J.F., Stone, J.M., 1996. Nonlinear stability, hydrodynamical turbulence, and transport in disks. Astrophys. J. 467, 76.
Beatty, J.K., ed., 1999. The New Solar System. Cambridge Univ. Press, Cambridge.

Bell, S.F., 1984. Callisto: Jupiter's Iapetus? Lunar and Planet Sci. 15, 44-45.

Bockelee-Morvan, D., Gautier, D., Lis, D.C., Young, K., Keene, J., Phillips, T.G., Owen, T., Crovisier, J., Goldsmith, P.F., Bergin, E.A., Despois, D., Wooten, A., 1998. Deuterated water in Comet C/1996 B2 (Hyakutake) and its implications for the origin of comets. Icarus 133, 147-162.

Bodenheimer, P., Pollack, J.B., 1986. Calculations of the accretion and evolution of the giant planets: the effects of solid cores. Icarus 67, 391-408.

Brown, M.E., Koresko, C.D., Blake, G.A., 1998. Detection of water ice on Nereid. Astrophys. J. 508, L175-L176.

Bryden, G., Chen, X., Lin, D.N.C., Nelson, R.P., Papaloizou, C.B., 1999. Tidally induced gap formation in protostellar disks: gap clearing and suppression of protoplanetary growth. Astrophys. J. 514, 344-367.

Bryden, G., Rozyczka, M., Lin, D.N.C., Bodenheimer, P., 2000. On the interaction between protoplanets and protostellar disks. Astrophys. J. 540, 1091-1101.

Buratti, B.J., Mosher, J.A., 1991. Comparative color and albedo maps of the uranian satellites. Icarus 90, 1-13.

Buratti, B.J., Hicks, M.D., Tryka, K.A., Sittig, M.S., Newburn, R.L., 2002. High-resolution 0.33-0.92 $\mu \mathrm{m}$ spectra of Iapetus, Hyperion, Phoebe, Rhea, Dione, and D-type asteroids: how are they related? Icarus 155, 375-381.

Cabot, W., 1996. Numerical simulations of circumstellar disk convection. Astrophys. J. 465, 874-886.

Cameron, A.G.W., 1978a. Physics of the primitive solar accretion disk. Moon and Planets 18, 5-40.

Cameron, A.G.W., 1978b. Physics of the primitive solar nebula and of giant gaseous protoplanets, in: Gehrels, T. (Ed.), Protostars and Planets, Univ. of Arizona Press, Tucson, pp. 453-487.

Cameron, A.G.W., Pine, M.R., 1973. Numerical models of the primitive solar nebula. Icarus $18,377-406$.

Canup, R.M., Ward, W.R., 2000. A possible impact origin of the uranian satellite system. Proceedings of the 32nd DPS meeting, Pasadena, California.

Carlson, R.W., 1999. A tenuous carbon dioxide atmosphere on Jupiter's moon Callisto. Science 283, 820-821.

Cassen, P., Moosman, A., 1981. On the formation of protostellar disks. Icarus 48, 353-376

Cassen, P., Pettibone, D., 1976. Steady accretion of a rotating fluid. Astrophys. J. 208, 500-511.

Cassen, P., Summers, A., 1983. Models of the formation of the solar nebula. Icarus 53, 26-40.

Coradini, A., Federico, C., Luciano, P.1982. Ganymede and Callisto: Accumula-tion heat content, in: Coradini, A., Fulchignoni, M. (Eds.), The Comparative Study of Planets, D. Reidel, Dordrecht.

Coradini, A., Cerroni, P., Magni, G., Federico, C., 1989. Formation of the satellites of the outer Solar System-sources of their atmospheres, in: Atreya, S.K., Pollack, J.B., Matthews, M.S. (Eds.), Origin and Evolution of Planetary and Satellite Atmospheres, Univ. of Arizona Press, Tucson, pp. 723-762.

Coustenis, A., Bezard, B., Gautier, D., 1989. Titan's atmosphere from Voyager infrared observations. II. The $\mathrm{CH}_{3} \mathrm{D}$ abundance and $\mathrm{D} / \mathrm{H}$ ratio from the $900-1200 \mathrm{~cm}^{-1}$ spectral region. Icarus $82,67-180$.

Cruikshank, D.P., Bell, J.F., Gaffey, M.J., Brown, R.H., Howell, R., Beerman, C., Rognstad, M., 1983. The dark side of Iapetus. Icarus 53, 90-104.

Cuk, M., Burns, J.A., 2002. Gas-assisted capture of the irregular satellites of Jupiter. Submitted to Icarus.

Cuzzi, J.N., Dobrovolskis, A.R., Champney, J.M., 1993. Particle-gas dynamics in the midplane of a protoplanetary nebula. Icarus 106, 102134.

Davis, D.R., Farinella, P., Marzari, F., 1999. The missing Psyche family: collisionally eroded or never formed? Icarus 137, 140-151. 
Dermott, S.F., Thomas, P.C., 1994. The determination of the mass and mean density of Enceladus from its observed shape. Icarus 109, 241257.

Dubrulle, B, 1993. Differential rotation as a source of angular momentum transport in the solar nebula. Icarus 106, 59-76.

Friedson, A.J., Stevenson, D.J., 1983. Viscosity of rock-ice mixtures and applications to the evolution of icy satellites. Icarus 56, 1-14.

Fujiwara, A., Kamimoto, G., Tsukamoto, A., 1977. Destruction of basalt bodies by high-velocity impact. Icarus $31,277-288$.

Gammie, C.F., 1996. Linear theory of magnetized, viscous, self-gravitating gas disks. Astrophys. J. 463, 725

Gladman, B., Duncan, M., 1990. On the fates of minor bodies in the outer Solar System. Astron. J. 100, 1680-1693.

Gladman, B., Kavelaars, J.J., Holman, M., Petit, J.-M., Scholl, H., Nicholson, P.D., Burns, J.A., 2000. The discovery of Uranus XIX, XX, and XXI. Icarus 147, 320-324.

Gladman, B., Kavelaars, J.J., Holman, M., Nicholson, P.D., Burns, J.A., Hergenrother, C.W., Petit, J.-M., Marsden, B.G., Jacobson, R., Gray, W., Grav, T., 2001. Orbital clustering for twelve newly-discovered saturnian satellites: clues to their origin. Nature 412, 163-166.

Goldreich, P., Tremaine, S., 1978. The formation of the Cassini division of Saturn's rings. Icarus 34, 240-253.

Goldreich, P., Tremaine, S., 1979. The excitation of density waves at the Lindblad and co-rotation resonances by an external potential. Astrophys. J. 233, 857-871.

Goldreich, P., Ward, W.R., 1973. The formation of planetesimals. Astrophys. J. 183, 1051-1062.

Goldreich, P., Murray, N., Longaretti, P.Y., Banfield, D., 1989. Neptune's story. Science $245,500-504$.

Goodman, J., 1993. A local instability of tidally distorted accretion disks. Astrophys. J. 406, 596-613.

Goodman, J., Pindor, B., 2000. Secular instability and planetesimal formation in the dust layer. Icarus $148,537-549$.

Goodman, J., Rafikov, R.R., 2001. Planetary torques as the viscosity of protoplanetary disks. Astrophys. J. 552, 793-802.

Greenberg, R., Davis, D.R., Hartmann, W.K., Chapman, C.R., 1977. Size distribution of particles in planetary rings. Icarus 30, 769-779.

Hahn, J.M., Ward, W.R., Rettig, T.W., 1995. Resonant trapping in a self-gravitating planetesimal disk. Icarus 117, 25-44.

Hayashi, C., Nakazawa, K., Adachi, I., 1977. Long-term behavior of planetesimals and the formation of the planets. Pub. Astr. Soc. Japan 20, 163-196

Hibbitts, C.A., Pappalardo, R., Klemaszewski, J., McCord, T.B., Hansen, G.B.2001. Comparing carbon dioxide distributions on Ganymede and Callisto. Proceedings of the 32nd LPSC, Houston 2001.

Hourigan, K., Ward, W.R., 1984. Radial migration of preplanetary material: implications for the accretion time scale problem. Icarus 60 , 29-39.

Ida, S., Bryden, G., Lin D.N.C., Tanaka, H. Orbital migration of Neptune and orbital distribution of trans-neptunian objects. Astrophys. J. 534 $428-445$.

Julian, W.H., Toomre, A., 1966. Non-axisymmetric responses of differentially rotating disks of stars. Astrophys. J. 146, 810-832.

Kary, D.M., Lissauer, J.J., Greenszweig, Y., 1993. Nebular gas drag and planetary accretion. Icarus 106, 288.

Klahr, H., Bodenheimer, P., 2001. Turbulence in accretion disks. The global baroclinic instability. Astronomische Gesellschaft Abstract Series, vol. 18, JENAM 2001, September 10-15, 2001, Munich, Germany.

Kley, W., 2000. On the migration of a system of protoplanets. Mon. Not. Roy. Astron. Soc. 313, L47-L51.

Kley, N., Papaloizou, J.C.B., Lin, D.N.C., 1993. On the angular momentum transport associated with convective eddies in accretion disks. Astrophys. J. 416, 679-688.

Korycansky, D.G., Papaloizou, J.C.B., 1996. A method for calculations of nonlinear shear flow: application to formation of giant planets in the solar nebula. Astrophys. J. Suppl. 105, 181-190.
Korycansky, D.G., Pollack, J.B., Bodenheimer, P., 1991. Numerical models of giant planet formation with rotation. Icarus 92, 234-251.

Kuramoto, K., Matsui, T., 1994. Formation of a hot proto-atmosphere on the accreting giant icy satellite: implications for the origin and evolution of Titan, Ganymede and Callisto. J. Geophys. Res. 99, 2118321200.

Lee, M.H., Peale, S.J., 2000. Making Hyperion. Presented at the 32nd DPS meeting, American Astronomical Society, October 2000.

Lewis, J.S., 1972. Low temperature condensation from the solar nebula. Icarus 16, 241-252.

Lewis, J.S., 1974. The temperature gradient in the solar nebula. Science 186, 440-443.

Li, H., Finn, J.M., Lovelace, R.V.E., Colgate, S.A., 2000. Rossby wave instability of thin accretion disks. II. Detailed linear theory. Astrophys. J. 533, 1023-1034.

Lin, D.N.C., 1981. Convective accretion disk model for the primitive solar nebula. Astrophys. J. 246, 972-984.

Lin, D.N.C., Papaloizou, J., 1980. On the structure and evolution of the primordial solar nebula. Mon. Not. Roy. Astron. Soc. 191, 37-48.

Lin, D.N.C., Papaloizou, J., 1993. On the tidal interaction between protostellar disks and companions, in: Levy, E.H., Lunine, J.I. (Eds.), Protostars and Planets III, Univ. of Arizona, Tucson, pp. 749-836.

Lissauer, J.J., 1995. Urey prize lecture: on the diversity of plausible planetary systems. Icarus 114, 217-236.

Lissauer, J.J., Stewart, G.R., 1993. Growth of planets from planetesimals, in: Levy, E.H., Lunine, J.I. (Eds.), Protostars and Planets III, Univ. of Arizona, Tucson, pp. 1061-1088.

Lissauer, J.J., Pollack, J.B., Wetherill, G.W., Stevenson, D.J., 1995, in: Matthews, M.S., Schumann, A.M. (Eds.), Formation of the Neptune System, Univ. Arizona Press, Tucson, p. 37.

Lovelace, R.V.E., Li, H., Colgate, S.A., Nelson, A.F., 1999. Rossby wave instability of keplerian accretion disks. Astrophys. J. 513, 805-810.

Lubow, S.H., 1981. Vertically driven resonances in accretion disks. Astrophys. J. 245, 274-285.

Lubow, S.H., Seibert, M., Artymowicz, P., 1999. Disk accretion onto high-mass planets. Astrophys. J. 526, 1001.

Lunine, J.I., 1989. Primitive bodies: molecular abundances in Comet Halley as probes of cometary formation environments, in: Weaver, H.A., Paresce, F., Danly, L. (Eds.), The Formation and Evolution of Planetary Systems, Cambridge Univ. Press, Cambridge, pp. 213-242.

Lunine, J.I., Stevenson, D.J., 1982. Formation of the Galilean satellites in a gaseous nebula. Icarus 52, 14-39.

Lunine, J.I., Tittemore, W.B., 1993. Origins of outer-planet satellites, in: Levy, E.H., Lunine, J.I. (Eds.), Protostars and Planets III, University of Arizona Press, Tucson, pp. 1149-1176.

Lynden-Bell, D., Pringle, J.E., 1974. The evolution of viscous disks and the origin of the nebula variables. Mon. Not. Roy. Astron. Soc. 168, 603-637.

Malhotra, R., 1993. Orbital resonances in the solar nebula: strengths and weaknesses. Icarus 106, 264-273.

Malhotra, R., Dermott, S.F., 1990. The role of secondary resonances in the orbital history of Miranda. Icarus 85, 444-480.

McKinnon, W.B., 1984. On the origin of Triton and Pluto. Nature 311, 544.

McKinnon, W.B., 1999. Midsize icy satellites, in: Kelly, J., Beatty, C., Peterson, C., Chaikin, A. (Eds.), The New Solar System, Sky Publishing Co., Cambridge, p. 297.

McKinnon, W.B., Simonelli, D.P., Schubert, G., 1997. Composition, internal structure, and thermal evolution of Pluto and Charon, in: Stern, S.A., Tholen, D.J. (Eds.), Pluto and Charon, The University of Arizona Press, Tucson, p. 295.

Melosh, H.J., 1989. Impact Cratering: A Geological Process. Oxford Univ. Press, New York.

Mizuno, H., 1980. Formation of the giant planets. Progress of Theoretical Physics 64, 544-557. 
Mizuno, H., Nakazawa, K., Hayashi, C., 1978. Instability of a gaseous envelope surrounding a planetary core and formation of giant planets. Progress of Theoretical Physics 60, 699-710.

Moore, J.M., Asphaug, E., Morrison, D., Spencer, J.R., Chapman, C.R., Bierhaus, B., Sullivan, R.J., Chuang, F.C., Klemaszewski, J.E., Greeley, R., Bender, K.C., Geissler, P.E., Helfenstein, P., Pilcher, C.B., 1999. Mass movement and landform degradation on the icy Galilean satellites: results of the Galileo nominal mission. Icarus 240, 294-312.

Mosqueira, I., Estrada, P.R. 2003. Formation of regular satellites of giant planets in an extended gaseous nebula II: satellite migration and survival. Icarus 163, 232-255.

Mosqueira, I., Estrada, P.R., Chambers, J.E., 2000. Satellitesimal feeding and the formation of the regular satellites. Proceedings of the 32nd DPS meeting.

Mousis, O., Gautier, D., Coustenis, A., 2001. The D/H ratio in methane in Titan: origin and history. Proceedings of the 33rd DPS meeting.

Mousis, O., Gautier, D., Bockelee-Morvan, D., 2002. An evolutionary turbulent model of Saturn's subnebula: implications for the origin of the atmosphere of Titan. Icarus 156, 162-175.

Orton, G., 1992. Ground based observations of Titan's thermal spectrum, in: Symposium on Titan. ESA SP-338, Noordwijk, Netherlands, pp. $81-85$.

Owen, T.C., Cruikshank, D.P., Dalle, O.C.M., Geballe, T.R., Roush, T.L., de Bergh, C., Bergh, T.L., Meier, R., Pendleton, Y.J., Khare, B.N., 2001. Decoding the domino: the dark side of Iapetus. Icarus 14, 160 172.

Podolak, M., Hubbard, W.B., Pollack, J.B., 1993. Gaseous accretion and the formation of the giant planets, in: Levy, E.H., Lunine, J.I. (Eds.), Protostars and Planets III, Univ. of Arizona, Tucson, pp. 1061-1088.

Pollack, J.B., Grossman, A.S., Moore, R., Graboske Jr., H.C., 1976. The formation of Saturn's satellites and rings as influenced by Saturn's contraction history. Icarus $29,35-48$.

Pollack, J.B., Grossman, A.S., Moore, R., Graboske Jr., H.C., 1977. A calculation of Saturn's gravitational contraction history. Icarus 30 , 111-128.

Pollack, J.B., Burns, J.A., Tauber, M.E., 1979. Gas drag in primordial circumplanetary envelopes: a mechanism for satellite capture. Icarus 37, 587-611.

Pollack, J.B., Lunine, J., Tittemore, W.C., 1991. Origin of the Uranian satellites, in: Bergstralh, J.T., Miner, E.D., Matthews, M.S. (Eds.), Uranus, Univ. of Arizona Press, Tucson.

Pollack, J.B., Hollenbach, D., Beckwith, S., Simonelli, D.P., Roush, T., Fong, W., 1994. Composition and radiative properties of grains in molecular clouds and accretion disks. Astrophys. J. 421, 615639.

Pollack, J.B., Hubickyj, O., Bodenheimer, P., Lissauer, J.J., Podolak, M., Greenzweig, Y., 1996. Formation of the giant planets by concurrent accretion of solids and gas. Icarus 124, 62-85.

Prinn, R.G., Fegley Jr., B., 1981. Kinetic inhibition of CO and $\mathrm{N}_{2}$ reduction in circumplanetary nebulae: implications for satellite composition. Astrophys. J. 249, 308-317.

Prinn, R.G., Fegley Jr., B., 1989. Solar nebula chemistry: origin of planetary, satellite, and cometary volatiles, in: Atreya, S.K., Pollack, J.B., Matthews, M.S. (Eds.), Origin and Evolution of Planetary and Satellite Atmospheres, Univ. of Arizona Press, Tucson, pp. 78-136.
Quataert, E., Chiang, E.I., 2000. Angular momentum transport in particle and fluid disks. Astrophys. J. 543, 432-437.

Quillen, A.C., Trilling, D.E., 1998. Do proto-jovian planets drive outflows? Astrophys. J. 508, 707-713.

Ruden, S.P., Lin, D.N.C., 1986. The global evolution of the primordial solar nebula. Astrophys. J. 308, 883-901.

Ryu, D., Goodman, J., 1992. Convective instability in differentially rotating disks. Astrophys. J. 388, 438-450.

Safronov, V.S., 1969. Evolution of the Protoplanetary Cloud and Formation of the Earth and Planets. Nauka, Moscow. [Transl.: Israel Program for Scientific Translations, 1972. NASA TTF-677].

Saha, P., Tremaine, S., 1993. The orbits of the retrograde jovian satellites. Icarus 106, 549.

Schubert, G., Stevenson, D.J., Ellsworth, K., 1981. Internal structures of the Galilean satellites. Icarus 47, 46-59.

Shu, F.H., Johnstone, D., Hollenbach, D., 1993. Photoevaporation of the solar nebula and the formation of the giant planets. Icarus 106, 92-101.

Squyres, S.W., Sagan, C., 1983. Albedo asymmetry of Iapetus. Nature 303, 782-785.

Stevenson, D.J., 2001. Jupiter and its Moons. Science 294, 71-72.

Stevenson, D.J., Harris, A.W., Lunine, J.I., 1986. Origins of satellites, in: Burns, J.A., Matthews, M.S. (Eds.), Satellites, Univ. of Arizona Press, Tucson.

Stone, J.M., Balbus, S.A., 1996. Angular momentum transport in accretion disks via convection. Astrophys. J. 464, 364-372.

Supulver, K.D., Lin, D.N.C., 2000. Formation of icy planetesimals in a turbulent solar nebula. Icarus 146, 525-540.

Suttner, G., Yorke, H.W., 2001. Early dust evolution in protostellar accretion disks. Astrophys. J. 551, 461-477.

Takeuchi, T., Miyama, S.M., Lin, D.N.C., 1996. Gap formation in protoplanetary disks. Astrophys. J. 460, 832.

Ward, W.R., 1993. Density waves in the solar nebula: planetesimal velocities. Icarus 106, 274-287.

Ward, W.R., 1997. Protoplanet migration by nebula tides. Icarus 126 , 261-281.

Weidenschilling, S., 1974. A model for accretion of the terrestrial planets. Icarus 22, 426-435.

Weidenschilling, S., 1984. Evolution of grains in a turbulent solar nebula. Icarus $60,553-567$

Weidenschilling, S., 1988. Formation processes and timescales for meteorite parent bodies, in: Kerridge, J.F., Matthews, M.S. (Eds.), Meteorites and the Early Solar System, Univ. of Arizona Press, Tucson.

Weidenschilling, S., 1997. The origin of comets in the solar nebula: a unified model. Icarus 127, 290-306.

Weidenschilling, S., Cuzzi, J.N., 1993. Planetesimal formation in the protoplanetary nebula, in: Gehrels, T. (Ed.), Protostars and Planets, Univ. of Arizona Press, Tucson.

Wetherill, G.W., 1980. Formation of the terrestrial planets. Ann. Rev. Astron. Astrophys. 18, 77-113.

Wisdom, J., Peale, S.J., Mignard, F., 1984. The chaotic rotation of Hyperion. Icarus 58, 137-146.

Wuchterl, G., Guillot., T., Lissauer, J.J., 2000. In: Mannings, V., Boss, A., Russel, S. (Eds.), Protostars and Planets IV, Univ. of Arizona Press, Tucson, AZ, 2000, pp. 1081-1109. 